\title{
VISUALIZING FLOW PATTERNS IN COUPLED GEOMECHANICAL SIMULATION USING STREAMLINES
}

\author{
A Thesis \\ by \\ PRANNAY PARIHAR \\ Submitted to the Office of Graduate Studies Of \\ Texas A\&M University \\ in partial fulfillment of the requirements for the degree of \\ MASTER OF SCIENCE
}

December 2008

Major Subject: Petroleum Engineering 


\title{
VISUALIZING FLOW PATTERNS IN COUPLED GEOMECHANICAL SIMULATION USING STREAMLINES
}

\author{
A Thesis \\ by \\ PRANNAY PARIHAR \\ Submitted to the Office of Graduate Studies Of \\ Texas A\&M University \\ in partial fulfillment of the requirements for the degree of \\ MASTER OF SCIENCE
}

Approved by:

Chair of Committee, Akhil Datta-Gupta

Committee Members, Yalchin Efendiev

Daulat D. Mamora

Head of Department, Stephen A. Holditch

December 2008

Major Subject: Petroleum Engineering 


\author{
ABSTRACT \\ Visualizing Flow Patterns in Coupled Geomechanical Simulation Using Streamlines. \\ (December 2008) \\ Prannay Parihar, B.S., Indian School of Mines, Dhanbad, India \\ Chair of Advisory Committee: Dr. Akhil Datta-Gupta
}

Reservoir geomechanics is a production induced phenomena that is experienced in large number of fields around the world. Hydrocarbon production changes the pore pressure which in turn alters the in-situ stress state. For reservoirs that are either stress sensitive or where rock is soft and unconsolidated, stresses have appreciable effect on rock properties like porosity and permeability. Anisotropic and isotropic permeability changes affect flow direction and movement of flood front thereby influencing well performance and reservoir productivity. Coupling of geomechanical calculation with multi-phase flow calculation is needed to make prudent predictions about the reservoir production and recovery. The post processing tools provided with the simulators cannot monitor flood front movement and fail to capture important information like flow directionality and dominant phase in a flow. Geomechanical simulation is combined with streamline tracing to aid in better understanding of the reservoir dynamics through visualization of flow patterns in the reservoir. Streamline tracing is a proved reservoir engineering tool that is widely used by industry experts to capture information on flood movement, injector-producer relations and swept area. 
In the present research, we have incorporated total velocity streamlines and phase streamlines for coupled geomechanical simulation and compared the results with streamline tracing for conventional reservoir simulator to explain geomechanics behavior on reservoir flow processes in a more detailed and appealing manner. Industry standard simulators are used for coupled geomechanical simulation and conventional simulation and streamline tracing has been done through in-house tracing code.

The research demonstrates the benefits and power of streamline tracing in visualizing flow patterns through work on two cases; first, a synthetic case for studying water injection in a five spot pattern and second, a SPE $9^{\text {th }}$ comparative study. The research gives encouraging results by showing how geomechanics influences reservoir flow paths and reservoir dynamics through visualization of flow. The streamlines captures flow directionality, information regarding appearance and disappearance of gas phase and the connectivity between injector and producer. 


\section{DEDICATION}

I would like to dedicate this thesis to my beloved parents in India for their love, care and constant support. 


\section{ACKNOWLEDGMENTS}

I would like to express my deepest gratitude and appreciation to my advisor, Dr. Akhil Datta-Gupta for his valuable guidance and financial support. I would also like to express my sincere thanks to Dr. Daulat Mamora and Dr Yalchin Efendiev for serving as committee members.

I want to thank Baljit Sebi in Schlumberger for providing help in dealing with issues related to the ECLIPSE simulator. I would also like to give my special thanks to Sarwesh Kumar for his constant encouragement and valuable advice for the project.

Finally, I want to thank Eduardo Jimenez and Adedayo Oyerinde for their help and support. 


\section{TABLE OF CONTENTS}

Page

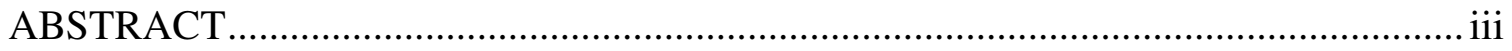

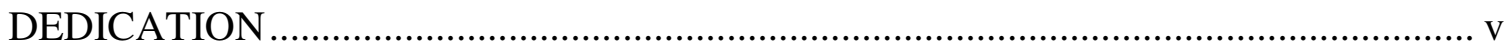

ACKNOWLEDGMENTS ............................................................................... vi

TABLE OF CONTENTS .................................................................................. vii

LIST OF FIGURES .........................................................

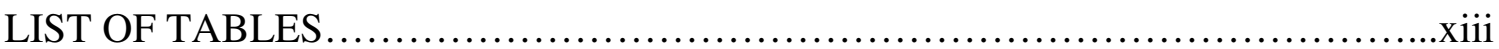

\section{CHAPTER}

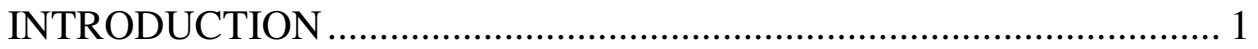

1.1 Literature Review: Coupled Reservoir Simulation................... 3

1.2 Literature Review: Streamline Simulation .............................. 4

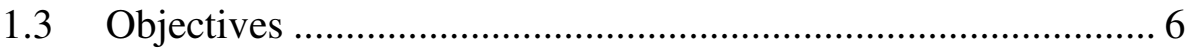

II RESERVOIR GEOMECHANICS ................................................. 8

2.1 Modeling Approach for Deformable Porous Media .................. 8

2.1.1 Fluid Flow Theory: Basic Relations ............................... 9

2.1.2 Governing Equations ................................................ 10

2.1.3 Linear Poroelastic Theory: Basic Relations................... 14

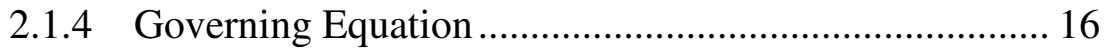

2.1.5 Effective Stress Concept ........................................... 17

2.2 Nondeformable Porous Media ............................................. 18

2.3 Type of Coupling .................................................................. 19

2.3.1 Full Coupling .......................................................... 20

2.3.2 One-way Coupling/ Explicit Coupling ........................ 20

2.3.3 Loose Coupling/Iterative Coupling.............................. 21

2.3.3.1 Loose Coupling Algorithm .......................... 21

2.4 Modifications for Simulation..............................23 
2.4.1 Modifications to Fluid Flow: Porosity Updates............. 23

2.4.2 Modifications to Fluid Flow: Permeability Updates....... 24

2.4.3 Modifications to Geomechanics: Porosity Updates ........ 25

2.4.4 Modifications to Geomechanics: Permeability Updates. 26

2.5 Description of Reservoir Simulator ......................................... 27

2.5.1 Compositional Flow Simulation ................................. 27

2.5.1.1 Flash Calculation ......................................... 28

2.5.1.2 Flux Calculation............................................ 28

2.5.2 Partly Coupled Geomechanical Simulation ................... 29

2.5.2 .1 Elastic Stress Equations ............................... 30

2.5.2 .2 Variables and Solution of the Equations.......... 32

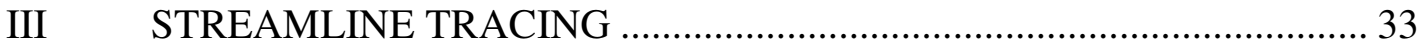

3.1 Pollock's Tracing Algorithm for Rectangular Cells .................. 33

3.2 Extension of Pollock's Algorithm to Corner Point Grid ............ 36

3.2.1 Pseudo Time of Flight............................................... 40

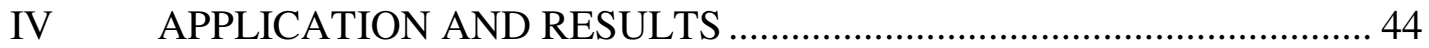

4.1 Synthetic Case Study ........................................................ 44

4.1.1 Geomechanics Impact on Flow Direction.................... 51

4.1.2 Geomechanics Impact on Permeability ....................... 55

4.1.3 Geomechanics Impact on Water Production................. 65

4.2 SPE $9^{\text {th }}$ Comparative Study............................................... 72

4.2.1 Comparison of Production for Producer19 .................. 80

4.2.2 Comparison of Production for Producer 4..............84

4.2.3 Water Injection Rate Comparison.....................91

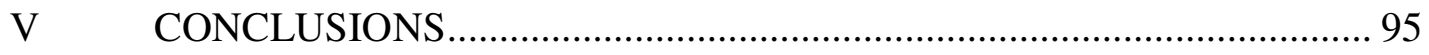

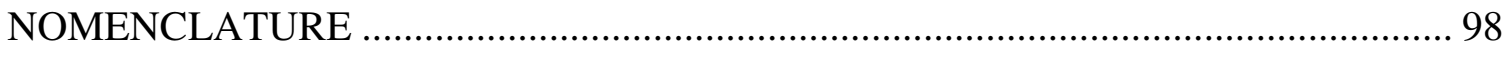

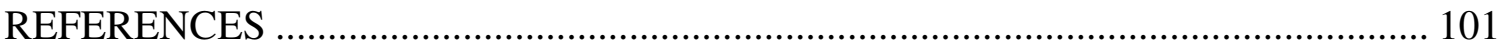

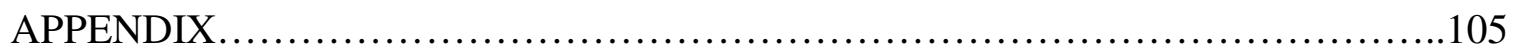

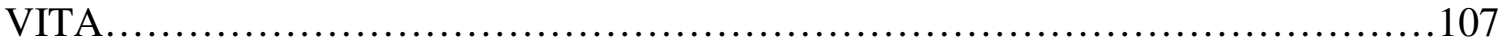




\section{LIST OF FIGURES}

Page

Figure 1 - Schematic of Unjacketed and Jacketed Compressibility Test ${ }^{19}$ 12

Figure 2 - Flow Chart for Lose Coupling Algorithm ${ }^{32}$ 23

Figure 3 - Grid Block Time of Flight Calculation ${ }^{16}$ 34

Figure 4 - Corner Point Cell Described by Eight Corners ${ }^{16}$ 37

Figure 5 - Isoparametric Transformation ${ }^{16}$ 38

Figure 6 - Schematic Showing the Pseudo Time of Flight in a Unit Cube ${ }^{16}$ 40

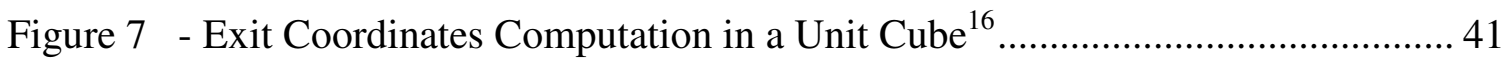

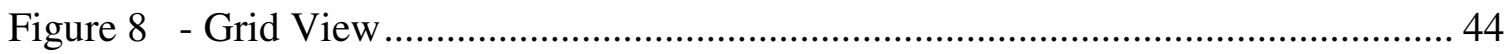

Figure 9 - Top View of Permeability Distribution for the First Reservoir Layer .......... 45

Figure 10 - Field Pressure for Runs With and Without Geomechanics Option................ 47

Figure 11 - Plots Showing Oil Production Rate for All the Producers ........................... 48

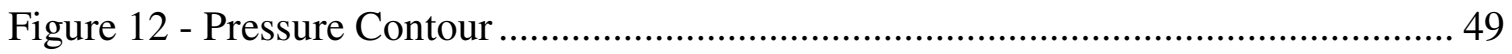

Figure 13 - Time of Flight Profile for Total Velocity Tracing at $1^{\text {st }}$ Report Step........... 49

Figure 14 - Time of Flight Profile for Total Velocity Tracing at $2^{\text {nd }}$ Report Step........... 50

Figure 15 - Time of Flight Profile for Total Velocity Tracing at $3^{\text {rd }}$ Report Step ........... 51

Figure 16 - Time of Flight Profile for Total Velocity Tracing at $4^{\text {th }}$ Report Step ........... 52

Figure 17 - Time of Flight Profile for Total Velocity Tracing at $7^{\text {th }}$ Report Step ........... 52

Figure 18 - Time of Flight Profile for Total Velocity Tracing at $11^{\text {th }}$ Report Step ......... 53

Figure 19 - Plot Showing Field Oil Production Rate for Runs With and Without Geomechanics Option 
Figure 20 - Oil Saturation Contours at $3^{\text {rd }}$ Report Step .............................................. 54

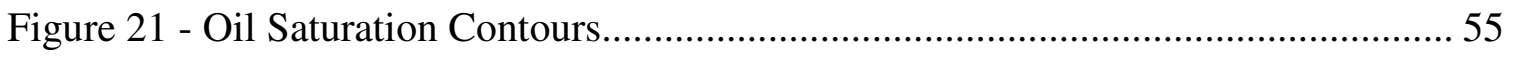

Figure 22 - Contour for Permeability in X-direction for Top Layer.................................. 56

Figure 23 - Time of Flight Profile for Water Phase Tracing ………………………...... 57

Figure 24 - Pressure Contours................................................................................ 58

Figure 25 - Pressure Contours at Last Report Step ............................................................ 59

Figure 26 - Effective Stress and Rock Displacement in Z Direction................................. 60

Figure 27 - Effective Stress and Rock Displacement in Y Direction .............................. 61

Figure 28 - Effective Stress and Rock Displacement in X Direction ............................... 62

Figure 29 - Principal Stress 3 and Permeability Modifier for Geomechanics Run .......... 63

Figure 30 - Principal Stress 3 and Permeability Modifier for Geomechanics Run at Last

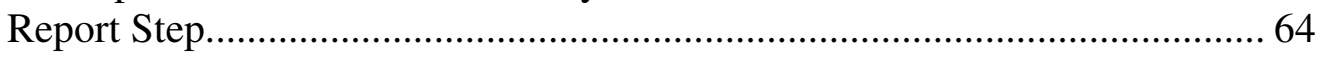

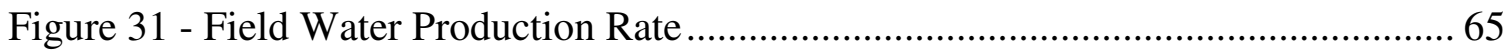

Figure 32 - Plots Showing Water Production Rate for All the Producers ......................... 66

Figure 33 - Plots Showing Water Cut for All the Producers ........................................... 67

Figure 34 - Time of Flight Profile for Water Phase Tracing …….................................... 68

Figure 35 - Time of Flight Profile for Water Phase Tracing at Different Report Steps ... 69

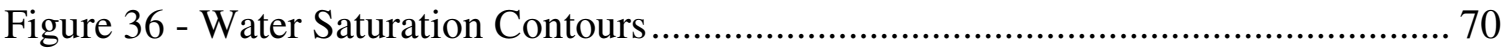

Figure 37 - Water Saturation Contours at Last Report Step …………………………... 71

Figure 38 - 3D View of Modified Grid for Geomechanics Study .................................... 72

Figure 39 - Top View of Permeability Distribution for the First Reservoir ..................... 72

Figure 40 - Cross-Sectional View of Grids................................................................. 73

Figure 41 - Fluid Contacts After Initialization of Study ................................................. 73 
Figure 42 - Comparison of Pressure and Oil production for Runs With Geomechanics Option and Without Geomechanics Option 74

Figure 43 - Side View of the Streamline Tracing .................................................... 76

Figure 44 - Total Velocity Tracing and Individual Phase Tracing for $1^{\text {st }}$ Report Step.... 77

Figure 45 - Total Velocity Tracing for Last Report Step......................................... 78

Figure 46 - Individual Phase Tracing for Last Report Step ...................................... 79

Figure 47 - Gas Production Rate and Water Production Rate for Producer 19.............. 80

Figure 48 - Time of Flight for Gas Phase Tracing at Last Report Step - Top View ....... 81

Figure 49 - Time of Flight for Gas Phase Tracing at Last Report Step - Side View ....... 81

Figure 50 - Time of Flight for Total Velocity Tracing at Last Report Step - Top View . 82

Figure 51 - Time of Flight for Total Velocity Tracing at $3^{\text {rd }}$ Report Step - Top View .... 83

Figure 52 - Time of Flight for Total Velocity Tracing at $3^{\text {rd }}$ Report Step - Side View ... 83

Figure 53 - Gas Production Rate and Water Production Rate for Producer 4 ................ 84

Figure 54 - Time of Flight for Gas Phase Tracing at $5^{\text {th }}$ Report Step - Top View.......... 85

Figure 55 - Time of Flight for Gas Phase Tracing at Last Report Step - Top View....... 85

Figure 56 - Time of Flight for Gas Phase Tracing at Last Report Step - Side View ....... 86

Figure 57 - Gas Phase Pressure at Last Report Step................................................ 87

Figure 58 - Time of Flight for Water Phase Tracing at $4^{\text {th }}$ Report Step - Top View ....... 88

Figure 59 - Time of Flight for Water Phase Tracing at $4^{\text {th }}$ Report Step - Side View ...... 88

Figure 60 - Permeability Distribution for All the Layers - Side View.......................... 89

Figure 61 - Water Phase Pressure at $4^{\text {th }}$ Report Step for All the Layers - Side View ...... 90

Figure 62 - Water Phase Pressure at $4^{\text {th }}$ Report Step for First Layer............................. 90

Figure 63 - Water Injection Rate for Injector ................................................... 91 
Figure 64 - Water Phase Tracing for Injector at $1^{\text {st }}$ Report Step ................................ 92

Figure 65 - Time of Flight for Water Phase Tracing at $1^{\text {st }}$ Report Step - Side View ...... 92

Figure 66 - Reservoir Pressure at $1^{\text {st }}$ Report Step - Top View .................................... 93

Figure 67 - Water Phase Pressure at $1^{\text {st }}$ Report Step for Last Layer.............................. 94

Figure 68 - Water Phase Pressure at $1^{\text {st }}$ Report Step for First Layer ............................. 94 
xiii

\section{LIST OF TABLES}

TABLE $\quad$ Page

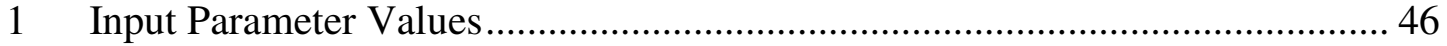

2 Principal Stress vs Permeability Multiplier .............................................. 46 


\section{CHAPTER I}

\section{INTRODUCTION}

A fluid saturated porous media is characterized by its porosity, permeability as well as the properties of its constituents like solid matrix and fluids. The porous media is surrounded by geological formations that exert overburden and side-burden stresses. Because of these in-situ stresses and pressure exerted by fluids, the reservoir rock is always under tensile/compressive stress state. Reservoir Geomechanics occur in many oil fields around the world and is responsible for providing additional hydrocarbon recovery through compaction drive as well as important consequences like subsidence. Production of oil and gas decreases the pore pressure and increases effective overburden stress on reservoir rock which in turn causes rock compaction. Similarly, fluid injection increases pore pressure and decreases effective overburden load which in turn causes reservoir to dilate. These changes of the pore pressure and the stress state of the reservoir that gives rise to a change in volume of both reservoir fluids and reservoir rocks affects reservoir producibility.

This thesis follows the style of Society of Petroleum Engineers Journal. 
Fluid composition and pore pressure control volumetric behavior of reservoir fluids whereas the volumetric response of the reservoir rock depends on the mechanical properties of the rock material and the combined effect of changes in pore pressure and stress state. Conventional porous fluid-flow modeling treats permeability and rock compressibility to be stress independent and thus do not account for the interactions between fluid flow and rock whereas coupled fluid flow and geomechanical modeling incorporates the effect of stress dependent permeability and rock mechanical properties on reservoir productivity. So, for the simulation study of stress sensitive reservoirs where the volumetric deformation of the rock has appreciable effects on both porosity and permeability, it has become imperative to go for coupled geomechanical simulators.

In the present research, in addition to coupled geomechanical and fluid flow simulations, we will be utilizing streamlines as a tool to aid in understanding flow behavior and reservoir processes. This will be accomplished by tracing and comparing streamlines for both, coupled geomechanical simulation and conventional reservoir simulation. Streamlines provide a natural means of dynamic reservoir characterization by delineating fast and slow flow paths. Also, the evolution of flood fronts and their interaction with heterogeneity can be visualized using streamline models. These advantages of streamline will have significant influence in understanding impact of geomechanics and stress dependent permeability on reservoir dynamics. 


\subsection{Literature Review: Coupled Reservoir Simulation}

Geomechanics analysis has shown to increase the overall value to various development projects. A comprehensive geomechanics study was carried out on a deepwater Gulf of Mexico field by Chhajlani et al. ${ }^{5}$. The study addressed issues related to optimizing field development with respect to critical drilling, completion and reservoir issues. The log-based geomechanics models used to evaluate sand production and pressure dependent pore volume compressibility have aided in reducing project risks and increasing project life.

In-situ stress depletion is one of the main reasons of productivity reduction in low permeability reservoirs. A field study was carried out for stress-sensitive permeability in near critical Cupiagua gas condensate reservoir in Colombia by Giraldo et al. ${ }^{6}$ Presence of natural fractures and unusual tectonic regime make it an interesting case to study impact of geomechanics on reservoir performance. Results from the study show that permeability reduction is due to condensate effects and in-situ stress depletion.

Input parameters that go into the geomechanical modeling also play an important role in reservoir behavior due to fluid production. Kristiansen ${ }^{7}$ did a study to improve the geomechanical input to the full field compaction/subsidence prediction model used to plan long extended reach wells in the Valhall field. The results are also applicable for other geomechanical activities in the field like well design, water injection, hydraulic fracturing and design of waterflood.

Generic studies, involving coupled geomechanical, thermal and fluid modeling have elicited the concept that preferred directionality in oilfield waterflooding is stress- 
related and progressive with time. Heffer et $a l .{ }^{8}$ did coupled geomechanical, thermal and fluid flow modeling as an aid to improve waterflood sweep efficiency. For hydrocarbon reservoirs with stress dependent permeability, the coupled interaction between the geomechanics and fluid production may significantly influence both stress state and fluid flow in the reservoir. To understand this, a fully coupled geomechanics and fluid-flow model was developed to analyze pressure-transient problems in stress-sensitive reservoirs by Chin et al. ${ }^{9}$

Settari et $a l .{ }^{33}$ have described a method for modular coupling of a commercial reservoir simulator with a three-dimensional (3D) stress code and fracture propagation model. They demonstrated the utility of the method on several examples and the comparison with an uncoupled solution shows that significantly different and more realistic answers are obtained with the coupled modeling.

\subsection{Literature Review: Streamline Simulation}

Streamline simulators are gaining increasing acceptance because of their computational advantage, intuitive appeal and ability to visualize the flow patterns in 3D. Streamline models provide us with a tool for fast flow simulation and rapid screening and ranking of 3D models. Streamline models have variety of applications like rate allocation and flood-front management, integration of water-cut and tracer data into reservoir description, upgridding from fine-scale models and visualization of interactions between flood front and heterogeneity. 
Giordano et al. ${ }^{10}$ were successful in developing a dynamic model for the Lekhwair field by integrating streamlines with well performance data and novel diagnostic plots. The integrated reservoir management workflow provides efficient ways for maximizing oil recovery in a mature field. Baker et $a l^{11}$. in their work of full-field modeling with streamline simulation, have credited streamlines as the primary reason for faster history matching due to a number of advantages that they offer. In their case studies, streamlines clearly identified which producer-injector pairs communicate strongly through flow visualization. Streamlines reduced the uncertainty associated with outer-boundary conditions by allowing use of large number of wells and streamline flow paths indicated that idealized drainage patterns do not exist in real fields. In addition to this, streamlines also provided new flow information like well-connectivity, drainage volumes and well allocation factors which cannot be derived from conventional simulation methods.

Characterizing fluid flow in fractured reservoir is difficult because of heterogeneity and complex intersection patterns of fractures. Park et $a l^{12}$. investigated interwell connectivity and tracer transport in 3D fractured reservoir using streamline simulation to show improved results and to overcome limitations posed by complexity and uncertainty of fractures. Streamlines have proved to be an ideal reservoir management tool for fields with mature waterflood. Naguib et $a l^{13}$. used streamline simulation for managing a heavy-oil waterflood in South Oman and the results show the manner in which streamline simulation aids in reservoir management by identifying unswept reserves, quick evaluation of multiple forecast scenarios and information like well-pair interactions. 
The concept of time of flight along a streamline was introduced by Datta-Gupta \& King $^{18}$. Most of the current streamline based flow simulators use this concept because it facilitates splitting of the 3D problem into 1D problems and makes flow simulations much faster. The time of flight concept has been the most significant contribution in streamline simulation and it will be used very extensively in the present research.

Streamlines as a reservoir engineering tool has lot of potential for management of stress sensitive reservoirs where geomechanics play dominant role in the flow dynamics of reservoir. To the best knowledge of the author, such a step of combining streamline tracing with geomechanical simulation has never been attempted and with our present research study we try to accomplish that.

\subsection{Objectives}

The main goal of the present research is to trace total velocity streamlines and phase streamlines for coupled geomechanical simulation and conventional reservoir simulation and compare the results. Simulation study will be performed on two cases; a synthetic case and a SPE $9^{\text {th }}$ comparative study. Comparison of the results from the two simulators will be done for each case in terms of the following parameters:

- Streamline trajectories and flow path

- Streamline time of flight

- Movement of flood front

- Flow directionality in the reservoir

- Pressure and well flow rates 
- Recovery efficiency

The comparison will lead to a better understanding of reservoir flow processes and reservoir production performance. In-house streamline tracing code TAMU DESTINY will be used to trace streamlines from the output of the ECLIPSE $300 \AA$ simulator and results will be visualized in FLOVIZ®.

Another area of research will be to study affect of stress-dependent permeability on the reservoir dynamics and fluid flow directionality. Hydrocarbon production affects pore pressure which in turn alters the existing in-situ stresses in the rock. By including table for permeability multiplier versus principal stress values, the effect of stresses on streamline profiles will be studied. 


\section{CHAPTER II}

\section{RESERVOIR GEOMECHANICS}

The fluid flow processes in hydrocarbon production involve interactions between multiphase flow and stress/strain behavior in porous media that results in reservoir compaction during production and subsequent subsidence and wellbore stability. Due to complexity of the equations involved and the cost of coupled modeling in terms of simulation time, geomechanics is approximated in conventional reservoir simulators by a pressure dependent treatment of porosity (or rock compressibility) and permeability. However, such approximations are unacceptable in situations where there is strong coupling for example, in case of reservoir modeling of unconsolidated porous media where the changes of porosity and permeability from stress changes and failure of soil cannot be represented by simply rock compressibility. Coupled geomechanical reservoir modeling correctly represents the dependence of porosity and permeability on effective stress and deformation of the porous media.

\subsection{Modeling Approach for Deformable Porous Media}

Equations governing isothermal, single-phase fluid flow in a deformable porous media are highlighted in this section to compare it with equations governing isothermal, single-phase fluid flow for non-deformable porous media. Generalization to multiphase

flow follows the same overall approach through an extension of the Darcy's law to multiphase flow by including individual phase velocities and fractional flow. 


\subsubsection{Fluid Flow Theory: Basic Relations}

Fluid flow in porous media is characterized by three basic principles:

- Mass conservation:

$$
\begin{array}{ll}
\text { Fluid: } & \nabla \bullet(\rho \phi v)+\frac{\partial(\phi \rho)}{\partial t}=0 \ldots \ldots \ldots \ldots \ldots \ldots . . . \\
\text { Solid: } \quad \nabla \bullet\left[\rho_{s}(1-\phi) v_{s}\right]+\frac{\partial\left[(1-\phi) \rho_{s}\right]}{\partial t}=0 .
\end{array}
$$

- Darcy's Law:

$$
\phi\left(v-v_{s}\right)=-\frac{k}{\mu} \nabla p
$$

- Equation of State:

$$
c=\frac{1}{\rho} \frac{\partial \rho}{\partial p}
$$

For a deformable porous media, Darcy's law is expressed as fluid velocity relative to the moving solid as shown by Eq.2.3 (due to Gersevanov in 1934 as cited by Biot ${ }^{20}$ and Verrujit ${ }^{31}$ ). In the mass conservation equation for fluid (Eq.2.1), $\phi v$ is fluid bulk volumetric flux and $(1-\phi) v_{s}$ is the solid bulk volumetric flux in mass conservation equation for solid (Eq.2.2).

Rock properties are a function of mean normal stress and fluid pressure whereas fluid density and viscosity are assumed to be a function of fluid pressure only. 


\subsubsection{Governing Equations}

Substituting Darcy's law into mass conservation equation (Eq.2.1) gives:

$$
\nabla \bullet\left(\rho \frac{k}{\mu} \nabla p\right)=\frac{\partial(\phi \rho)}{\partial t}+v_{s} . \nabla(\phi \rho)+\phi \rho \nabla \cdot v_{s}
$$

or

$$
\nabla \bullet\left(\rho \frac{k}{\mu} \nabla p\right)=\frac{d(\phi \rho)}{d t}+\phi \rho \nabla \cdot v_{s}
$$

where $\frac{d(.)}{d t}$ is the material derivative with respect to a moving solid defined as:

$$
\frac{d(.)}{d t}=\frac{\partial(.)}{\partial t}+v_{s} . \nabla(.)
$$

Eq.2.7 links the material derivative which is a Langrangian concept, to a Eulerian or spatial description. Expanding Eq.2.6, we get:

$$
\nabla \bullet\left(\rho \frac{k}{\mu} \nabla p\right)=\phi \rho\left(\frac{1}{\rho} \frac{d \rho}{d t}+\frac{1}{\phi} \frac{d \phi}{d t}+\phi \rho \nabla \bullet v_{s}\right)
$$

Expanding first term in Eq.2.2 and applying Eq.2.7 we get:

$$
\nabla \bullet v_{s}=\frac{1}{(1-\phi) \rho_{s}} \frac{d\left[(1-\phi) \rho_{s}\right]}{d t}
$$

Now $\phi=V_{p} / V_{b}$ and $V_{b}=V_{p}+V_{s}$, so Eq.2.9 is equivalent to

$$
\nabla \bullet v_{s}=\frac{1}{V_{b}} \frac{d V_{b}}{d t}
$$

Eq.2.10 is the divergence of solid velocity which is rate of change of bulk volume. 
Applying the relation $d \phi / \phi=d V_{p} / V_{p}-d V_{b} / V_{b}$ and Eq.2.10 to Eq.2.8:

$$
\nabla \bullet\left(\rho \frac{k}{\mu} \nabla p\right)=\phi \rho\left(\frac{1}{\rho} \frac{d \rho}{d t}+\frac{1}{V_{p}} \frac{d V_{p}}{d t}\right)
$$

Right hand side of Eq.2.11 represents rate of change of fluid density and pore volume. It is the fundamental equation for coupled fluid flow analysis. Different interpretations of the pore volume change due to different boundary conditions results in different governing equations and associated total compressibility. A proper interpretation of pore volume change is necessary for achieving fluid flow and geomechanics coupling.

The fluid compressibility is related to change in fluid density as:

$c \frac{\partial p}{\partial t}=\frac{1}{\rho} \frac{\partial \rho}{\partial t} ; \quad c \nabla p=\frac{1}{\rho} \nabla \rho ; \quad c \frac{d p}{d t}=\frac{1}{\rho} \frac{d \rho}{d t}$

Following Geertsma ${ }^{27}$, and Brown and Korringa ${ }^{28}, d V_{p} / V_{p}$ in Eq.2.11 can be written as:

$-\frac{d V_{p}}{V_{p}}=\frac{1}{V_{p}}\left(\frac{\partial V_{p}}{\partial p_{d}}\right)_{p} d p_{d}-\frac{1}{V_{p}}\left(\frac{\partial V_{p}}{\partial p}\right)_{p_{d}} d p$

here, $p_{d}$ is the differential pressure, $p_{d}=p_{c}-p$, where $p_{c}$ and $p$ are confining pressure and fluid pressure. The second term in right hand side in Eq.2.13 is assumed to be the unjacketed bulk compressibility $c_{s}\left(=1 / \mathrm{K}_{\mathrm{s}}\right)$ measured by allowing the fluid to penetrate the connected pores such that fluid pressure acts fully on solid. Under such conditions, $d p_{c}=d p$ and $d p_{d}=0$ or $p_{d}$ is constant.

Through the reciprocal theorem of elasticity, Geertsma ${ }^{27}$ showed that first partial derivative in Eq.2.13 can be expressed in terms of the unjacketed bulk compressibility $c_{s}$ 
and drained jacketed bulk compressibility $c_{b}\left(=1 / K_{b}\right)$. A drained condition means constant pore-pressure during the test.

The change of pore volume in Eq.2.13 can be expressed in terms of porosity and two measurable compressibilities, $c_{s}$ and $c_{b}$ as:

$$
\begin{gathered}
\phi \frac{d V_{p}}{V_{p}}=\frac{d V_{p}}{V_{b}}=-\left(c_{b}-c_{s}\right) d p_{d}-\phi c_{s} d p \\
=\left[c_{b}-(1+\phi) c_{s}\right] d p+\left(c_{b}-c_{s}\right) d \sigma_{m}
\end{gathered}
$$

where $\sigma_{m}$ is the mean normal stress which is equal to negative of confining pressure, i.e.,

$\sigma_{m}=-p_{c}$ and also $d p_{d}=d p_{c}-d p$

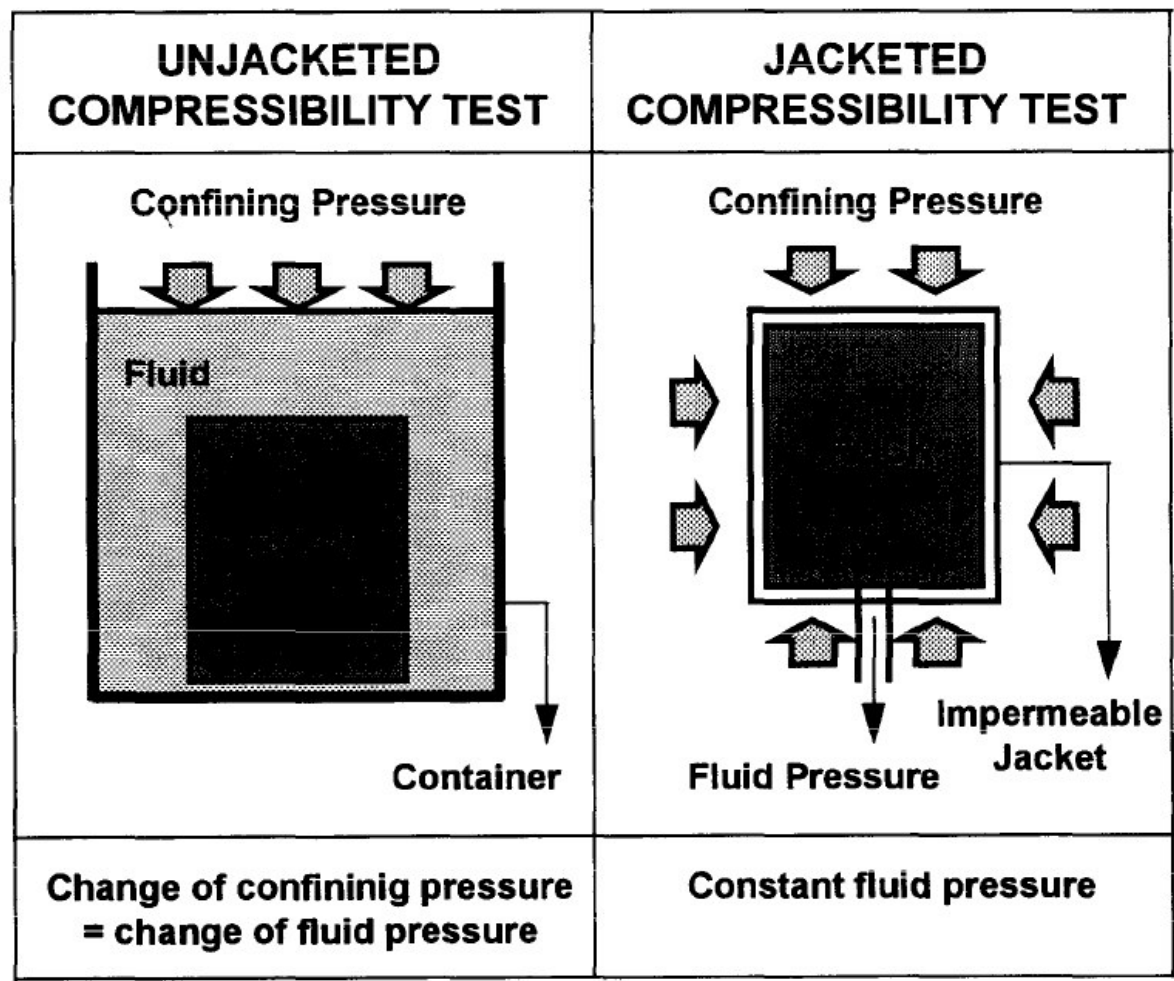

Figure 1 - Schematic of Unjacketed and Jacketed Compressibility Test ${ }^{19}$ 
Substituting eq.2.12 and 2.14 into Eq.2.11 results in:

$$
\nabla \bullet\left(\frac{k}{\mu} \nabla \rho\right)=\left[\phi c+c_{b}-(1+\phi) c_{s}+\left(c_{b}-c_{s}\right) \frac{d \sigma_{m}}{d p}\right] \frac{d \rho}{d t}
$$

or

$$
\nabla \cdot\left(\frac{k}{\mu} \nabla p\right)+\frac{k}{\mu} c(\nabla p)^{2}=\left[\phi c+c_{b}-(1+\phi) c_{s}+\left(c_{b}-c_{s}\right) \frac{d \sigma_{m}}{d p}\right] \frac{d p}{d t}
$$

For Eq.2.15, primary variable is fluid density and for Eq.2.16, primary is fluid pressure. There are two major features of Eq.2.15 and Eq.2.16; first, the material derivative $d(.) / d t$ is due to consideration of deformation $\left(v_{s} \neq 0\right)$ and second, the term $d \sigma_{m} / d p$ is due to interpretation of pore volume change. Using fluid density as primary variable leads to problems because of nonlinear terms in the coupled mode. So, Eq.2.15 and Eq.2.16 will be simplified using fluid pressure as primary variable.

The following two assumptions are imposed for eq.2.15:

- The fluid is slightly compressible

$$
\rho=\rho_{o} e^{c\left(p-p_{o}\right)} \cong \rho_{o}\left[1+c\left(p-p_{o}\right)\right]
$$

- The material derivative can be approximated by partial derivative

$$
\frac{d(.)}{d t}=\frac{\partial(.)}{\partial t}
$$

This means that the dot product in Eq.2.7 is neglected, $v_{s} . \nabla()<.<\partial(.) / \partial t$. Physical interpretation of this approximation is that the medium is undergoing deformation but remains stationary.

For Eq.2.16, the same assumptions for material derivative are made, i.e. it is replaced by partial derivative and quadratic term $c(\nabla p)^{2}$ is neglected. 
These assumptions lead to the following equation in place of Eq.2.15 and Eq.2.16:

$\nabla \bullet\left(\frac{k}{\mu} \nabla p\right)=\phi c_{t, I} \frac{\partial p}{\partial t}+\left(c_{b}-c_{s}\right) \frac{d \sigma_{m}}{d t}$

where

$\phi c_{t, I}=\phi c-(1+\phi) c_{s}+c_{b}$

Now, a relationship between $p$ and $\sigma_{m}$ will be established through Poroelastic theory to completely define Eq.2.17.

\subsubsection{Linear Poroelastic Theory: Basic Relations}

Fluid-solid coupling is described by Poroelastic theory that was developed by Biot $^{20-26}$. Some assumptions are made like isothermal conditions and perfectly elastic medium. There are three basic relations that are parallel with the mass balance, Darcy's law and equation of state for modeling of fluid flow. These relations are:

- Stress equilibrium:

$$
\sum_{j=1}^{3} \frac{\partial \sigma_{i j}}{\partial x_{j}}=0
$$

- Strain-displacement relation:

$$
\varepsilon_{i j}=\frac{1}{2}\left(\frac{\partial u_{i}}{\partial x_{j}}+\frac{\partial u_{j}}{\partial x_{i}}\right)
$$

- Strain-stress-pressure:

$$
\varepsilon_{i i}=\frac{1}{E}\left[\sigma_{i i}-v\left(\sigma_{j j}+\sigma_{k k}\right)\right]+\frac{\alpha}{3 K_{b}} p
$$




$$
\varepsilon_{i j}=\frac{\sigma_{i j}}{2 G}
$$

In above equations, $\sigma_{i j}$ is the component of total stress tensor, $\varepsilon_{i j}$ is the component of bulk strain tensor, $u_{i}$ is the component of solid displacement vector $\mathbf{u}\left(u_{x}\right.$, $\left.u_{y}, u_{z}\right), E$ is Young's Modulus, $G$ is shear modulus and $v$ is Poisson's ratio. In Eq.2.21a, $K_{b}$ is the drained jacketed bulk modulus and $\alpha$ is Poroelastic parameter.

Solving Eq.2.21 for stress:

$$
\sigma_{i j}=2 G \varepsilon_{i j}+\lambda \varepsilon_{k k} \delta_{i j}-\alpha p \delta_{i j} .
$$

here $\lambda$ is Lame's constant:

$$
\lambda=\frac{3 v K_{b}}{1+v}=\frac{2 v G}{1-2 v}=K_{b}-\frac{2}{3} G
$$

Adding the three equations 2.21a, 2.21b and 2.22:

$$
e=\frac{\sigma_{m}+\alpha p}{\lambda+(2 / 3) G}=\frac{\sigma_{m}+\alpha p}{K_{b}}
$$

where

$$
\begin{aligned}
& e=\varepsilon_{x x}+\varepsilon_{y y}+\varepsilon_{z z} \ldots \ldots . . \\
& \sigma_{m}=\frac{\sigma_{x x}+\sigma_{y y}+\sigma_{z z}}{3} .
\end{aligned}
$$

here, $e$ is volume strain of solid and $\sigma_{m}$ is the mean total stress. The solid displacement velocity $v_{s}$ and volume strain $e$ are related to solid displacement $u$ by: $v_{s}=\frac{d e}{d t} ;$ $e=\nabla \bullet u$

The divergence of solid velocity is related to the volume strain $e$ by: 
$\nabla \cdot v_{s}=\frac{d e}{d t}=\frac{1}{V_{b}} \frac{d V_{b}}{d t} ; \quad d e=\frac{d V_{b}}{V_{b}}$

\subsubsection{Governing Equation}

The total stresses given by Eq.2.22 should satisfy the equilibrium relation satisfied by Eq.2.19. So substituting Eq.2.22 into Eq.2.19 and applying Eq.2.20, we get:

$\sum_{j=1}^{3} \frac{\partial}{\partial x_{j}}\left\{G\left(\frac{\partial u_{i}}{\partial x_{j}}+\frac{\partial u_{j}}{\partial x_{i}}\right)\right\}=\frac{\partial(\alpha p-\lambda e)}{\partial e}$

These are three equations in $x, y$, and $z$ directions. Simplifying Eq.2.29 for constant elastic coefficients, $G, \lambda$ and $\alpha$ gives $^{19}$ :

$G \nabla^{2} u_{i}+(G+\lambda) \frac{\partial e}{\partial x_{i}}=\alpha \frac{\partial p}{\partial x_{i}}$

Also, eliminating the mean stress $\sigma_{m}$ between Eq.2.24 and Eq.2.17 results in ${ }^{19}$ :

$\nabla \bullet\left(\frac{k}{\mu} \nabla p\right)=\phi c_{t, I I} \frac{\partial p}{\partial t}+\alpha \frac{d e}{d t}$

where

$\phi c_{t, I I}=\phi c_{t, I}-\alpha^{2} c_{b}=\phi c+(\alpha-\phi) c_{s}$

Eq.2.30 and Eq.2.31 are four equations in four unknowns, $p, u_{x}, u_{y}$ and $u_{z}$. This coupled system governs the time history of the deformation and the pressure field.

A more concise representation can be obtained by adding three equations $(\mathrm{x}, \mathrm{y}, \mathrm{z})$ of Eq.2.30:

$G \nabla^{2} u+(G+\lambda) \nabla \nabla \bullet u=\alpha \nabla p$ 
The above highlighted derivations show two major concepts in achieving the coupling between fluid flow and geomechanics. The first concept is interpretations of various rock compressibilities as discussed in Eq.2.13 and Eq.21.4. The second concept is fundamental stress-strain-pressure relations given by Eqs. 2.21, 2.22 and 2.24. Concept of effective stress (introduced by Terzaghi ${ }^{29}$ ) will now be introduced which is implicit in the relations shown by these three equations.

\subsubsection{Effective Stress Concept}

The effective stress is defined as:

$$
\sigma_{i j}^{e}=\sigma_{i j}+\alpha p \delta_{i j}
$$

This effective stress represents the portion of the total stress which is in excess of some fraction of the stress caused by fluid pressure. The above definition of effective stress eliminated the explicit role of fluid pressure in stress-strain relationship. The governing equations of poroelastic theory can be written in terms of effective stress. Eq.2.21 will be written as:

$$
\begin{aligned}
& \varepsilon_{i i}=\frac{\left[\sigma_{i i}^{e}-v\left(\sigma_{j j}^{e}+\sigma_{k k}^{e}\right)\right]}{E} \\
& \varepsilon_{i j}=\frac{\sigma_{i j}^{e}}{2 G} \ldots \ldots \ldots \ldots \ldots \ldots
\end{aligned}
$$

Similarly Eq.2.22 and 2.24 can be written as:

$$
\begin{aligned}
& \sigma_{i i}^{e}=2 G \varepsilon_{i j}+\lambda e \delta_{i j} \\
& e=\frac{\sigma_{m}^{e}}{K_{b}} \ldots \ldots \ldots \ldots . .
\end{aligned}
$$


where $\sigma_{m}^{e}$ is the effective mean normal stress defined as:

$$
\sigma_{m}^{e}=\sigma_{m}+\alpha p=\frac{\left(\sigma_{x x}^{e}+\sigma_{y y}^{e}+\sigma_{z z}^{e}\right)}{3}
$$

The above interpretations show that the elastic behavior and the mechanical properties of a porous fluid bearing rock are assumed to be governed by the effective stresses (single variable) instead of total stress and pore pressure (two variables). $\alpha$ is known as poroelastic constant or Biot's constant which determines the relative contribution of pore pressure on the elastic bulk behavior of a porous medium. The expression for $\alpha$ from bulk volume strain point of view is ${ }^{26,27,30}$ :

$$
\alpha=1-\frac{K_{b}}{K_{s}}=1-\frac{c_{s}}{c_{b}}
$$

The effective stress given by Eq.2.34 is a linear combination of total stress and fluid pressure because in the theory of linear, isotropic poroelasticity, $\alpha$ is considered constant.

\subsection{Nondeformable Porous Media}

The condition for nondeformable porous medium is zero solid velocity i.e. $v_{s}=0$. This means that bulk volume will remain constant and there will no volumetric strain. So following relations will be achieved:

- $\quad d V_{b}=0$ and $d e=0$

- $\frac{d(.)}{d t}=\frac{\partial(.)}{\partial t}$

- $d \sigma_{m}=-\alpha d p$ 
So Eq.2.11 reduces to:

$$
\nabla \bullet\left(\rho \frac{k}{\mu} \nabla p\right)=\phi \rho\left(\frac{1}{\rho} \frac{\partial \rho}{\partial t}+\frac{1}{V_{p}} \frac{\partial V_{p}}{\partial t}\right)
$$

Simplifying the equation further we get:

$$
\nabla \bullet\left(\frac{k}{\mu} \nabla \rho\right)=\phi\left(c+c_{p b}\right) \frac{\partial \rho}{\partial t}
$$

where

$c_{p b}=\frac{1}{V_{p}}\left(\frac{\partial V_{p}}{\partial p}\right)_{V_{b}}=\frac{1}{\phi}\left(\frac{\partial \phi}{\partial p}\right)_{V_{b}}$

Equation 2.41 shows that fluid density satisfies a decoupled diffusion-type equation for a medium of constant bulk volume but with a pressure dependent porosity. The fluid density $\rho$ can be replaced by fluid pressure $p$ if fluid is assumed to be slightly compressible.

\subsection{Type of Coupling}

There are three basic types of algorithms for simulation of geomechanics and fluid flow in reservoirs ${ }^{32}$ depending on the degree of coupling between multiphase flow equations and stress equations. These are full coupling, loose coupling and one-way coupling. 


\subsubsection{Full Coupling}

In a fully coupled simulator, flow variables such as pressure temperature and geomechanical response (displacements) are calculated simultaneously through a system of equations with pressure, temperature and displacements as unknowns. The traditional porous flow equations for a rigid matrix are modified to include terms for mechanical deformation. This type of coupling is sometimes called implicit coupling because the whole system is discretized on one grid domain and solved simultaneously. The derivation of equations governing fluid flow in deformable porous medium in previous section is an example of fully coupled equations. Full coupling is often the preferred method for simulating multiple types of physics simultaneously since it should theoretically produce the most realistic results. Unfortunately, deriving a fully coupled multiphase flow simulator that models nonlinear, inelastic mechanical deformation is extremely difficult. Thus with fully coupled models, one generally simplifies to single phase flow and linear elasticity.

\subsubsection{One-way Coupling/ Explicit Coupling}

Explicit coupling provides the weakest link between reservoir flow and geomechanics deformation. Two separate sets of equations are solved independently over the same total time interval and periodically, output from simulator is passed as input to the geomechanics module in one-way only. This means pore pressure is sent from the flow code to load the mechanics calculation of stresses, strains and displacements; 
however, no information is passed from mechanics to flow. So, change in the porepressure field induces change in stresses and strains but change in the stress and strain field do not affect pore-pressure and rock properties like porosity and permeability. One can often gain valuable information about rock deformation and subsidence from oneway coupling and it is preferable to fluid flow alone.

\subsubsection{Loose Coupling/Iterative Coupling}

This is most common type of coupling which is adopted by many commercial simulators. In loose coupling, reservoir flow variables and geomechanics variables are solved independently and sequentially by a reservoir simulator and a geomechanics module and information is passed at designated time intervals in both directions between the two simulators (fluid flow and geomechanics). Loose coupling has the advantage of being relatively simple to implement like one-way coupling and it captures complex nonlinear physics like fully coupled approach.

ECLIPSE 300® which is being used for geomechanical study for the present work employs loose coupling algorithm. A loose coupling algorithm is described below to highlight the steps and procedure for study of the reservoir geomechanics.

\subsubsection{Loose Coupling Algorithm}

Figure 2 is the flow chart for the loose coupling algorithm ${ }^{32}$. The reservoir simulator and the geomechanical module are coupled by invoking one simulator and then 
the other repeatedly over the total simulation time. Time step for switching from flow to mechanics is decided by the user. The flow simulator runs for the designated time interval $\Delta t_{1}$ where the time $\Delta t_{1}$ will be broken into several time steps. At the end of the time interval $\Delta t_{1}$, the pore pressure is passed to the mechanical code and the mechanics code then runs the simulation for that same (prior) $\Delta t_{1}$ time interval. The geomechanics code can take only one time step for this time interval (or atleast different sub-time steps relative to the flow simulator). Pore pressure input to the geomechanics code, allows calculation of strains and ultimately updates porosity and permeability for the flow simulator's subsequent time steps. Using the updated flow parameters from the mechanics (time step $\Delta t_{1}$ ), fluid flow is simulated for the next time interval $\Delta t_{2}=t_{2}-t_{1}$. The two-way staggered-in-time coupling algorithm proceeds until both simulators reach the final time $t_{\text {end }}$.

Between the flow and geomechanics simulations in the flow chart, there is a column for mapping output and input quantities from one simulator to the other. This column is necessary because the loose coupling algorithm does not require the two simulators to use the same computational grid. One of the advantages of loose coupling is that geomechanics and flow need not have identical spatial grids. The flow simulator should only model the reservoir while geomechanics code may need to extend further in lateral directions than the reservoir and also need to extend up to the earth surface for overburden loading and may cover an area below the reservoir. Here an important thing to note about ECLIPSE $300 \circledR$ is that it uses the same grid for reservoir simulation and geomechanics calculations. So there is no such requirement of mapping out and input quantities from one simulator to the other. 


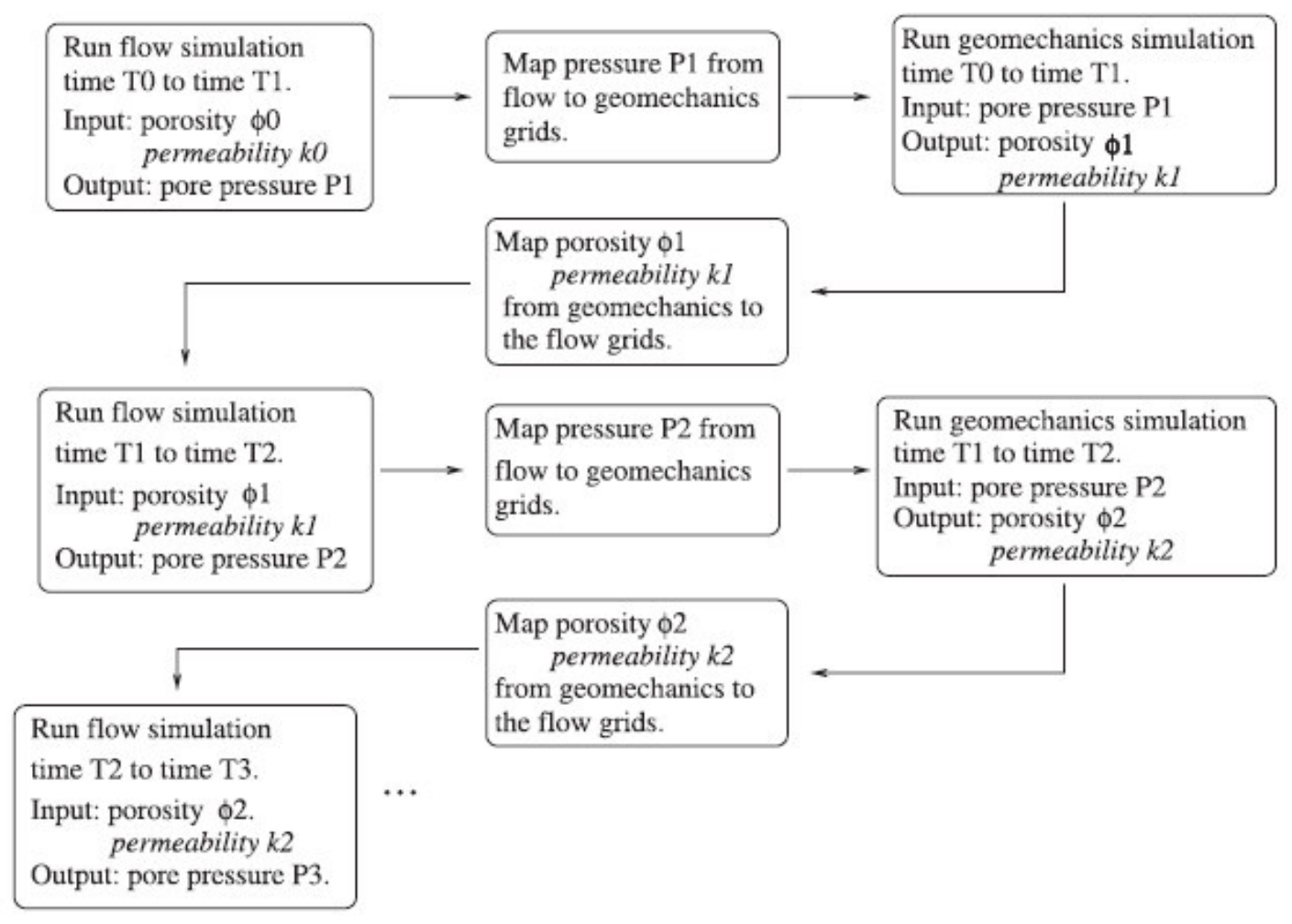

Figure 2 - Flow Chart for Lose Coupling Algorithm ${ }^{32}$

\subsection{Modifications for Simulation}

\subsubsection{Modifications to Fluid Flow: Porosity Updates}

In traditional flow simulators, small changes to porosity $\phi$ is accounted for by following linear expression:

$$
\phi=\phi^{*}\left\lfloor 1+c_{r}\left(P-P^{*}\right)\right\rfloor
$$

In the coupled simulator, Eq.2.43 is not used to determine porosity. Porosity values at the beginning of simulation time step are input to the flow simulator from geomechanics module. The nonlinear system of equations resulting from the finite 
difference discretization of mass conservation equations is solved via Newton's method. This system is written in matrix form, $J x=-R$, with $J$ the Jacobian matrix of partial derivatives with respect to primary variables $x$. and $R$, the residual. The residual for mass balance system for component $\alpha$ is written as:

$$
R_{\alpha}=\phi\left(N_{\alpha}^{n+1}-N_{\alpha}^{n}\right)+d t\left(\nabla \cdot U_{\alpha}^{n+1}-q_{\alpha}^{n+1}\right)
$$

In case of coupled geomechanics and reservoir simulation, porosity updates are introduced at time step $n+l$ through residual modification:

$$
R_{\alpha}=\left(\phi N_{\alpha}\right)^{n+1}-\left(\phi N_{\alpha}\right)^{n}+d t\left(\nabla \cdot U_{\alpha}^{n+1}-q_{\alpha}^{n+1}\right)
$$

So, the porosity values at time step $n+1$ are provided by the geomechanics module and directly incorporated into the Newton system to be solved. In above formulation, mass is conserved and the Newton update accounts for a decrease in pore space by increasing pressures at the next time-step.

\subsubsection{Modifications to Fluid Flow: Permeability Updates}

Dynamic updates of absolute porosity during flow simulation impact large portions of the code. The grid block transmissibilities in the discretized equations and parts of well model must also be continuously updated. Rewriting Eq.2.45 for water phase:

$$
\frac{\left(\phi N_{w}\right)^{n+1}-\left(\phi N_{w}\right)^{n}}{\Delta t^{n+1}}+\nabla \cdot U_{w}^{n+1}=q_{w}^{n+1}
$$

The corresponding nonlinear residual equation in Newton's method is:

$$
R_{w, i j k}^{l}=V_{i j k}\left\{\left(\phi N_{w}\right)_{i j k}^{l}-\left(\phi N_{w}\right)_{i j k}^{l}+\Delta t^{n+1}\left[\nabla \cdot U_{w}-q_{w}\right]_{i j k}^{l}\right\} .
$$


In the above equation, ijk refers to grid cell index, the superscript $l$ refers to the $t^{\text {th }}$ iteration to the superscripted quantity at the time level $n+l$ and $V_{i j k}$ denotes the volume of grid cell $i j k$.

The component mass velocities in the residual equation 2.47 are discretized after including Darcy's law:

$$
\begin{aligned}
\Delta t^{n+1} V_{i j k}\left(\nabla U_{w} .\right)_{i j k}^{l}= & -\lambda_{w, i+1 / 2, j k}^{l}\left[P_{w, i+1, j k}^{l}-P_{w, i j k}^{l}-\rho_{w, i+1 / 2, j k}^{l} g\left(D_{i+1, j k}-D_{i j k}\right)\right] \\
& +\lambda_{w, i-1 / 2, j k}^{l}\left[P_{w, i j k}^{l}-P_{w, i+1, j k}^{l}-\rho_{w, i-1 / 2, j k}^{l} g\left(D_{i j k}-D_{i-1, j k}\right)\right] \\
& + \text { terms for y and zdirections }
\end{aligned}
$$

The transmissibility constants that appear in the upstream weighted mobility computation for $\lambda$ are:

$$
\lambda_{w, i+1 / 2, j k}^{l}=\Delta t^{n+1} 2 \Delta y_{j} \Delta z_{k}\left(\frac{k_{r w}}{B_{w} \mu_{w}}\right)_{i j k}^{l}\left(\frac{\Delta x_{i}}{k_{x x, i j k}}+\frac{\Delta x_{i+1}}{k_{x x, i+1 j k}}\right)^{-1}
$$

where, $k_{x x}, k_{y y}$, and $k_{z z}$, are harmonically averaged values of the absolute permeability tensor between adjacent grid cells and $\Delta x_{i}, \Delta y_{j}$ and $\Delta z_{k}$ are the edge lengths of a rectangular grid cell $i j k$.

The values of transmissibility are generally assumed fixed in uncoupled flow simulators. Here, they must be updated after each geomechanics time-step to account for the permeability changes induced by strain.

\subsubsection{Modifications to Geomechanics: Porosity Updates}

The geomechanics code calculates point-wise changes in pore volume in the reservoir due to hydrocarbon production, pressure decrease and compaction ${ }^{4}$. The 
calculation is achieved through reservoir simulator sending pore pressure field to the geomechanics module where it is used for calculation of total stress:

$$
\sigma_{i j}^{T}=\sigma_{i j}^{e}-p \delta_{i j}
$$

here, $p$ is the pore fluid pressure, $\sigma_{i j}^{e}$ is effective stress and $\sigma_{i j}^{T}$ is total stress. The total stress is used in the determination of the equilibrium state for the reservoir subjected to overburden loads, kinematic boundary conditions and changing pore pressure field. On output, ECLIPSE $300 \circledR$ geomechanics module provides an updated porosity at current time step through default porosity-stress relation which is conservation of rock volume in the grid block over the time or report step:

$V^{n+1}\left(1-\phi^{n+1}\right)=V^{n}\left(1-\phi^{n}\right)$

where $V$ is the bulk volume. This is equivalent to the relation $\delta \phi=-(1-\phi) \delta \varepsilon_{b}$. Here $\delta \varepsilon_{b}$ is change in volumetric strain.

A second porosity-stress relationship model is also available which is based on rock mass conservation:

$$
\delta \phi=-\left(1-\phi-\frac{c_{r}}{c_{b}}\right)\left(\delta \varepsilon_{b}-c_{r} \delta p\right)
$$

The symbol $\delta A$ denotes $A^{n+1}-A^{n}$ where A is any quantity that changes from time step $n$ to time step $n+1$.

\subsubsection{Modifications to Geomechanics: Permeability Updates}

ECLIPSE $300 ®$ modifies permeability as a function of stress ${ }^{4}$. A permeability multiplier as a tabular function of maximum and minimum principal stress is provided as 
input to the coupled simulator. The permeability multipliers after every rock stress updates are calculated to update the grid block permeabilities.

\subsection{Description of Reservoir Simulator}

Flow simulation is carried out for integrated reservoir study in order to predict performance of the oil field and understand reservoir processes such as multiphase fluid flow, rock geomechanics and geochemical response. Brief overview of the simulators used for the study is described below.

\subsubsection{Compositional Flow Simulation}

ECLIPSE $300 ®$ is a compositional simulator with cubic equation of state developed by Schlumberger. ECLIPSE $300 ®$ can be run in fully implicit mode, IMPES and adaptive implicit (AIM) mode. The simulator solves governing equations for multicomponent and multiphase flow in permeable media to compute phase pressures and fluxes that form the basis for tracing streamline trajectories. ECLIPSE 300 incorporates 4 different equations. When an equation of state is selected, it is used to obtain Z-factors and phase fugacities to define inter-phase equilibrium and fluid densities. 


\subsubsection{Flash Calculation}

The ECLIPSE 300 iteration process to converge a solution consists of linear, nonlinear and flash iteration. The final solution at the end of a time-step for each grid block consists of a pressure; water, oil and gas saturations; liquid and vapor molar fractions; and mole factions of each component in the liquid and vapor phase.

\subsubsection{Flux Calculation}

Streamline tracing is done based on the total velocity and phase velocities across the grid cells but ECLIPSE $300 ®$ does not report flux values for the three phases i.e. oil, gas and water. ECLIPSE $300 ®$ calculates flow rate of a component $\mathrm{c}$ embedded in a phase $p(\mathrm{p}=\mathrm{o}, \mathrm{w}, \mathrm{g})$ into cell $i$ from a neighboring cell $n$ by following relation ${ }^{4}$ :

$$
F_{p n i}^{c}=T_{n i} M_{p}^{c} d P_{p n i}
$$

$M_{p}^{c}$ is generalized mobility of component $c$ in phase $p$ and is given by:

$$
M_{p}^{c}=x_{p}^{c} k_{r p}\left(S_{p}\right) \frac{b_{p}^{m}}{\mu_{p}}
$$

here, $x_{p}^{c}$ is mole fraction of component $c$ in phase $p$

and $d P_{p n i}$ is the potential difference of phase $p$ between cells $n$ and $i$ given by:

$$
d P_{p n i}=P_{n}-P_{i}+P_{c p n}-P_{c p i}-\rho_{p n i} g\left(D_{n}-D_{i}\right)
$$

The net flow rate of component $c$ from cell $i$ into neighboring cells is obtained by summing over all phases over the neighboring cells: 


$$
F_{i}^{c}=\sum_{n} F_{p n i}^{c}
$$

Steps to calculate phase fluxes and total flux ${ }^{2}$ :

- Fractional flow of a component $c$ in a particular phase $p$ is calculated by taking ratio of mobility of the component in that phase divided by sum of the mobility of a component in all phases

$$
f_{p}^{c}=\frac{x_{p}^{c} k_{r p}\left(S_{p}\right) \frac{b_{p}^{m}}{\mu_{p}}}{x_{o}^{c} k_{r o}\left(S_{o}\right) \frac{b_{o}^{m}}{\mu_{o}}+x_{g}^{c} k_{r g}\left(S_{g}\right) \frac{b_{g}^{m}}{\mu_{g}}+x_{w}^{c} k_{r w}\left(S_{w}\right) \frac{b_{w}^{m}}{\mu_{w}}}
$$

- Flow rate of the component $c$ embedded in phase $p$ is computed by multiplying fractional flow of the component found in previous step with net flow rate of component $c$ reported by ECLIPSE 300 in the Restart files.

$$
F_{p n i}^{c}=f_{p}^{c} F_{i}^{c}
$$

- Phase flux is calculated by summing flow rate over all the components existing in the phase.

$$
F_{p}=\sum_{c=1}^{c=n} F_{p n i}^{c}
$$

- Total flux is then obtained by adding all phase fluxes.

\subsubsection{Partly Coupled Geomechanical Simulation}

The Geomechanics option is included in ECLIPSE 300. This option provides improved ability to simulate compaction processes that are not available with the 
standard rock compaction option. Coupling of geomechanical rock stress calculation with the fluid flow has several benefits like ${ }^{4}$ :

1. Improved ability to predict subsidence and compaction in a reservoir.

2. Improved modeling of fluid flow through stress dependent permeability and Biot's constant.

3. Flexibility:

- Stress, traction and displacement boundary conditions can be set on external as well as internal surfaces.

- The grid for the stress calculation is the same grid that is used when calculating fluid flow.

- Rock mechanical properties are input on a grid by grid basis.

A finite element method is used to calculate the stress.

\subsection{2 .1 Elastic Stress Equations}

Steady state rock momentum balance equations ${ }^{14,15}$ in the $\mathrm{x}, \mathrm{y}$ and $\mathrm{z}$ directions used in coupled geomechanical simulator by ECLIPSE $300 ®$ can be written as:

$$
\begin{aligned}
& \frac{\partial \sigma_{x}}{\partial x}+\frac{\partial \tau_{y x}}{\partial y}+\frac{\partial \tau_{z x}}{\partial z}=0 \\
& \frac{\partial \sigma_{y}}{\partial y}+\frac{\partial \tau_{x y}}{\partial x}+\frac{\partial \tau_{z y}}{\partial z}=0 \\
& \frac{\partial \sigma_{z}}{\partial z}+\frac{\partial \tau_{x z}}{\partial x}+\frac{\partial \tau_{y z}}{\partial y}+r g=0
\end{aligned}
$$


here, $r$ is the rock density or a combination of rock and reservoir density and $g$ is gravitational constant.

The elastic normal stresses $\sigma$ and shear stresses $\tau$ can be expressed $\mathrm{n}$ terms of strains, $\mathcal{E}$ and $\gamma$, as:

$$
\begin{aligned}
& \sigma_{x}=2 G \varepsilon_{x}+\lambda\left(\varepsilon_{x}+\varepsilon_{y}+\varepsilon_{z}\right) \delta_{i j}-\alpha P-(2 G+3 \lambda) \alpha_{T}\left(T-T_{r}\right) \\
& \sigma_{y}=2 G \varepsilon_{y}+\lambda\left(\varepsilon_{x}+\varepsilon_{y}+\varepsilon_{z}\right) \delta_{i j}-\alpha P-(2 G+3 \lambda) \alpha_{T}\left(T-T_{r}\right) \\
& \sigma_{z}=2 G \varepsilon_{z}+\lambda\left(\varepsilon_{x}+\varepsilon_{y}+\varepsilon_{z}\right) \delta_{i j}-\alpha P-(2 G+3 \lambda) \alpha_{T}\left(T-T_{r}\right) \\
& \tau_{x y}=G \gamma_{x y} \\
& \tau_{y z}=G \gamma_{y z} \\
& \tau_{y z}=G \gamma_{y z}
\end{aligned}
$$

Constants $G$ (modulus of shear) and $\lambda$ are Lame's constant. They are functions of Young's Modulus $E$ and Poisson's ratio $v$.

Strains $\varepsilon_{x, y, z}$ are defined in terms of displacements in the $\mathrm{x}, \mathrm{y}, \mathrm{z}$ directions, namely $u, v$ and $w$ :

$$
\begin{aligned}
& \varepsilon_{x}=\frac{\partial u}{\partial x} \\
& \varepsilon_{x}=\frac{\partial v}{\partial y} \\
& \varepsilon_{z}=\frac{\partial w}{\partial x} \\
& \gamma_{x y}=\frac{\partial u}{\partial y}+\frac{\partial v}{\partial x} \\
& \gamma_{y z}=\frac{\partial v}{\partial z}+\frac{\partial w}{\partial y}
\end{aligned}
$$


$\gamma_{z x}=\frac{\partial w}{\partial x}+\frac{\partial u}{\partial z}$

\subsubsection{Variables and Solution of the Equations}

Variables used in fluid flow simulator are moles of all fluid components per unit pore volume, pressure of one of the fluid phases. Variables used in the stress calculation are rock displacements in the $\mathrm{x}, \mathrm{y}, \mathrm{z}$ directions and porosity. The linear solver used to solve the system of stress equations is an algebraic multi-grid solver. 


\section{CHAPTER III}

\section{STREAMLINE TRACING}

Streamlines are defined as instantaneous lines that are tangential to a velocity field ${ }^{1}$. Streamlines define spatial discretization of the flow field and such discretization places a higher resolution in regions of faster flow. The most important feature of streamlines is time of flight which is defined as the travel time of a neutral tracer particle along a streamline. Streamline tracing starts with first solving for pressure field using finite-difference simulator (ECLIPSE 300) and calculation of Darcy velocity at each of the grid faces. This velocity is used to find time of flight for a particle for traveling from entry face to exit face. Methodology has been described below to find time of flight in grid cells.

\subsection{Pollock's Tracing Algorithm for Rectangular Cells}

To compute time of flight, essentially all streamline codes follow a construction due to Pollock ${ }^{17}$, in which transit time from an initial point in space is built up, one cell at a time. Streamline and time of flight calculation in rectangular cells is briefly discussed below $^{1}$. The discussion will be followed by time of flight calculation for corner point grid geometry.

Steps:

- First, pressure distribution in the reservoir is obtained through finite-difference solution of pressure equations. 
- Once pressure distribution is obtained, volumetric fluxes are calculated using Darcy's law.

Consider a grid block in the finite-difference solution of the pressure equation.

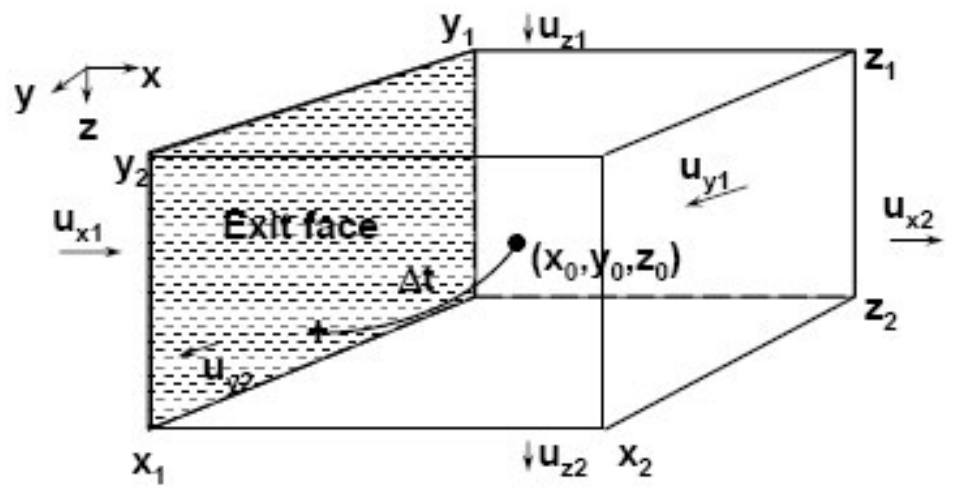

Figure 3 - Grid Block Time of Flight Calculation ${ }^{16}$

The numerical solution gives pressures at the block center and the fluid velocities (fluxes) at the block faces. The fluid velocities of each phase at block face are added to obtain total fluid velocity and this total velocity is used for tracing streamline. Pollock's tracing algorithm assumes that each component of velocity varies linearly between the values on the appropriate pair of cell faces. This leads to following cell velocity models:

$$
\begin{aligned}
& u_{x}=u_{x 1}+c_{x}\left(x-x_{1}\right) \\
& u_{y}=u_{y 1}+c_{y}\left(y-y_{1}\right) \\
& u_{z}=u_{z 1}+c_{z}\left(z-z_{1}\right)
\end{aligned}
$$


The coefficients depend on difference of Darcy velocities on grid block faces:

$$
\begin{gathered}
c_{x}=\frac{\left(u_{x 2}-u_{x 1}\right)}{\Delta x} \\
c_{y}=\frac{\left(u_{y 2}-u_{y 1}\right)}{\Delta y} . \\
c_{z}=\frac{\left(u_{z 2}-u_{z 1}\right)}{\Delta z}
\end{gathered}
$$

where, $u_{x}, u_{y}$ and $u_{z}$ are x-velocity, y-velocity and z-velocity and $\Delta x, \Delta y$ and $\Delta z$ are dimensions of the cells in respective coordinate directions. The streamline trajectory and time of flight within gridblock are computed by direct integration of the cell velocities:

$$
\frac{d \tau}{\phi}=\frac{d x}{u_{x}}+\frac{d y}{u_{y}}+\frac{d z}{u_{z}}
$$

A particle starting at an arbitrary location $\left(x_{0}, y_{0}, z_{0}\right)$ within a gridblock can exit cell through any of the six faces. For linear velocities of Eq.3.1, differential equations in Eq.3.3 can be integrated to obtain time of flight of six faces:

$$
\begin{gathered}
\frac{\Delta \tau_{x i}}{\phi}=\int_{x_{0}}^{x_{1}} \frac{d x}{u_{x 0}+c_{x}\left(x-x_{0}\right)}=\frac{1}{c_{x}} \ln \left(\frac{u_{x i}}{u_{x o}}\right) \\
\frac{\Delta \tau_{y i}}{\phi}=\int_{y_{0}}^{y_{1}} \frac{d y}{u_{y 0}+c_{y}\left(y-y_{0}\right)}=\frac{1}{c_{y}} \ln \left(\frac{u_{y i}}{u_{y o}}\right) . \\
\frac{\Delta \tau_{z i}}{\phi}=\int_{z_{0}}^{z_{1}} \frac{d z}{u_{z 0}+c_{z}\left(z-z_{0}\right)}=\frac{1}{c_{z}} \ln \left(\frac{u_{z i}}{u_{z o}}\right)
\end{gathered}
$$


Pollock's algorithm specifies the correct exit face as the one requiring minimum positive transit time. Thus, the actual cell time of flight for the particle will be given by the minimum over allowable edges.

$\Delta \tau=$ Min Positive $\left(\Delta \tau_{x 1}, \Delta \tau_{x 2}, \Delta \tau_{y 1}, \Delta \tau_{y 2}, \Delta \tau_{z 1}, \Delta \tau_{z 2}\right)$

Knowing the time of flight, its exit coordinates can now be obtained by rearranging Eq.3.4:

$$
\begin{aligned}
& x=x_{0}+u_{x 0}\left(\frac{e^{c_{x} \Delta z / /}-1}{c_{x}}\right) \\
& y=y_{0}+u_{y 0}\left(\frac{e^{c_{y} \Delta z / \phi}-1}{c_{y}}\right) \\
& z=z_{0}+u_{z 0}\left(\frac{e^{c_{z} \Delta z / \phi}-1}{c_{z}}\right)
\end{aligned}
$$

This tracing algorithm is applied to every gridblock where flow of fluid takes place to obtain streamline and time of flight.

\subsection{Extension of Pollock's Algorithm to Corner Point Grid}

Conventional reservoir simulators utilize a complex type of cell geometries than simple rectangles and the velocity fields they model are often more complex than linear. Corner point geometries provide perfect way of describing reservoir geometry as they do not have uniform thickness and cross-sectional area and they are specified by eight corners in $3 \mathrm{D}$. 


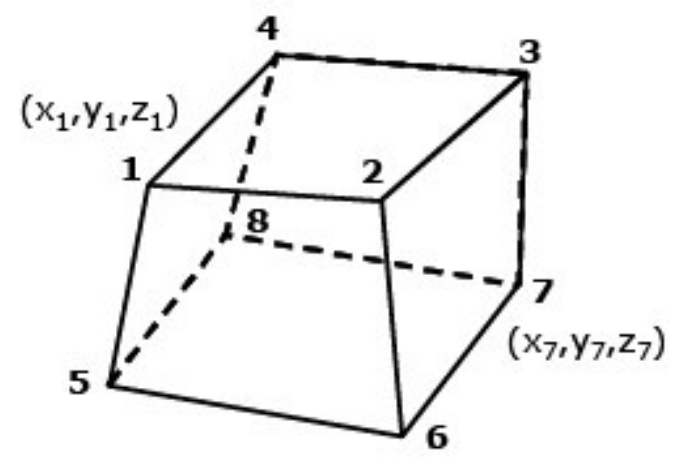

\section{Figure 4 - Corner Point Cell Described by Eight Corners ${ }^{16}$}

Tracing streamlines in such cell geometry becomes challenging task. To deal with this, description of construction due to Cordes and Kinzelback (CK) (1990) in which the corner-point cell is transformed back to a unit cube is given ${ }^{16}$.

Pollock's equation is rewritten in dimensionless variables by using fractional distances in the $x, y$ and $z$ directions.

$$
\alpha=\frac{x}{D X}, \beta=\frac{y}{D Y}, \gamma=\frac{z}{D Z}
$$

Directional Darcy velocities are converted into volumetric fluxes using the crosssectional areas:

$$
Q_{x}=u_{x} . D Y . D Z, Q_{y}=u_{y} . D X . D Z, Q_{z}=u_{z} . D X . D Y
$$

The three velocity equations can be written as:

$$
\begin{aligned}
& \phi . D X . \frac{d \alpha}{d \tau}=\frac{Q_{x}(\alpha)}{D Y . D Z} \\
& \phi . D Y . \frac{d \beta}{d \tau}=\frac{Q_{y}(\beta)}{D X . D Z}
\end{aligned}
$$


$\phi . D Z \cdot \frac{d \gamma}{d \tau}=\frac{Q_{z}(\gamma)}{D X . D Y}$

Combining these relationships,

$\frac{d \tau}{\phi . D X . D Y . D Z}=\frac{d \alpha}{Q_{x}(\alpha)}=\frac{d \beta}{Q_{y}(\beta)}=\frac{d \gamma}{Q_{z}(\gamma)}$.

The above equation is identical to Eq.3.3

A corner point cell is defined as a tri-linear mapping from the unit cube into physical space. Each point in the physical space is considered as the back-transform of a point in unit space by conserving its barycentric coordinate.

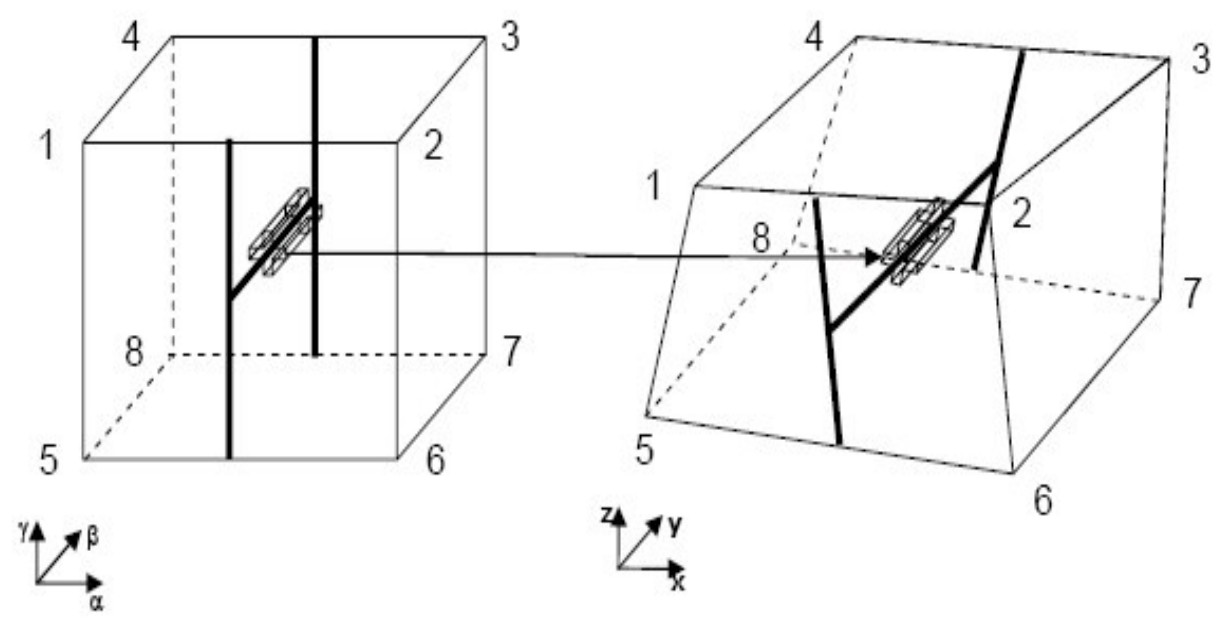

Figure 5 - Isoparametric Transformation ${ }^{16}$

The Jacobian may be used to determine the cell volume, since it is the ratio of physical volume to unit volume.

$$
J(\alpha, \beta, \gamma)=\left\|\frac{\partial(x, y, z)}{\partial(\alpha, \beta, \gamma)}\right\|
$$




$$
\text { Volume }=\int_{(0,0,0)}^{(1,1,1)} J(\alpha, \beta, \gamma) d \alpha . d \beta \cdot d \gamma
$$

To determine the time of flight a velocity model is required within the cell. For a corner point cell in three-dimension, the following velocity model is imposed:

$$
\begin{aligned}
& \phi . \cdot \frac{d \alpha}{d \tau}=\frac{Q_{x}(\alpha)}{J(\alpha, \beta, \gamma)} \\
& \phi . \cdot \frac{d \beta}{d \tau}=\frac{Q_{y}(\beta)}{J(\alpha, \beta, \gamma)} . \\
& \phi . \cdot \frac{d \gamma}{d \tau}=\frac{Q_{z}(\gamma)}{J(\alpha, \beta, \gamma)}
\end{aligned}
$$

In these set of equations each volumetric flux is linearly interpolated in the appropriate direction, similar to Pollock's velocity interpolation,

$$
Q_{j}\left(\alpha_{j}\right)=A_{j}+C_{j} \cdot \alpha_{j,} \quad j=1,2,3 .
$$

In the form of Eq.3.13, the $(\alpha, \beta, \gamma)$ trajectories are much more difficult to integrate than for rectangular cells, as all three parameters are coupled through the Jacobian. The CK solution to Eq.3.13 selects one of the three integrals and then substitute for $\beta(\alpha)$ and $\gamma(\alpha)$. A simpler development is possible with the introduction of a parameter $T$ that increases along a trajectory, and acts as a time-like variable,

$$
d T=\frac{1}{\phi} \frac{d \tau}{J(\alpha, \beta, \gamma)}=\frac{d \alpha}{Q_{1}(\alpha)}=\frac{d \beta}{Q_{2}(\beta)}=\frac{d \gamma}{Q_{3}(\gamma)}
$$




\subsubsection{Pseudo Time of Flight}

Tracing methodology is explained by considering a unit cube as shown

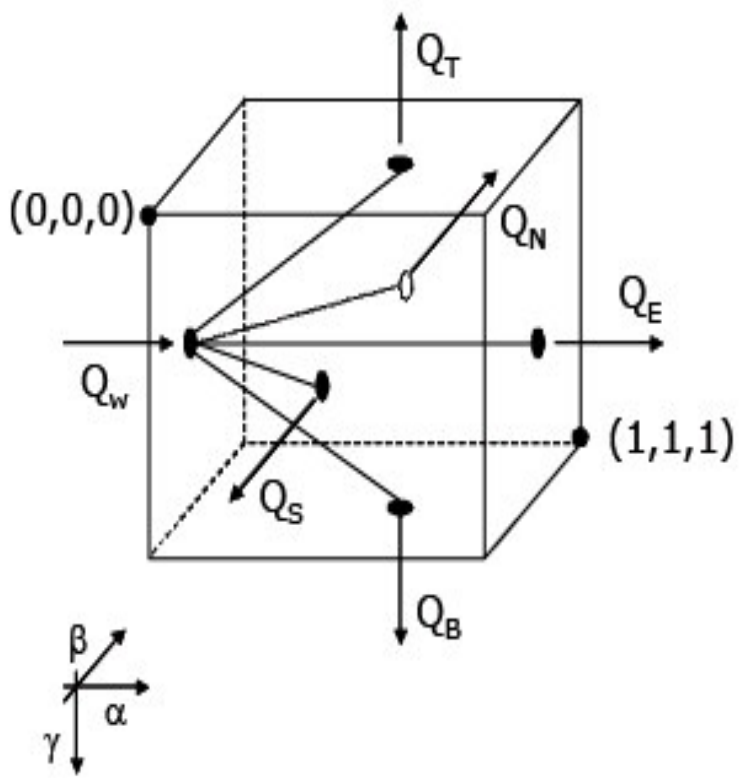

Figure 6 - Schematic Showing the Pseudo Time of Flight in a Unit Cube ${ }^{16}$

- Equation 3.15 is used to determine the time that a particle will require to reach each face.

For x-direction,

$$
\int_{0}^{T_{E}} d T=\int_{\alpha_{0}}^{\alpha} \frac{d \alpha}{Q_{1}(\alpha)}
$$

- The volumetric flux is replaced by its linear interpolate in the x-direction by using Eq.3.14,

$T_{E}=\int_{\alpha_{0}}^{\alpha} \frac{d \alpha}{A_{1}+C_{1} \alpha}=\frac{1}{c_{1}} \ln \left[\frac{a_{1}+\alpha \cdot c_{1}}{a_{1}+\alpha_{0} \cdot c_{1}}\right]$ 
- Similarly time for the particle to reach other faces is calculated and the time required for the particle to reach a boundary face will be the minimum non-negative pseudo time of flight denoted by $T$.

- Once the pseudo time of flight $T$ is known, the exit coordinate of the particle is calculated using Eq.3.15,

$$
\begin{aligned}
& \alpha_{e}=\alpha_{0}+\left(a_{1}+\alpha_{0} \cdot c_{1}\right)\left(\frac{e^{c_{1} T}-1}{c_{1}}\right) \\
& \beta_{e}=\beta_{0}+\left(a_{2}+\beta_{0} \cdot c_{2}\right)\left(\frac{e^{c_{2} T}-1}{c_{2}}\right) \\
& \gamma_{e}=\gamma_{0}+\left(a_{3}+\gamma_{0} \cdot c_{3}\right)\left(\frac{e^{c_{3} T}-1}{c_{3}}\right)
\end{aligned}
$$

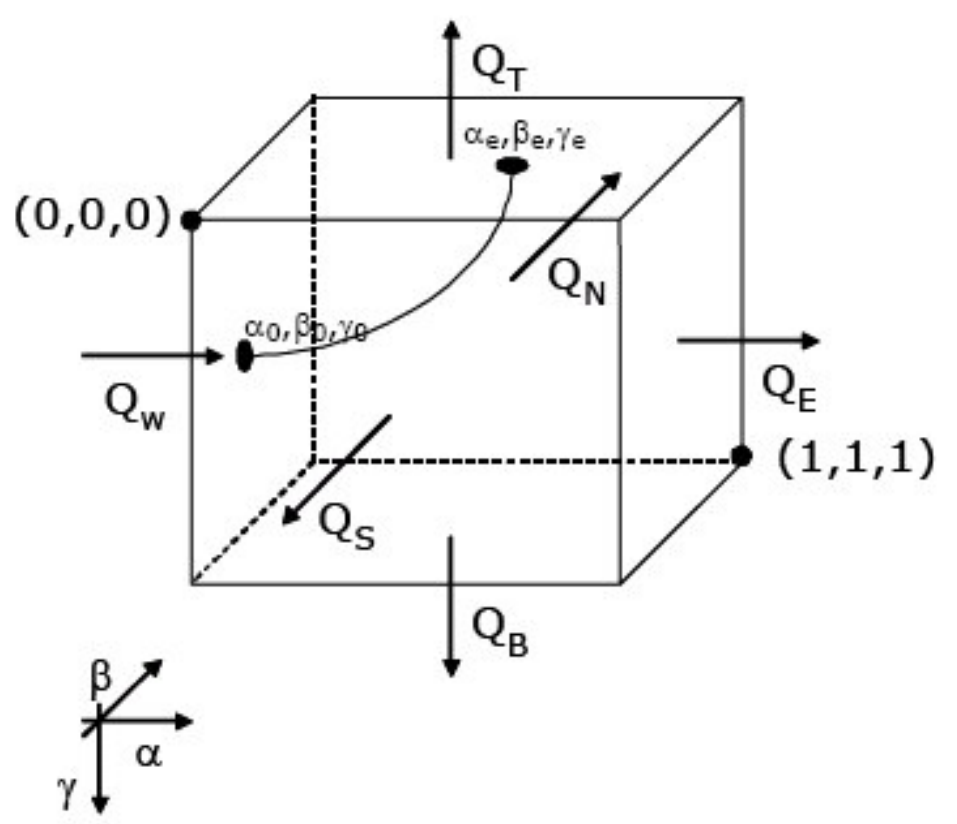

Figure 7 - Exit Coordinates Computation in a Unit Cube ${ }^{16}$ 
- Isoparametric transformation is used to transform this pseudo time of flight to real space. A unit cube is transformed to a CPG grid cell by an isoparametric set of equations

$$
\begin{aligned}
& x=p_{1, x} \alpha+p_{2, x} \beta+p_{3, x} \gamma+p_{4, x} \alpha \beta+p_{5, x} \beta \gamma+p_{6, x} \alpha \gamma+p_{7, x} \alpha \beta \gamma+p_{8, x} \\
& y=p_{1, y} \alpha+p_{2, y} \beta+p_{3, y} \gamma+p_{4, y} \alpha \beta+p_{5, y} \beta \gamma+p_{6, y} \alpha \gamma+p_{7, y} \alpha \beta \gamma+p_{8, y} \\
& z=p_{1, z} \alpha+p_{2, z} \beta+p_{3, z} \gamma+p_{4, z} \alpha \beta+p_{5, z} \beta \gamma+p_{6, z} \alpha \gamma+p_{7, z} \alpha \beta \gamma+p_{8, z}
\end{aligned}
$$

where,

$$
\begin{array}{lrl}
p_{1, x}=x_{2}-x_{1} & p_{2, x}=x_{4}-x_{1} & p_{3, x}=x_{5}-x_{1} \\
p_{4, x}=x_{1}+x_{3}-x_{2}-x_{4} & p_{5, x}=x_{1}+x_{8}-x_{4}-x_{5} & p_{6, x}=x_{1}+x_{6}-x_{2}-x_{5} \\
p_{7, x}=x_{2}+x_{4}+x_{5}+x_{7}-x_{1}-x_{3}-x_{6}+x_{8} \quad p_{8, x} \approx x_{1} \ldots \ldots \ldots \ldots \ldots \ldots \ldots \ldots \ldots \ldots \ldots \ldots \ldots
\end{array}
$$

Similarly, coefficients in terms of $y$ are $p_{1, y}, p_{2, y}, \ldots p_{8, y}$ and in terms of $z$ are $p_{1 z}$, $p_{2, z} \ldots p_{8, z}$

- The time of flight in real space is obtained by carrying out the integral:

$$
\tau=\phi \int_{0}^{T} J(\alpha(T), \beta(T), \gamma(T)) d T
$$

The Jacobian of transformation is given by:

$$
J(\alpha, \beta, \gamma)=\left|\begin{array}{lll}
\frac{\partial x}{\partial \alpha} & \frac{\partial x}{\partial \beta} & \frac{\partial x}{\partial \gamma} \\
\frac{\partial y}{\partial \alpha} & \frac{\partial y}{\partial \beta} & \frac{\partial y}{\partial \gamma} \\
\frac{\partial z}{\partial \alpha} & \frac{\partial z}{\partial \beta} & \frac{\partial z}{\partial \gamma}
\end{array}\right|
$$

The partial derivatives are obtained by differentiating Eq.3.19. 
In the above integral, the Jacobian is a polynomial in $\alpha, \beta$ and $\gamma$ and they in turn are all known functions of the pseudo time of flight. The resulting integrand is a sum of exponentials and constants which can be integrated analytically. 


\section{CHAPTER IV}

\section{APPLICATION AND RESULTS}

\subsection{Synthetic Case Study}

The visualization and comparison of flow patterns for geomechanical simulation and conventional simulation is done for a synthetic case which consists of 4 producers and 1 injection well in a 5 spot pattern. The case consists of compositional fluid with 5 components. In addition to reservoir section, the grid model consists of overburden, underburden, and sideburden to represent surrounding containment for the reservoir. The grid is populated with constant rock parameters like rock density, Young's modulus, Poisson's ratio and porosity. A heterogeneous permeability distribution is used to populate the grid. The model consists of 50 cells in $\mathrm{x}$-direction, 50 cells in y-direction and 20 cells in z-direction. Out of 20 layers, reservoir section comprises of 5 layers.
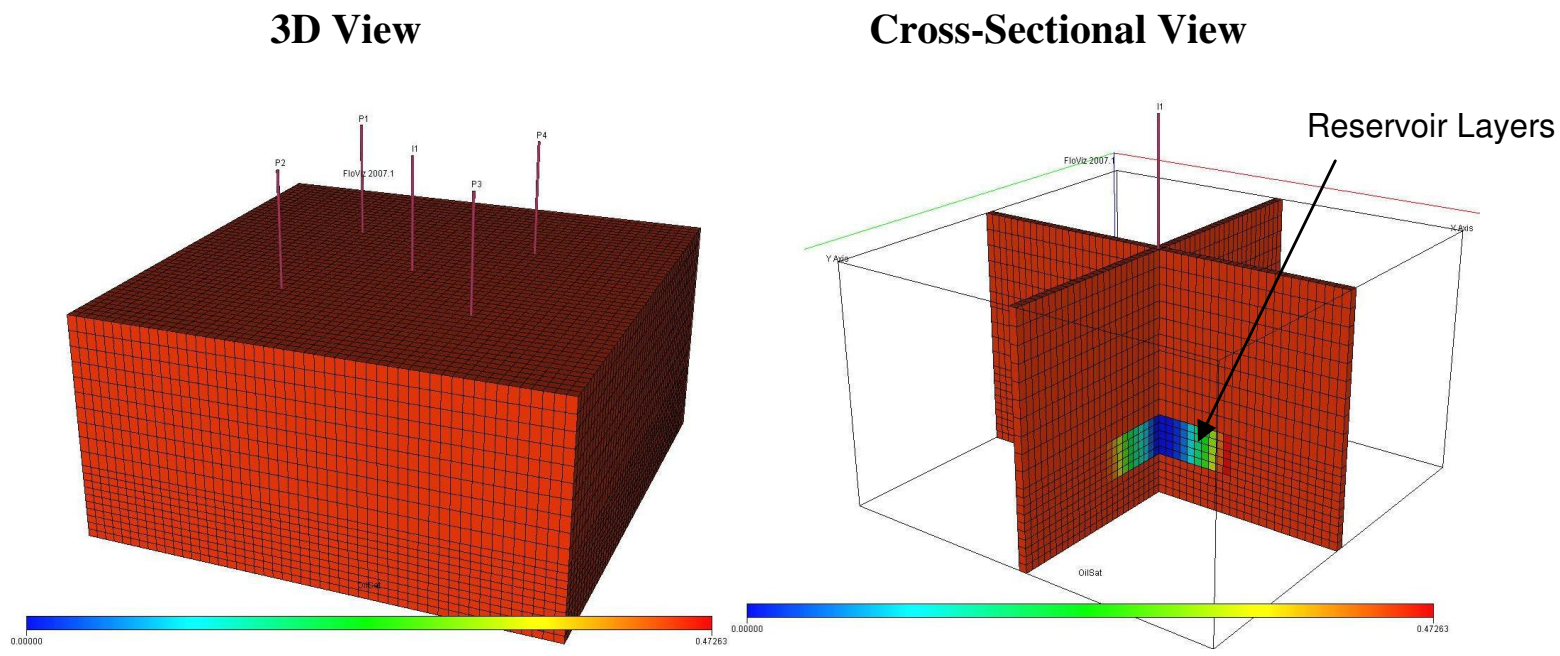

Figure 8 - Grid View 


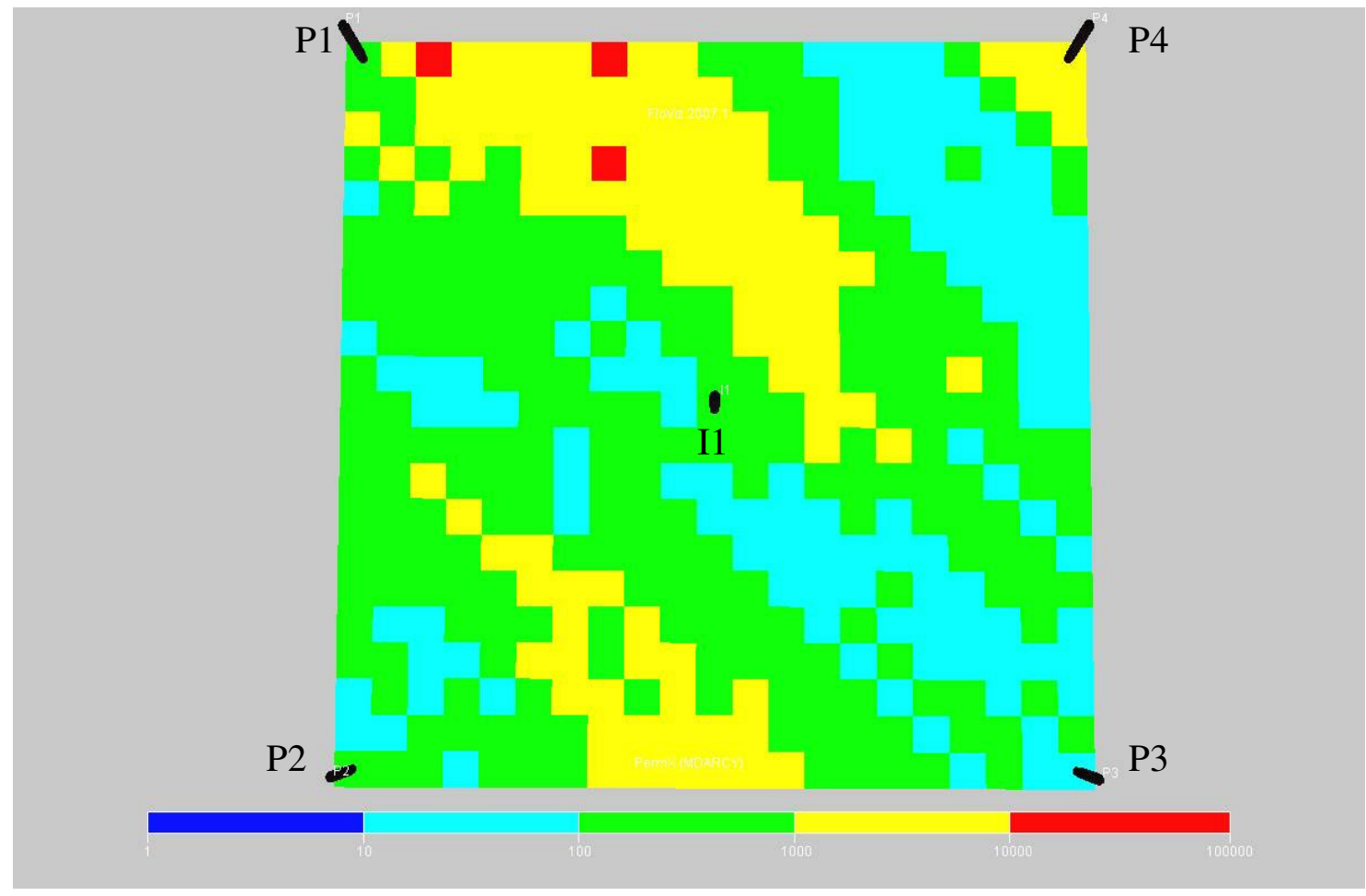

\section{Figure 9 - Top View of Permeability Distribution for the First Reservoir Layer}

The picture above shows the permeability distribution for the first reservoir layer. The distribution shows high permeability trend in the north-west direction. Only the reservoir section has permeability as fluid flow will be taking place inside reservoir and the rest of the model has zero permeability.

The values of various rock mechanical parameters required for geomechanics calculation and other rock parameters have been provided in the Table 1. The rock mechanical parameters values remain same for simulations of the synthetic case and the SPE $9^{\text {th }}$ comparative study. Table 2 shows the input values of the permeability multiplier as a tabular function of maximum and minimum principal stress. The table remains same for simulations of both the case studies. 
Table 1: Input Parameter Values

\begin{tabular}{|l|c|}
\hline \multicolumn{1}{|c|}{ Parameters } & Value \\
\hline Porosity, $\phi$ & $22.5 \%$ \\
\hline Young's Modulus, E, psi & 10000 \\
\hline Poisson's ratio, $\nu$ & 0.43 \\
\hline Rock density, $\rho_{s}$ Ib/cuft & 250 \\
\hline Biot's Constant, $\alpha$ & 1 \\
\hline Lame's constant, $\lambda$ & Default \\
\hline Shear Modulus, $G$, psi & Default \\
\hline Rock Compressibility, $c_{r}, 1 / \mathrm{psi}$ & $4.00 \mathrm{E}-06$ \\
\hline Reference Pressure, $P^{\circ}, \mathrm{psi}$ & 3000 \\
\hline
\end{tabular}

Table 2: Principal Stress vs Permeability Multiplier

\begin{tabular}{|c|c|c|c|c|c|}
\hline \multirow{2}{*}{$\begin{array}{c}\text { Minimum Principal } \\
\text { Stress }\end{array}$} & \multicolumn{5}{|c|}{ Maximum Principal Stress } \\
\cline { 2 - 6 } & -1000 & -750 & -500 & -250 & 100 \\
\hline-1000 & 0.8 & 0.82 & 0.84 & 0.88 & 0.9 \\
\hline-600 & 0.9 & 0.92 & 0.94 & 0.96 & 0.99 \\
\hline-350 & 0.97 & 0.99 & 1.01 & 1.1 & 1.2 \\
\hline-100 & 1.22 & 1.28 & 1.32 & 1.34 & 1.35 \\
\hline 100 & 1.3 & 1.36 & 1.4 & 1.45 & 1.5 \\
\hline
\end{tabular}




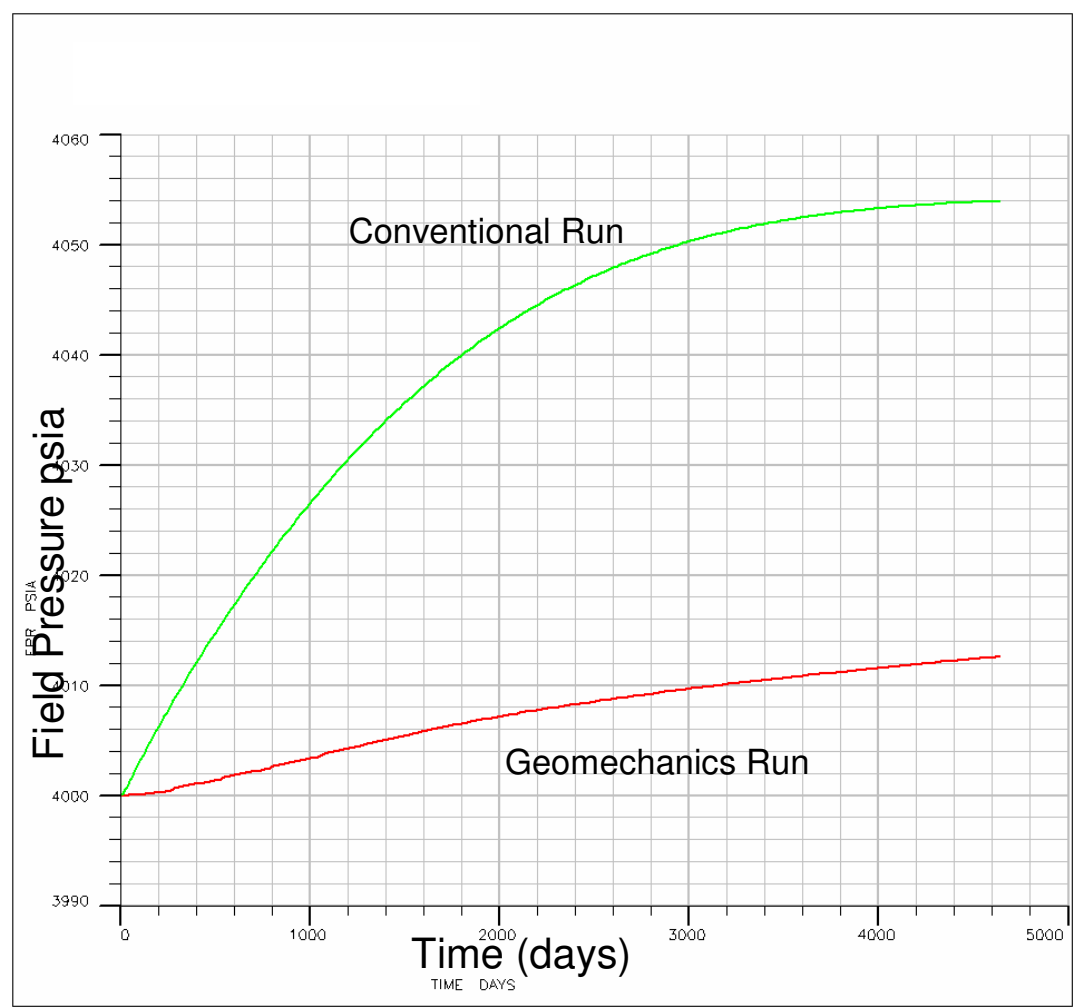

\section{Figure 10 - Field Pressure for Runs With and Without Geomechanics Option}

The synthetic case explained above is specifically a water injection case. Water injection is done in the field for pressure maintenance and the pressure profile for conventional run represents increasing field pressure as a result. There is no noticeable pore volume change for the conventional run but the simulation run with geomechanics option will experience an increase in its pore volume. This pore volume increase offsets the increase in pressure due to water injection causing pressure rise in the reservoir to be less compared to conventional simulation. 


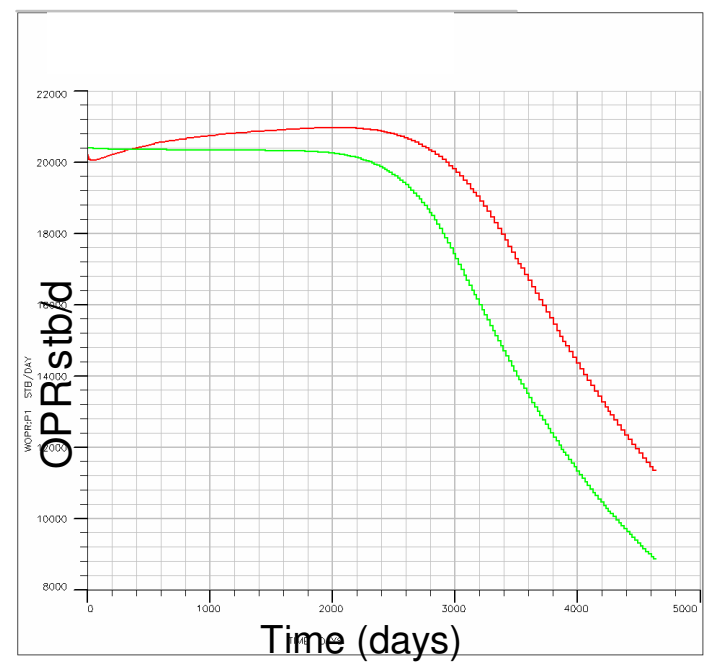

P1 Well

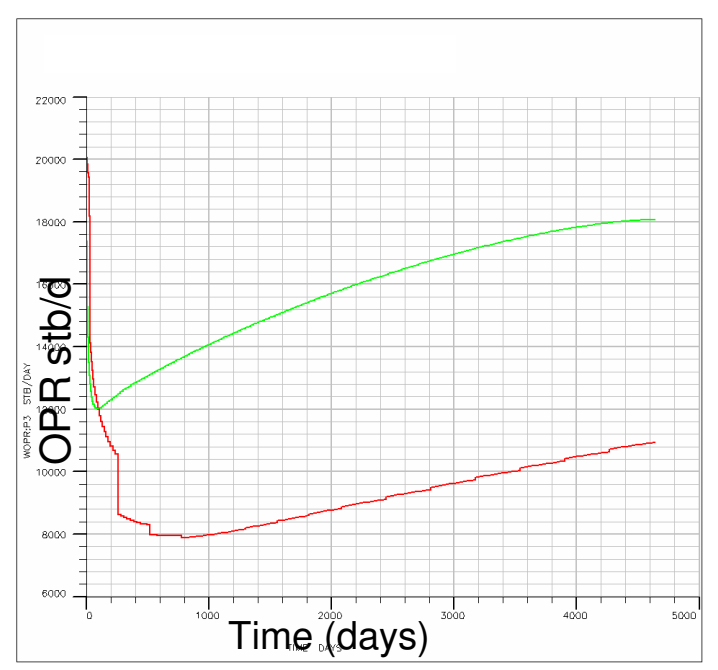

P3 Well

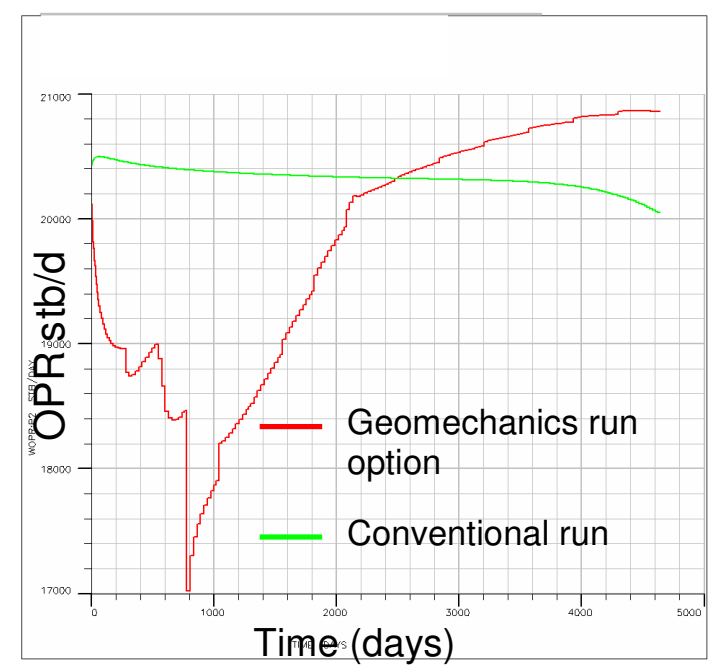

P2 Well

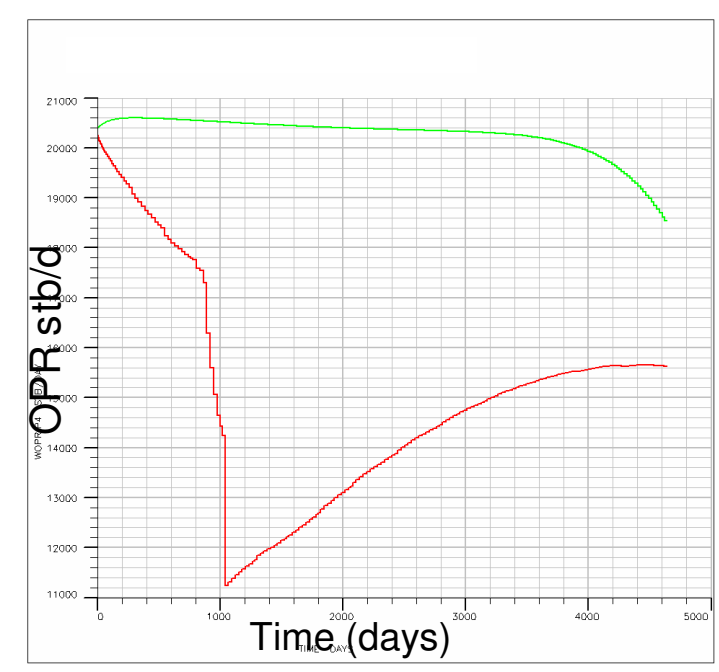

P4 Well

\section{Figure 11 - Plots Showing Oil Production Rate for All the Producers}

Based on Figure 11, it can be inferred that Geomechanics is playing an important role in influencing well flow rates. For all the producers, initial oil flow rates are higher for conventional run (green line) compared to oil flow rates for geomechanics run. This difference is due to rapid pressure change in the conventional simulation which manifests itself as higher flow potential for phases present, leading to higher flow rates. In the run 
with geomechanics option, changes in the pore volume causes pressure change in the reservoir to be slow which leads to low flow potential and subsequently low flow rates. Streamline time of flight figures will be highlighted later to further explain trends seen in the plots for all the producers.

\section{$1^{\text {st }}$ Report-Step}

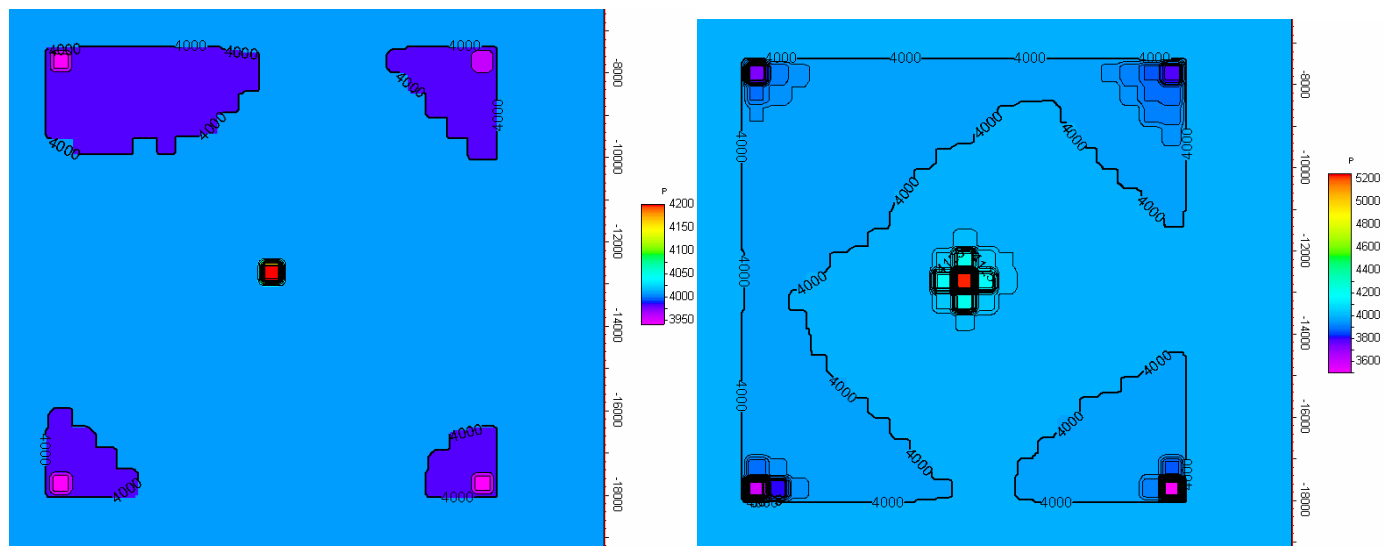

Figure 12 - Pressure Contour

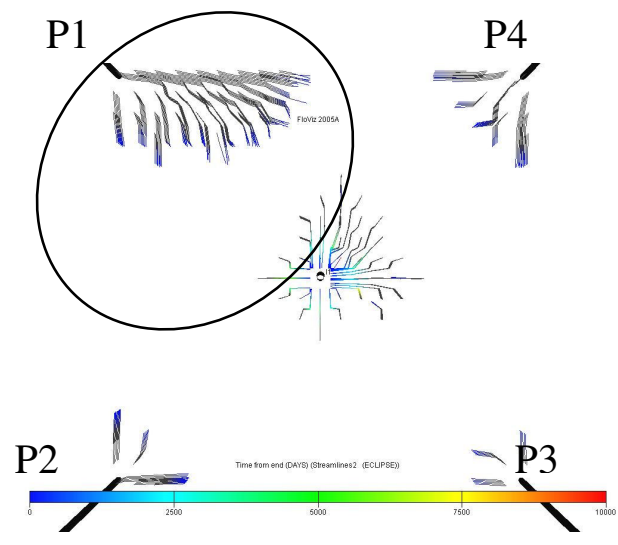

Geomechanics Run

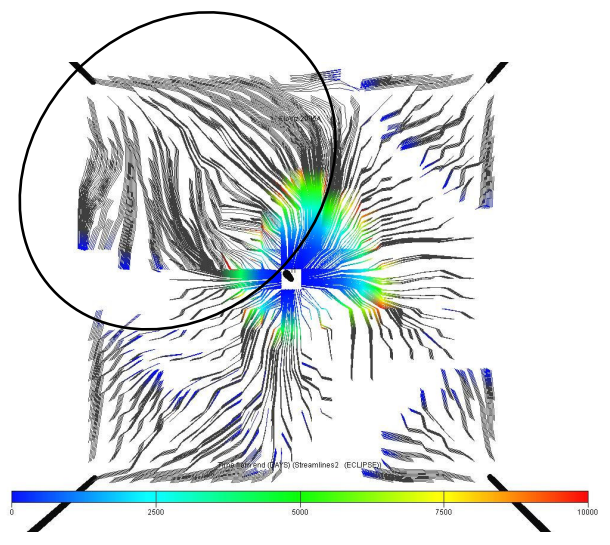

Conventional Run

Figure 13 - Time of Flight Profile for Total Velocity Tracing at $1^{\text {st }}$ Report Step 


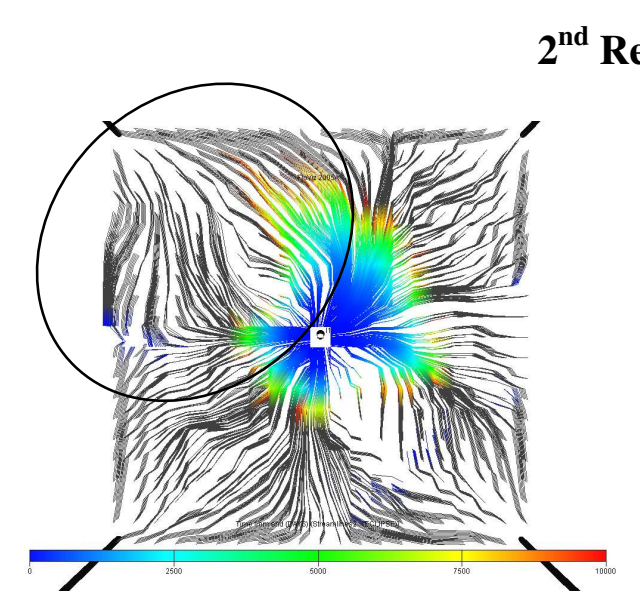

Geomechanics Run $2^{\text {nd }}$ Report-Step

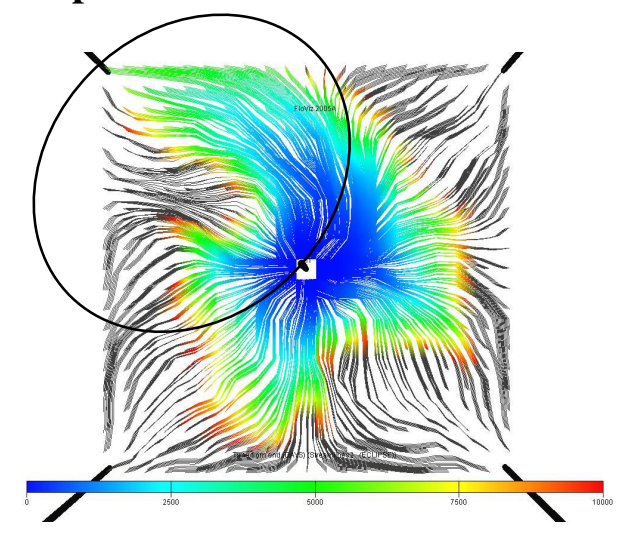

Conventional Run

Figure 14 - Time of Flight Profile for Total Velocity Tracing at $2^{\text {nd }}$ Report Step

It has been said earlier that pore volume change in geomechanics run reduces the rate of pore pressure change which in turn causes low flow potential. For the $1^{\text {st }}$ report step, the maximum pressure in the field for geomechanics run is $4200 \mathrm{psi}$ and minimum pressure is $3950 \mathrm{psi}$ whereas for conventional run the maximum and minimum pressures are 5200 psi and 3400 psi. Clearly the comparison shows that for geomechanics run, the regions of reservoir that experience pressure rise have low value as compared to same regions for conventional run and similarly regions which experience pressure depletion have higher pressure due to support by pore volume reduction. The impact of this pressure variation is visible on the traced streamline. The time of flight profiles for both simulations are compared in the figure above at early stages of reservoir production. For the $1^{\text {st }}$ report step, streamlines tracing for geomechanics run suggests that flow is taking place near well regions only and the rest part of the reservoir is not contributing to the flow. As a result the oil production rates for the producers are higher for conventional 
run. For $2^{\text {nd }}$ report step, color scaling clearly tells that time of flight along streamlines in case of conventional run is small and as a result flow is moving fast.

\subsubsection{Geomechanics Impact on Flow Direction}

The way in which geomechanics has influenced the oil production for P1 well is an important issue which needs to be addressed. Production for geomechanics run lags behind production for conventional run for early report steps but it is higher at later report steps. The increase in oil rate for geomechanics run is due to different flow direction and improvement in permeability for region between P1 and injector I1. In the top-left quadrant i.e. region between producer P1 and injector I1, flow direction is towards producer P1 for geomechanics run and remains as such till $7^{\text {th }}$ report step but in conventional run, flow direction changes with each report step and from $3^{\text {rd }}$ report-step the direction stabilizes.

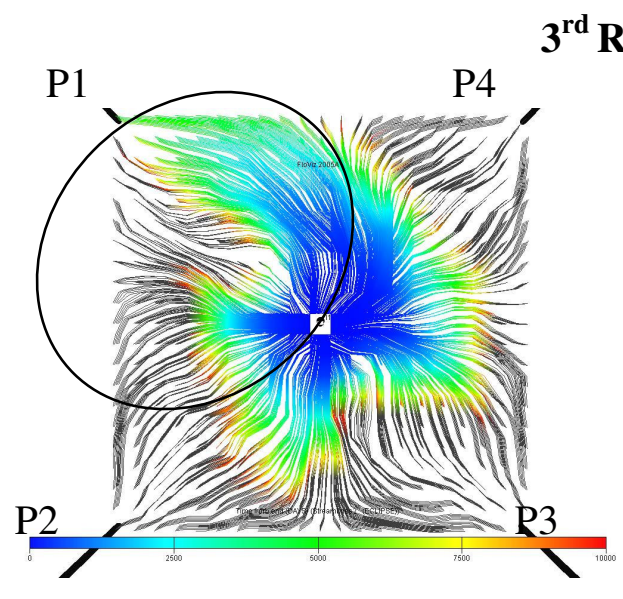

Geomechanics Run $3^{\text {rd }}$ Report-Step

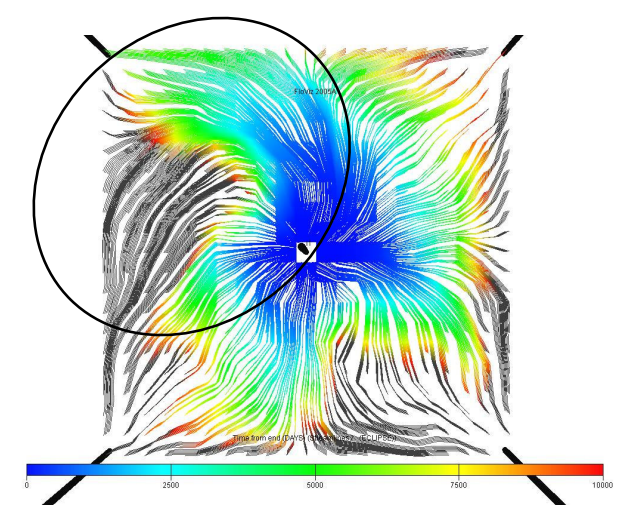

Conventional Run

Figure 15 - Time of Flight Profile for Total Velocity Tracing at $3^{\text {rd }}$ Report Step 


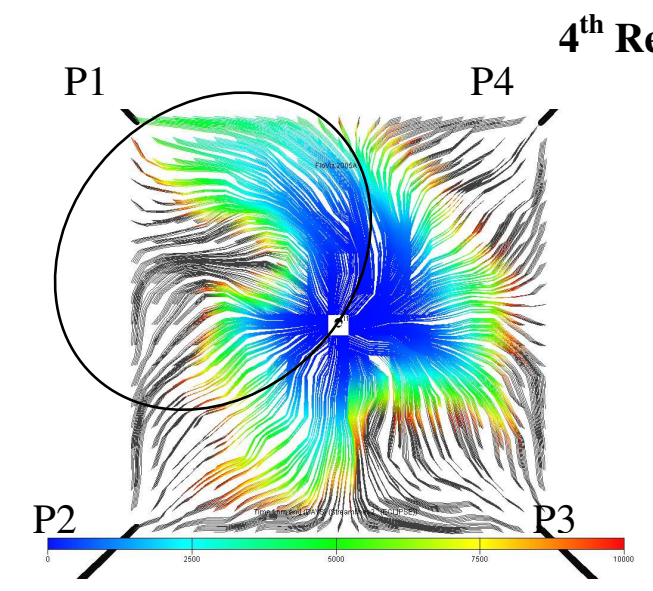

Geomechanics Run

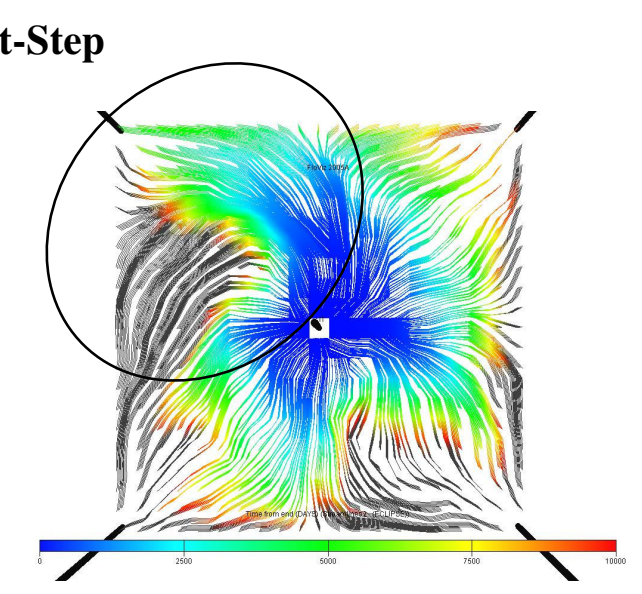

Conventional Run

Figure 16 - Time of Flight Profile for Total Velocity Tracing at $4^{\text {th }}$ Report Step

The flow direction is changing in geomechanics run but change is slow and from $7^{\text {th }}$ report step, the flow profile in geomechanics run stabilizes and becomes similar to flow profile for conventional run. So, it takes around 270 days for flow to change direction in conventional run whereas it takes around 1300 days for the same effect in geomechanics run.

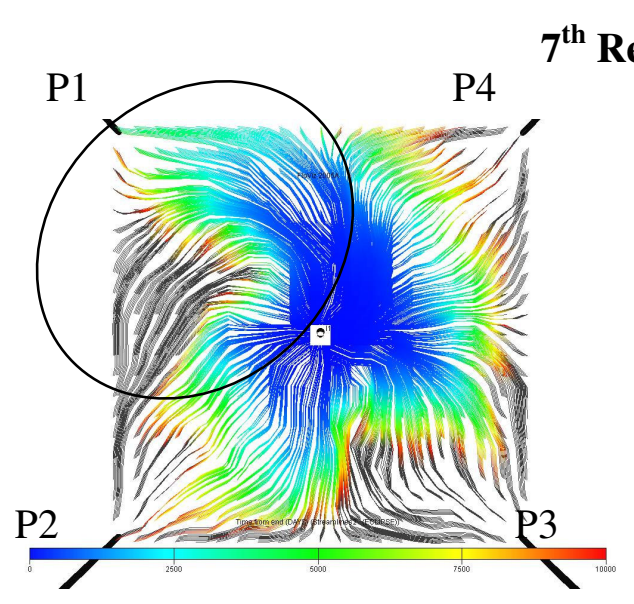

Geomechanics Run

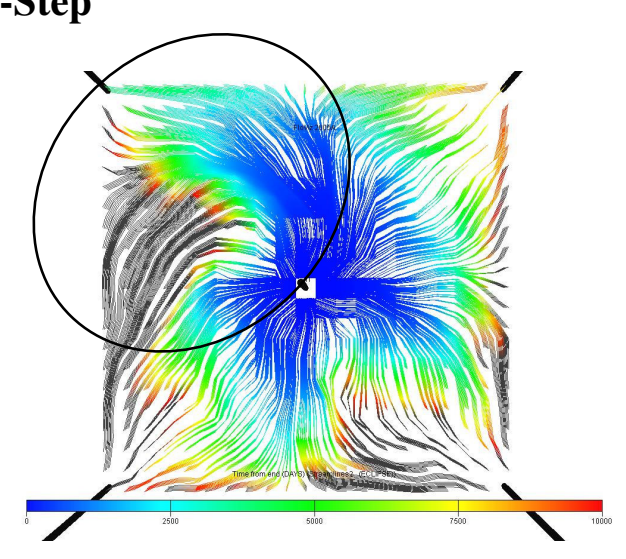

Conventional Run

Figure 17 - Time of Flight Profile for Total Velocity Tracing at $7^{\text {th }}$ Report Step 


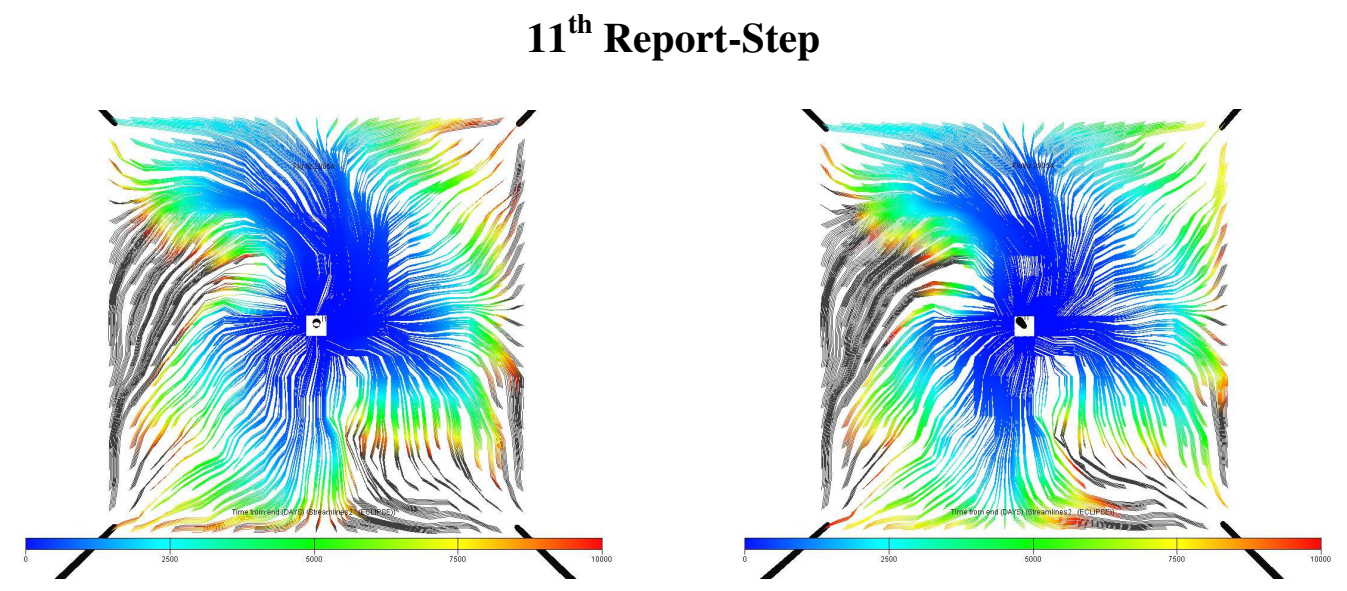

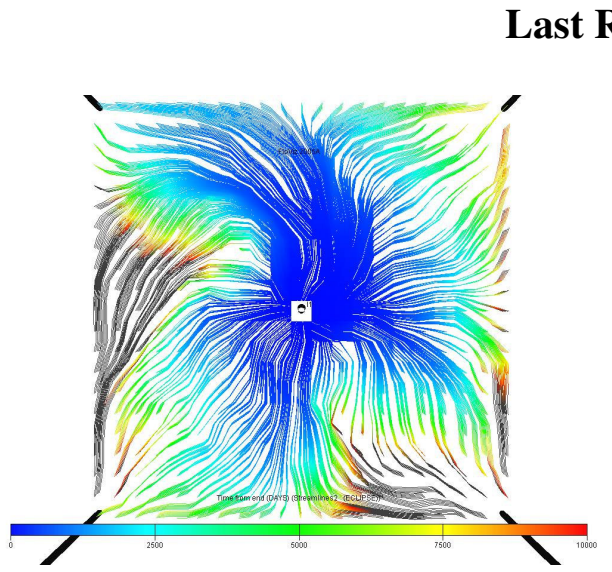

Geomechanics Run

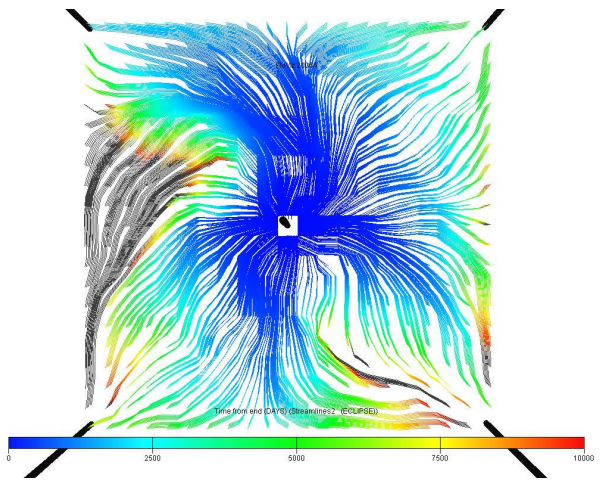

Conventional Run

\section{Figure 18 - Time of Flight Profile for Total Velocity Tracing at $11^{\text {th }}$ Report Step}

In general, identification of flow directionality in the reservoir can lead to number of reservoir management decision like drilling of infill injectors and producers in order to take into account preferred directionality of flow and recover oil from unswept regions of the reservoir. Another useful contribution of visualizing flow pattern through streamlines will be assisted history matching of well performance to honor well production history. The static properties for region with preferred flow direction will directly impact the fluid flow and as such, it can be modified for history matching instead of adjusting rock properties for the entire reservoir. 


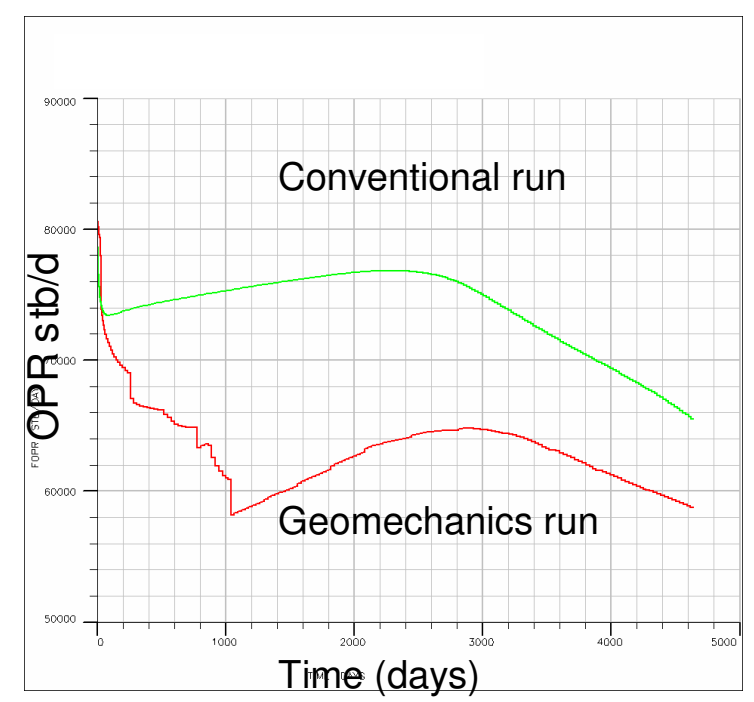

Figure 19 - Plot Showing Field Oil Production Rate for Runs With and Without Geomechanics Option

The average field oil production rate for conventional run is 75,000 BOPD and for geomechanics run is $65,000 \mathrm{BOPD}$. The higher production rate causes oil saturation in the reservoir for the conventional run to decreases more than the oil saturation for the geomechanics run although the difference is not very large.

\section{$3^{\text {rd }}$ Report-Step}
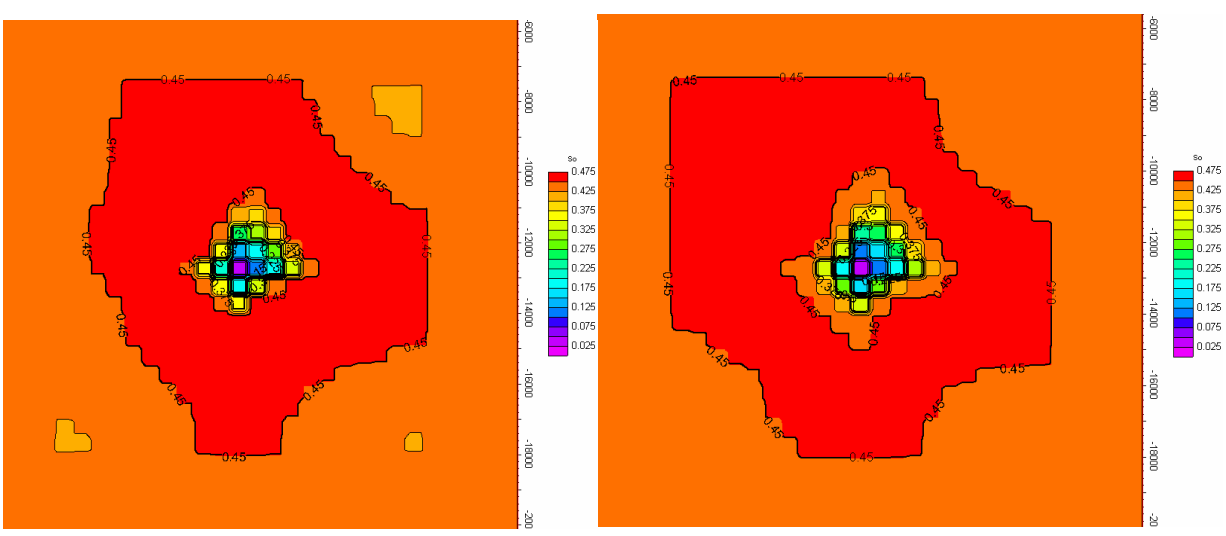

Figure 20 - Oil Saturation Contours at $3^{\text {rd }}$ Report Step 


\section{$10^{\text {th }}$ Report-Step}
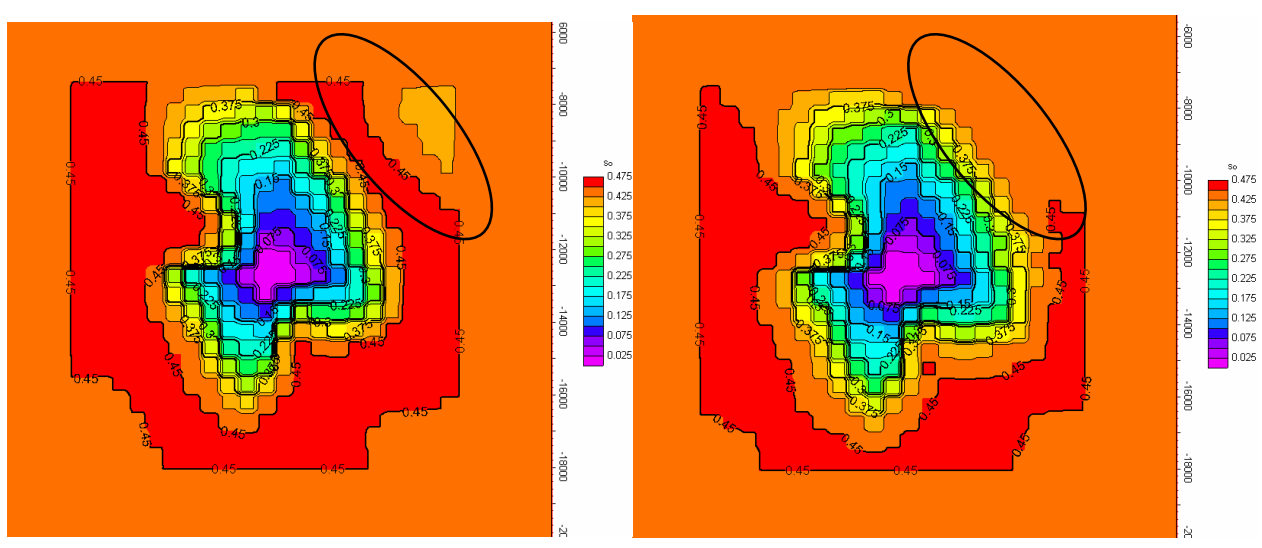

\section{Last Report-Step}

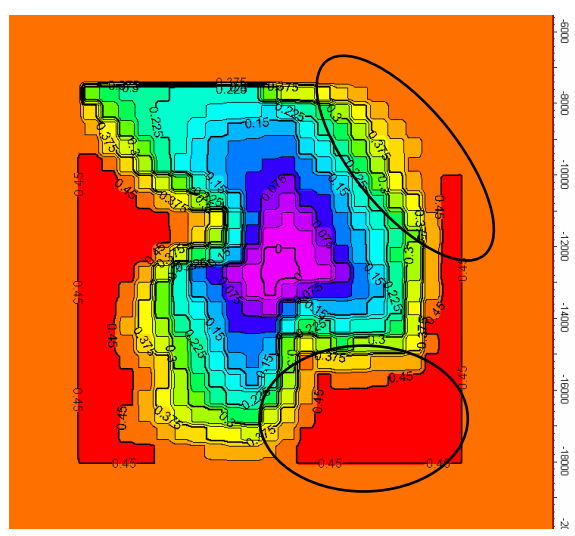

Geomechanics Run

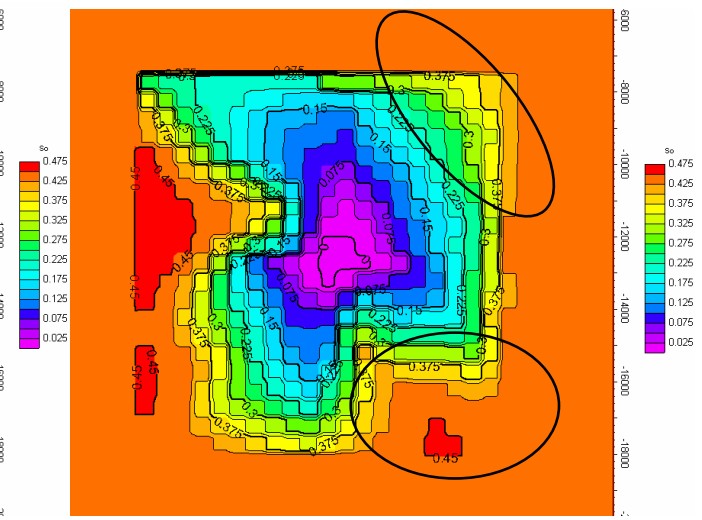

Conventional Run

Figure 21 - Oil Saturation Contours

\subsubsection{Geomechanics Impact on Permeability}

The hydrocarbon production alters pore pressure which in turn changes stresses on reservoir rock. The change in stress state on rock affects properties like porosity and permeability. In ECLIPSE $300 ®$, a table of principal stress versus permeability modifier is used to update permeability values after each geomechanics calculation. 


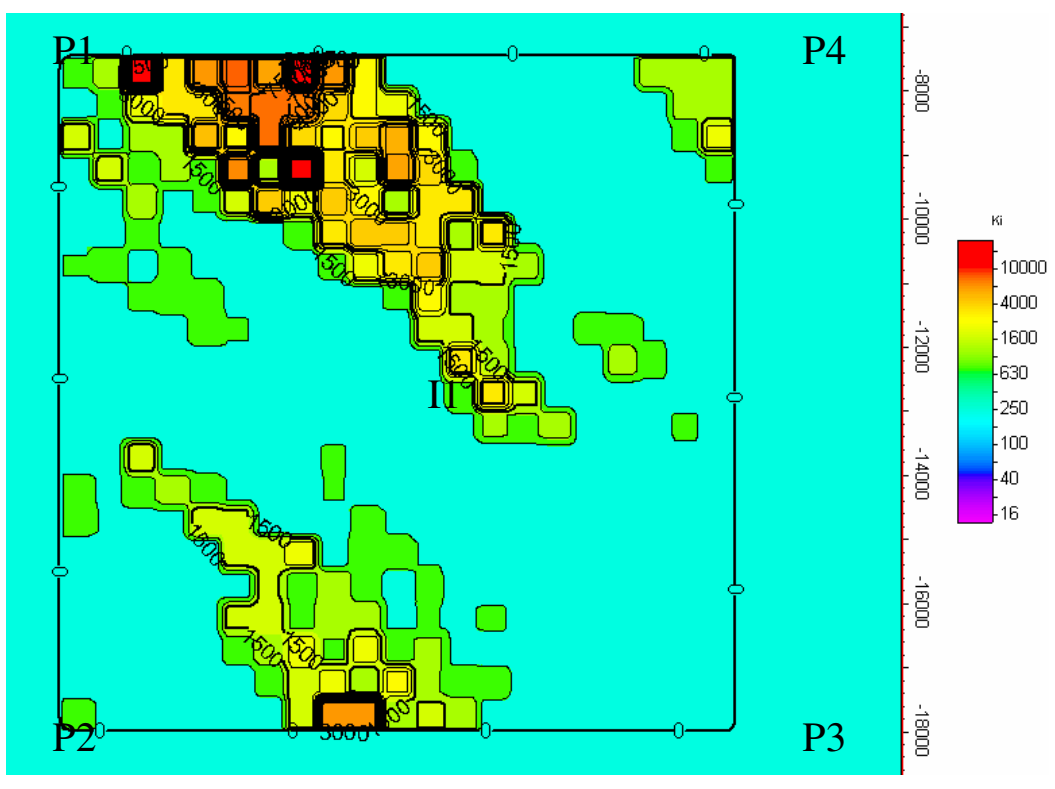

Figure 22 - Contour for Permeability in X-direction for Top Layer

The permeability contours shows that the region between injector I1 and producer P1 has high permeability distribution and the regions towards producers P3 and P4 have low permeability distribution. Due to this permeability variation, waterflood will move faster in region of high permeability i.e. in the direction of producer P1 whereas it will move slowly towards producer P3 and P4. The following time of flight picture for water phase streamline depicts variation in speed of flood front. Clearly, the flood front is moving faster in region of high permeability for both the simulations but the flood front is relatively moving faster in conventional run compared to geomechanics run. 


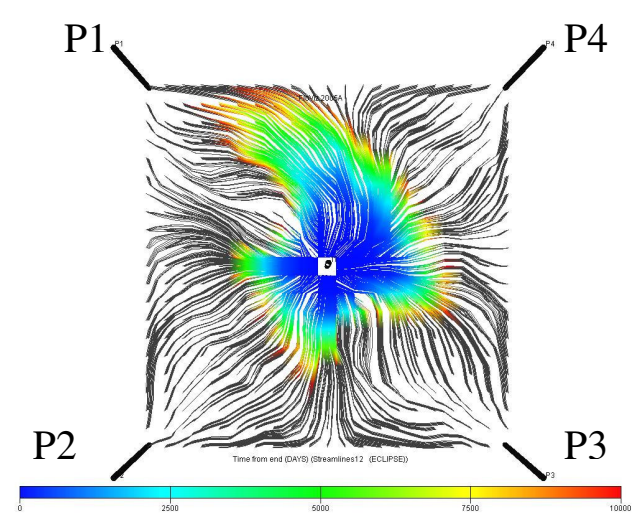

Geomechanics Run

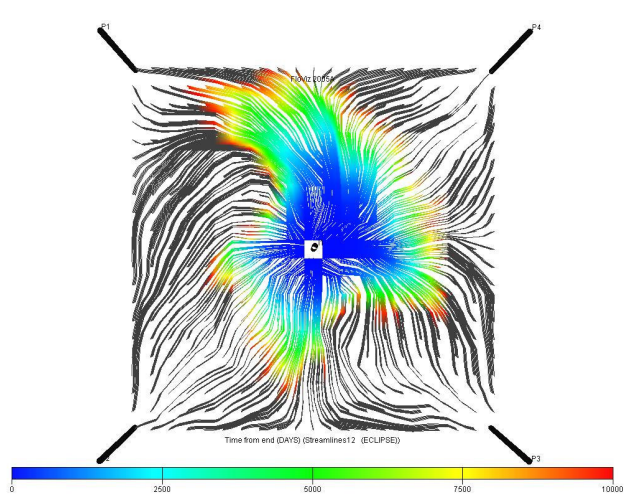

Conventional Run

Figure 23 - Time of Flight Profile for Water Phase Tracing

In geomechanics run, the slow movement of flood front along with pore volume changes will affect pore pressure differently as compared to conventional run. Due to low permeability near producers P3 and P4, there will be delay in arrival of the waterflood and the production of oil will result in pressure depletion in the near well region. The pore pressure for the conventional run will deplete at a faster rate than the geomechanics run because due to reservoir geomechanics, pore pressure depletion will result in increase in effective stresses on the rock which will cause reduction in pore volume and this will support pore pressure. The fast movement of the waterflood in conventional run will cause rise in pore pressure early during the production period compared to very late rise in pore pressure for geomechanics run due to slow movement of waterflood. The plots of pressure contours at various stages during the production are presented below to show impact of geomechanics on pore pressure. 

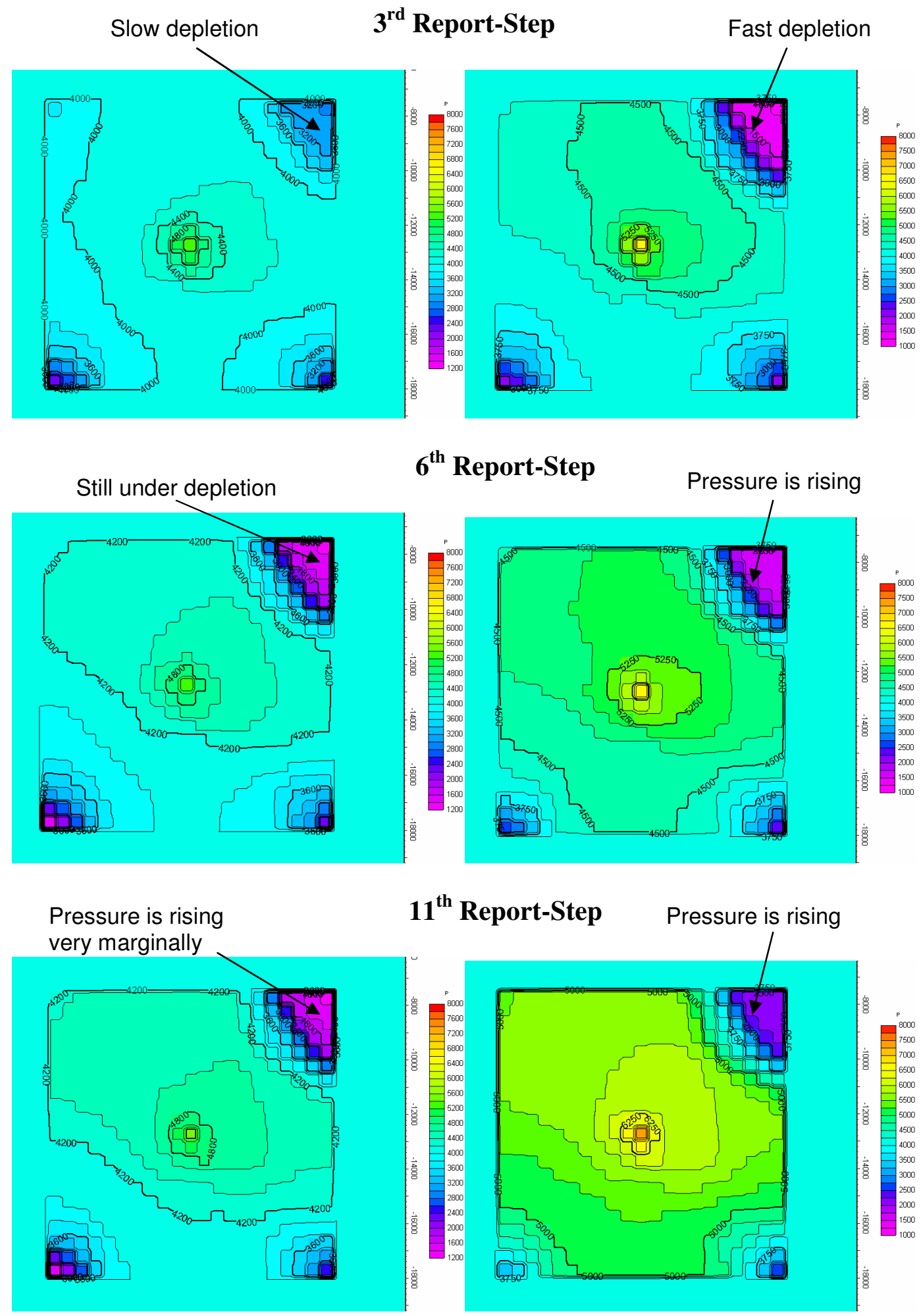

Figure 24 - Pressure Contours 


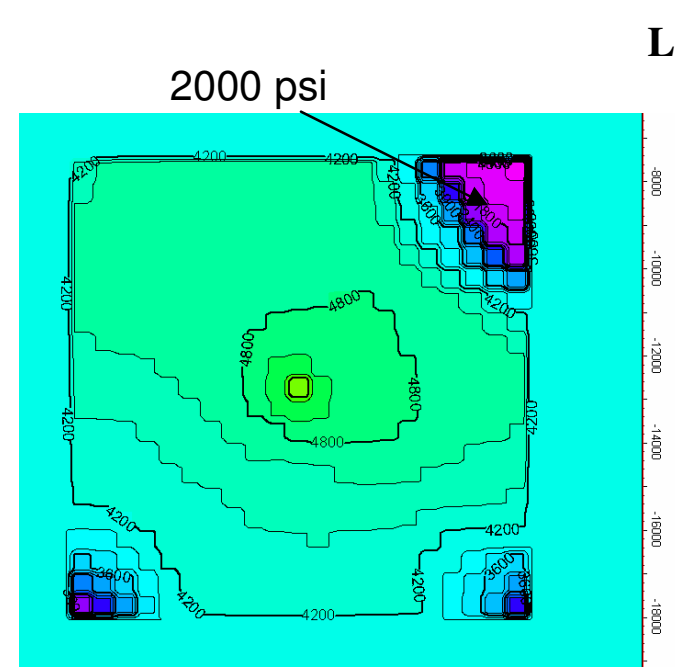

Geomechanics Run
Last Report-Step

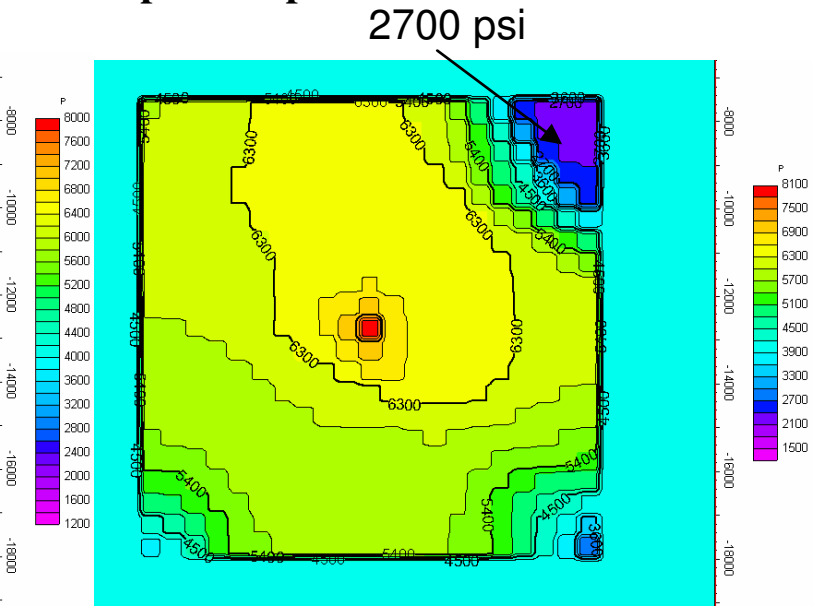

Conventional Run

Figure 25 - Pressure Contours at Last Report Step

Figure 24 highlights the fact that regions with fast movement of waterflood due to high permeability experience early pressure support whereas regions with low permeability experience late pressure support. At the beginning of simulation, pore pressure and overburden stresses on rock are in equilibrium and there is a net effective stress on the reservoir rock. But due to fluid production and fluid injection processes, the pore pressure gets altered which in turn affects the effective stresses on the rock. ECLIPSE $300 ®$ defines relationship of effective stress and pore pressure as

$$
P_{\text {eff }}=P_{\text {fluid }}-\text { Overburden }
$$

At the beginning of simulation, effective stress is same through the reservoir. The regions with pressure depletion witness increase in negative (compressive) effective stresses that causes rock compaction whereas region with rise in pressure witness increase in positive (tensile) effective stress that causes rock to dilate. 


\section{$1^{\text {st }}$ Report Step}

Effective Stress $\mathbf{Z}$ direction

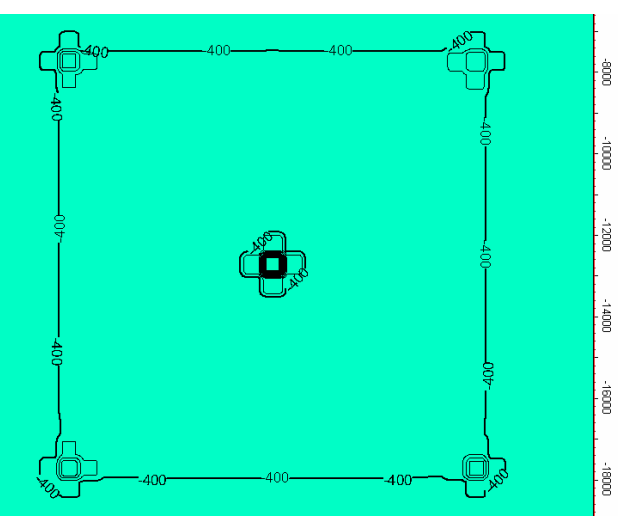

Rock Displacement $\mathbf{Z}$ direction

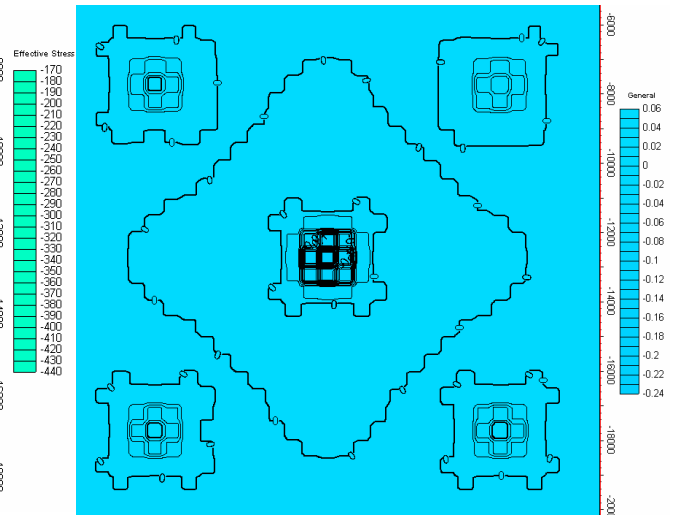

$6^{\text {th }}$ Report Step
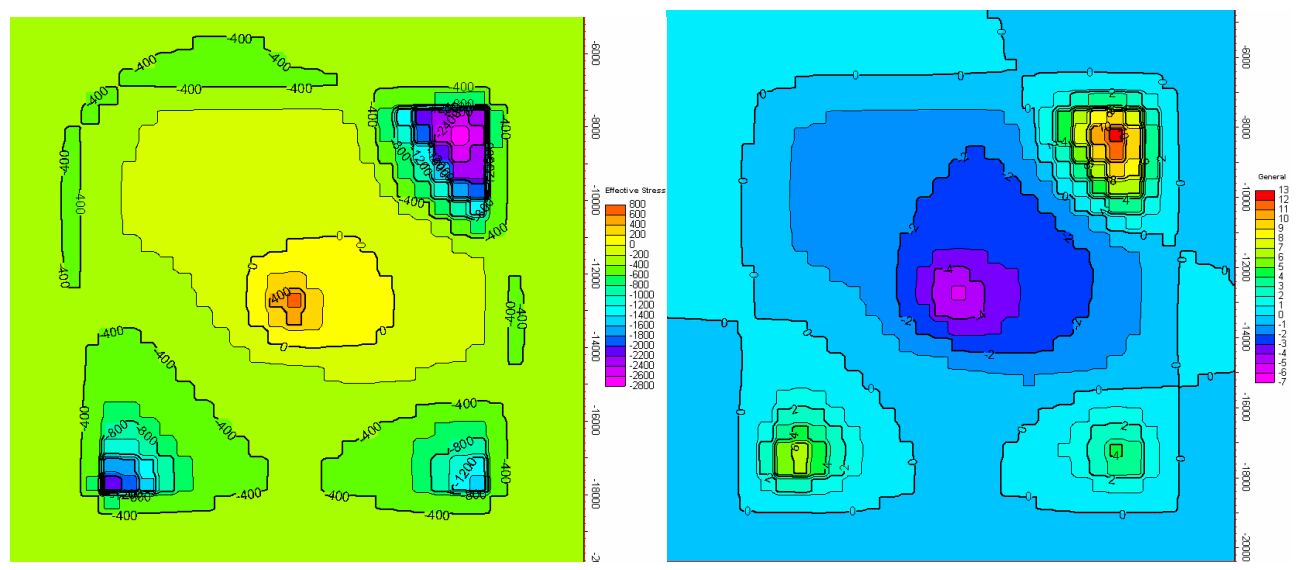

Negative Stress

Last Report Step Rock compaction
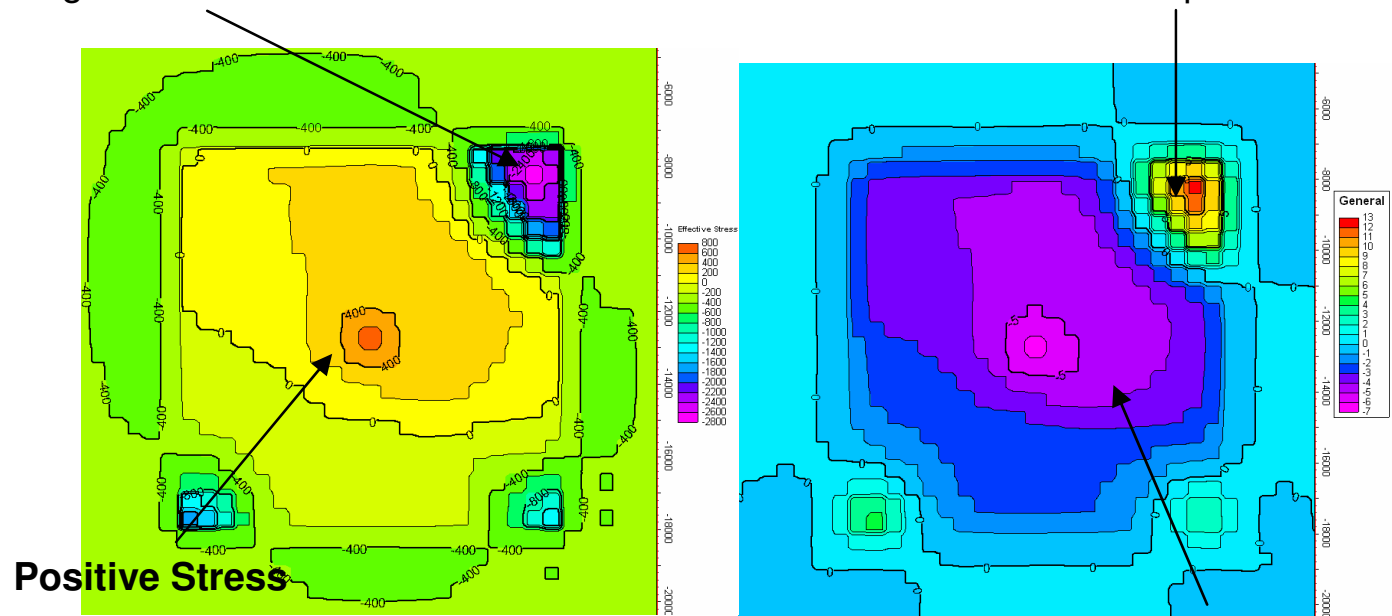

Rock expansion 


\section{$1^{\text {st }}$ Report Step}

Effective Stress Y direction

Rock Displacement Y direction

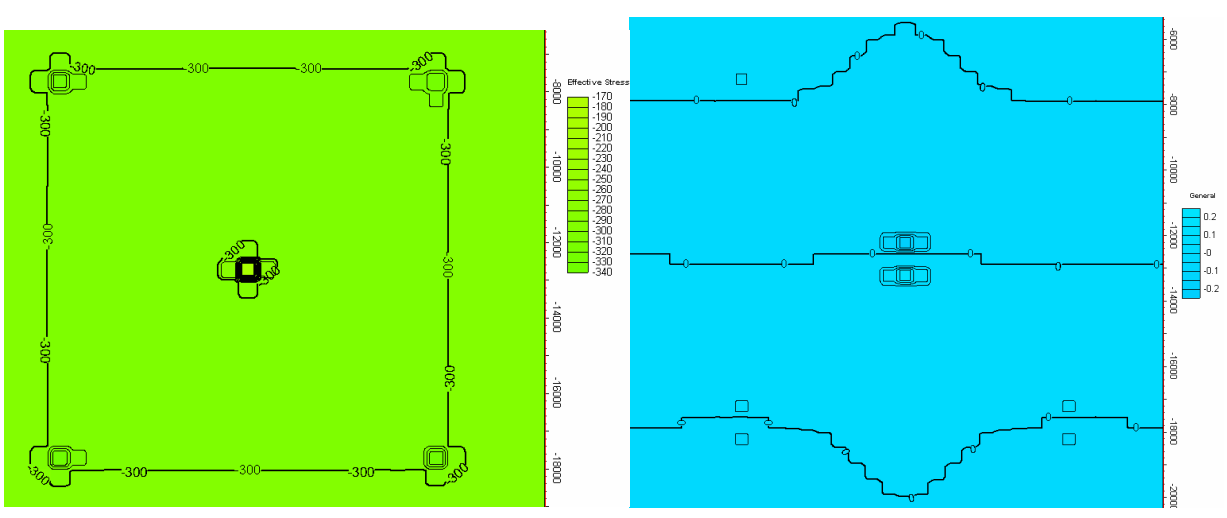

$6^{\text {th }}$ Report Step
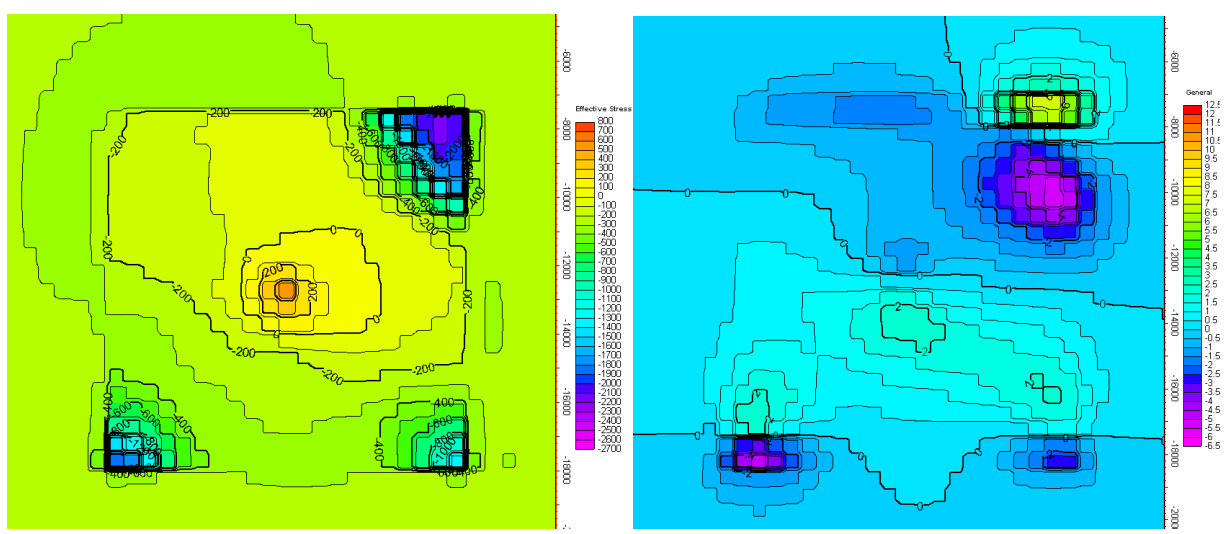

\section{Last Report Step}

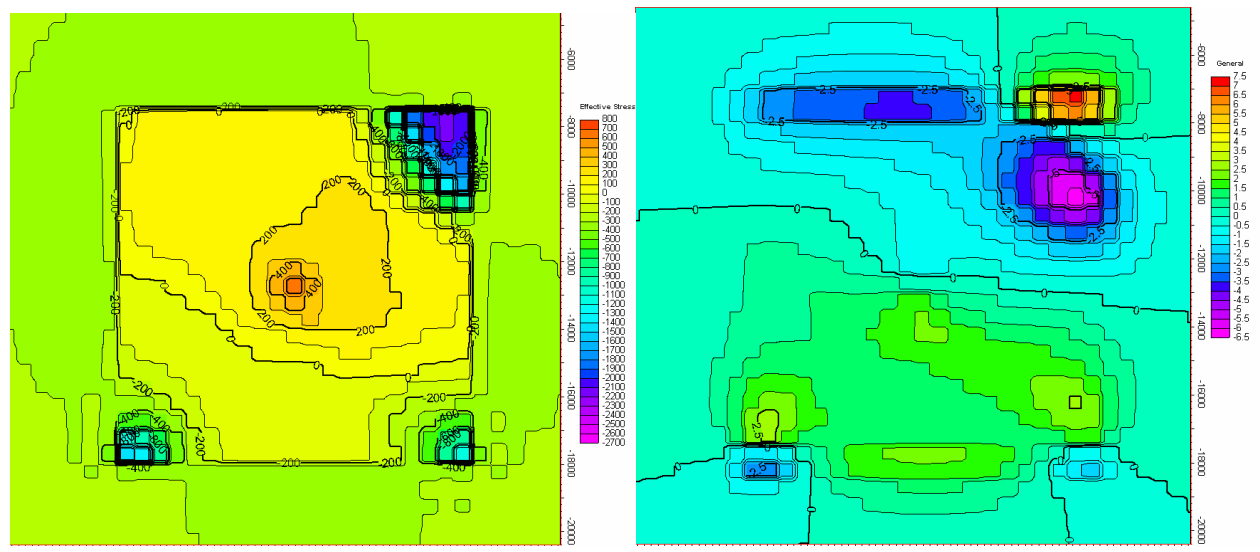

Figure 27 - Effective Stress and Rock Displacement in Y Direction 


\section{$1^{\text {st }}$ Report Step}

Effective Stress X direction

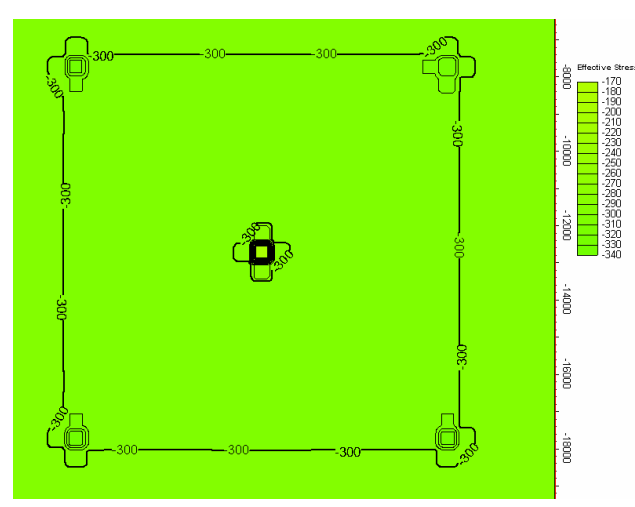

$6^{\text {th }}$ Report Step

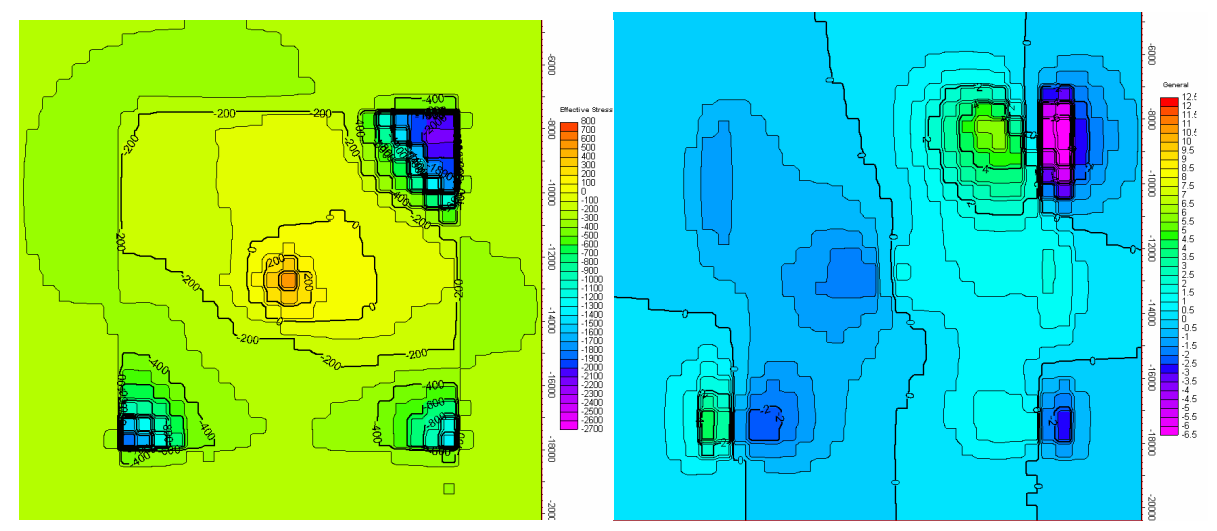

Last Report Step

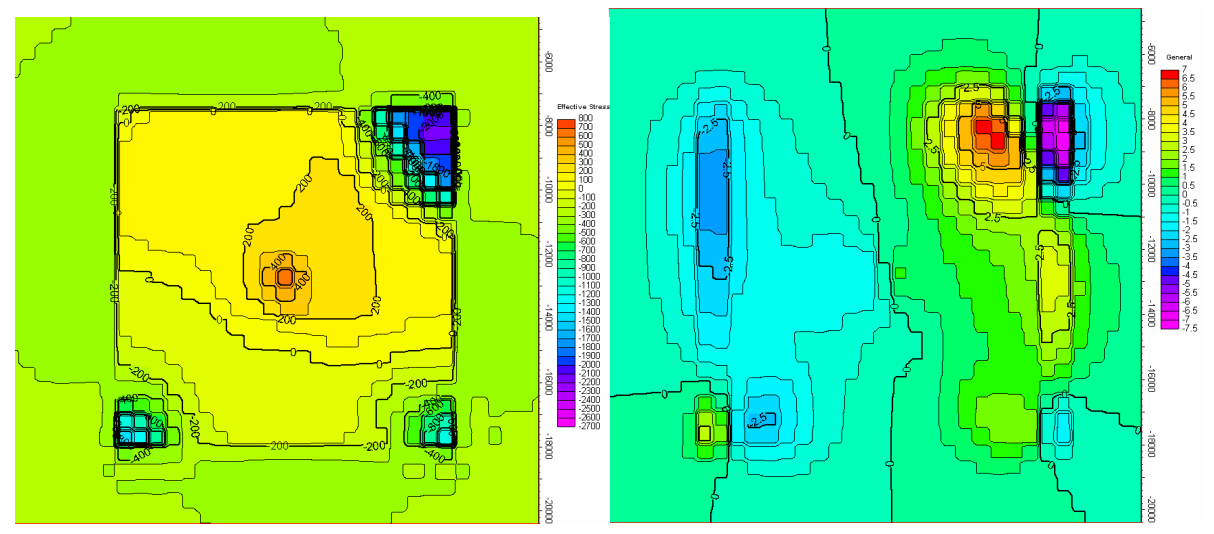

Figure 28 - Effective Stress and Rock Displacement in X Direction 
Figures 25, 26 and 27 for effective stress and rock displacement show how different parts of reservoir respond to geomechanics behavior. The change in fluid pressure also affects the principal stresses acting on the rock. Rock permeability is related to principal stresses so during the course of fluid production there will a change in permeability.

\section{$1^{\text {st }}$ Report Step}

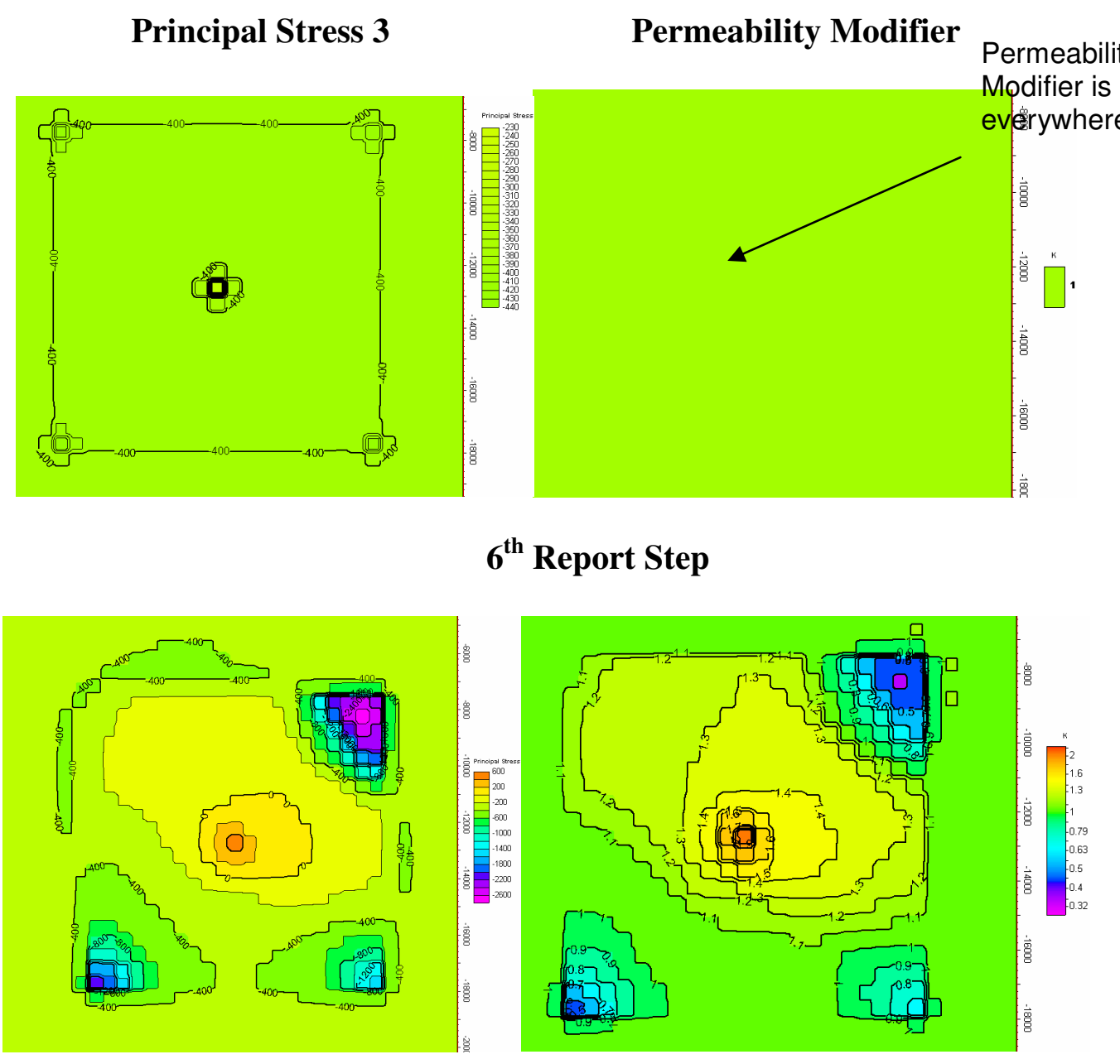

Figure 29 - Principal Stress 3 and Permeability Modifier for Geomechanics Run 


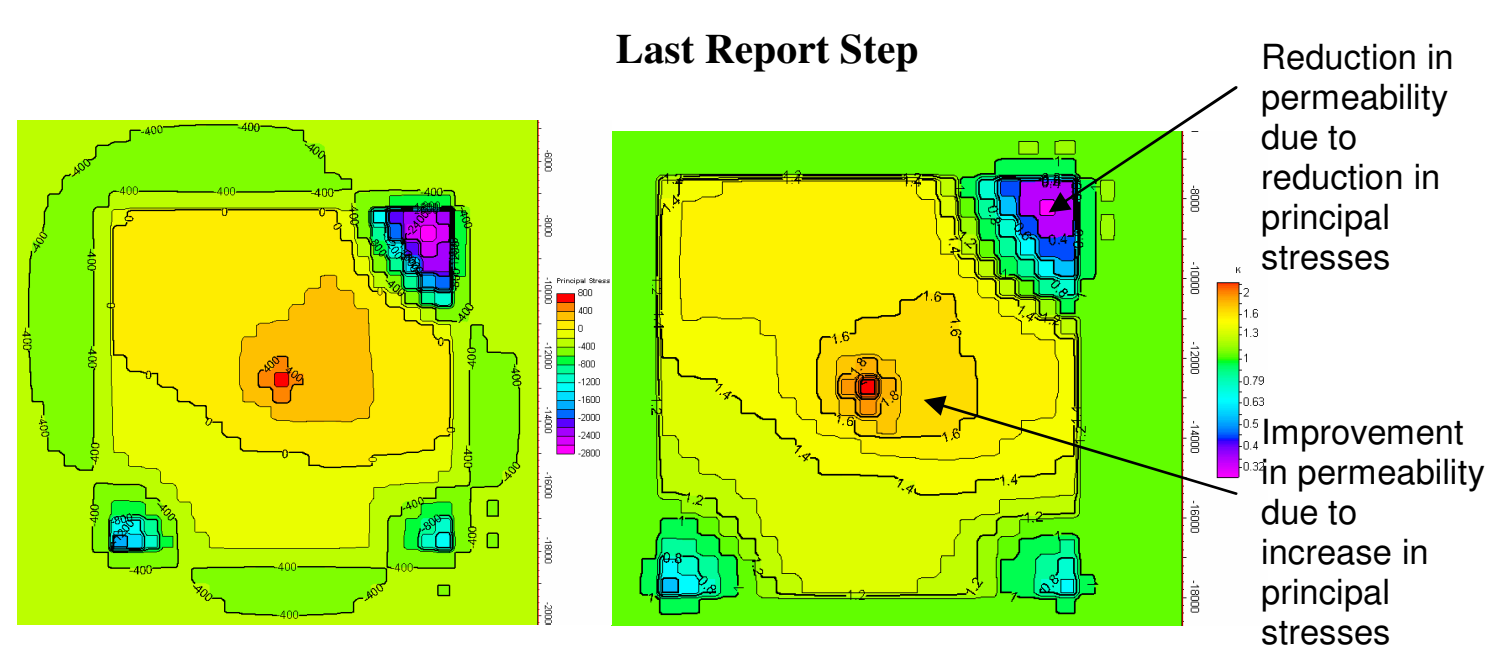

Figure 30 - Principal Stress 3 and Permeability Modifier for Geomechanics Run at Last Report Step

In the conventional run, permeability remains constant for all the time steps. For geomechanics run, the plots of principal stress and permeability modifier shows that regions near producers $\mathrm{P} 1$ and $\mathrm{P} 2$ where there is rise in pressure, see an improvement in permeability and this results in higher oil production for these wells compared to conventional run. Similarly, regions near producers P3 and P4 experience pressure depletion and thus causing a reduction in permeability. This permeability reduction results in oil production to be lower for producers $\mathrm{P} 3$ and $\mathrm{P} 4$ as compared to the same producers for conventional run. 


\subsubsection{Geomechanics Impact on Water Production}

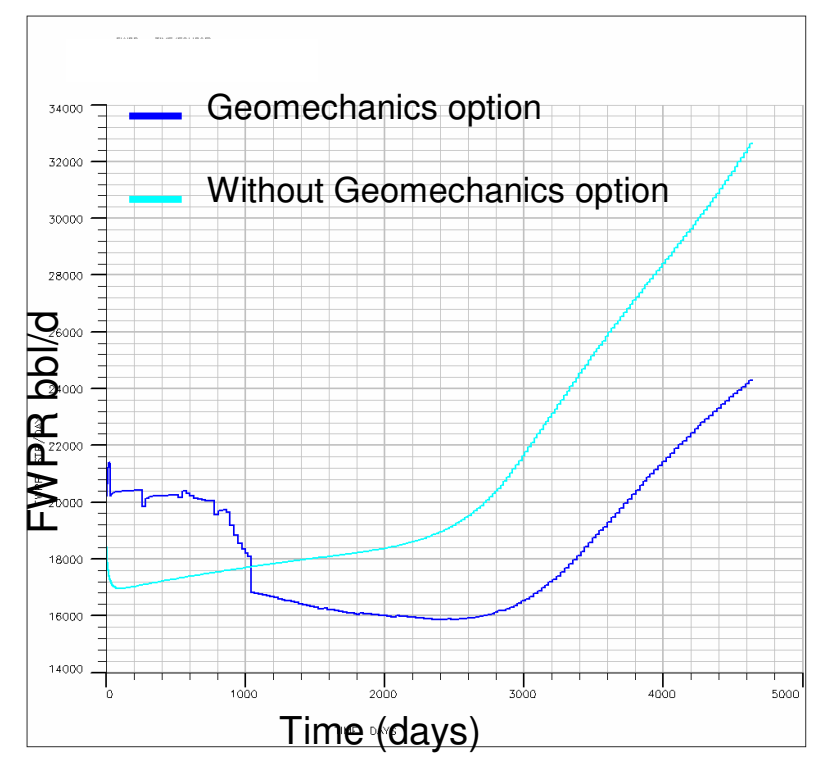

Figure 31 - Field Water Production Rate

Reservoir geomechanics which leads to low flow rates during early period of fluid production also delays the water breakthrough at each of the producers. The plots for water production rates and water cuts for producers have been presented. For both simulations, the producers have been set to constant reservoir volume rate control. At the beginning of simulation, the oil rates for all the producers are lower for geomechanics run compared to conventional run. Because of the rate control method the total fluid production needs to be at constant value and so, as a result the producers have higher water production rate for geomechanics run. But as simulation progresses, conventional run has a rapid movement of flood front leading to early water breakthrough and higher water production rates for producers. 


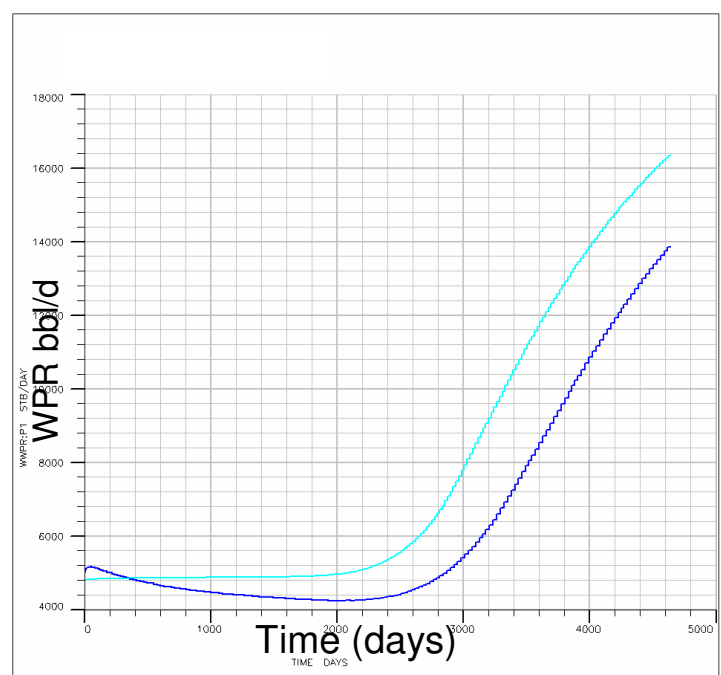

P1 Well

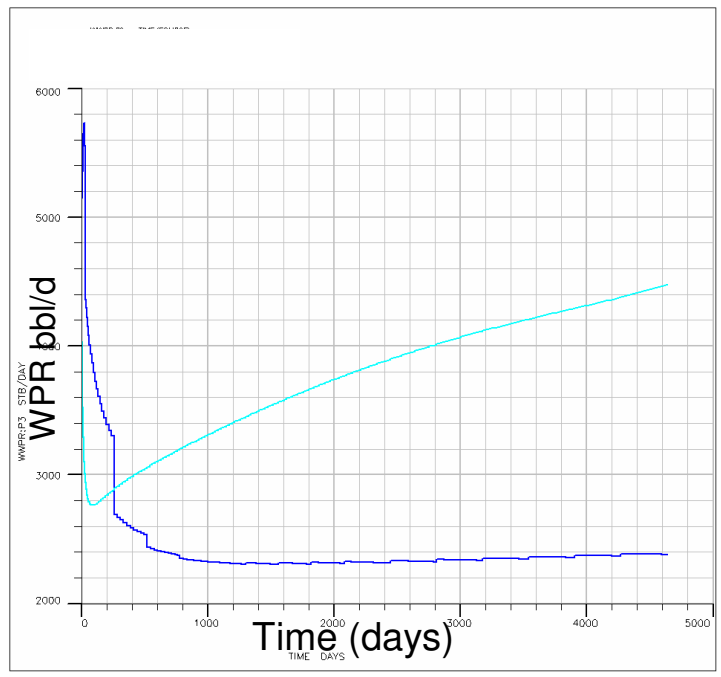

P3 Well

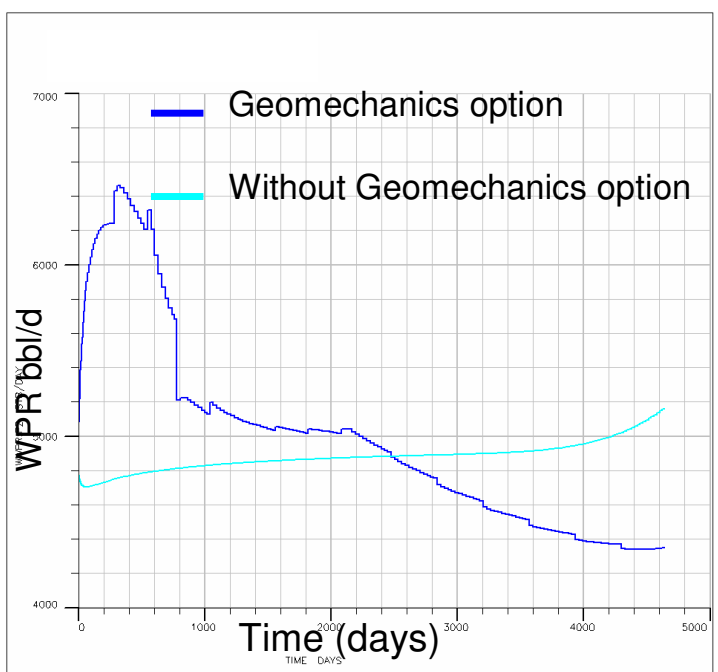

P2 Well

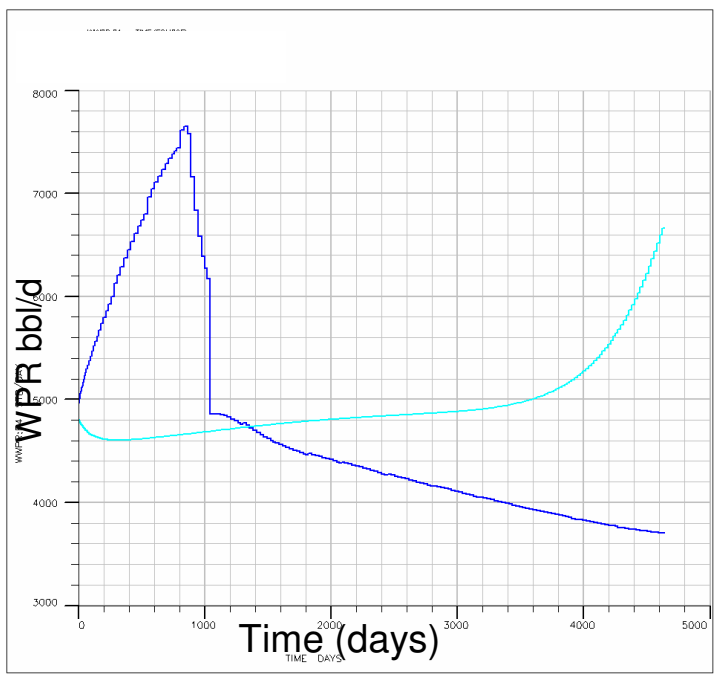

P4 Well

Figure 32 - Plots Showing Water Production Rate for All the Producers

Figure 31 shows that water production for early simulation period is higher for geomechanics run which is opposite to trend of oil rate due to reasons explained above. Figure 32 shows the decrease in water cut for geomechanics run as geomechanics response improves oil rates at the producers and reduces water rate. 


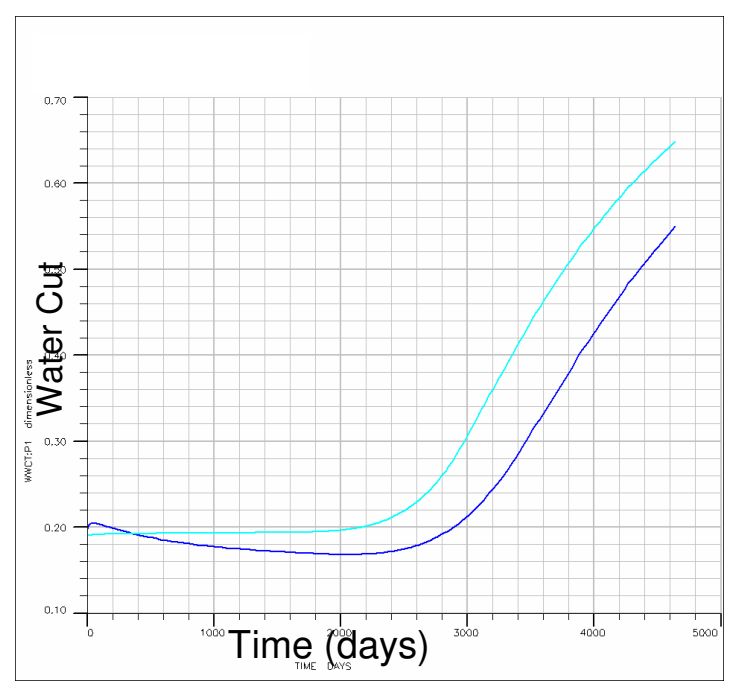

P1 Well

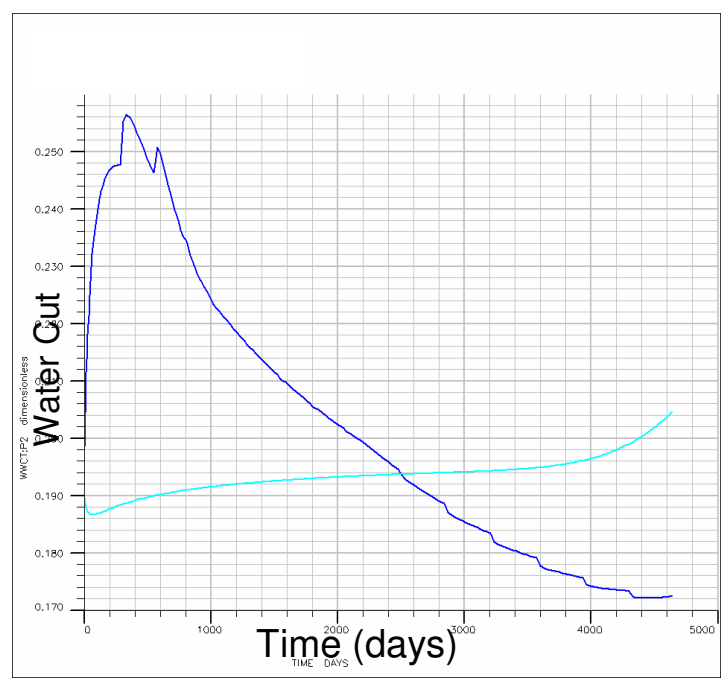

P3 Well

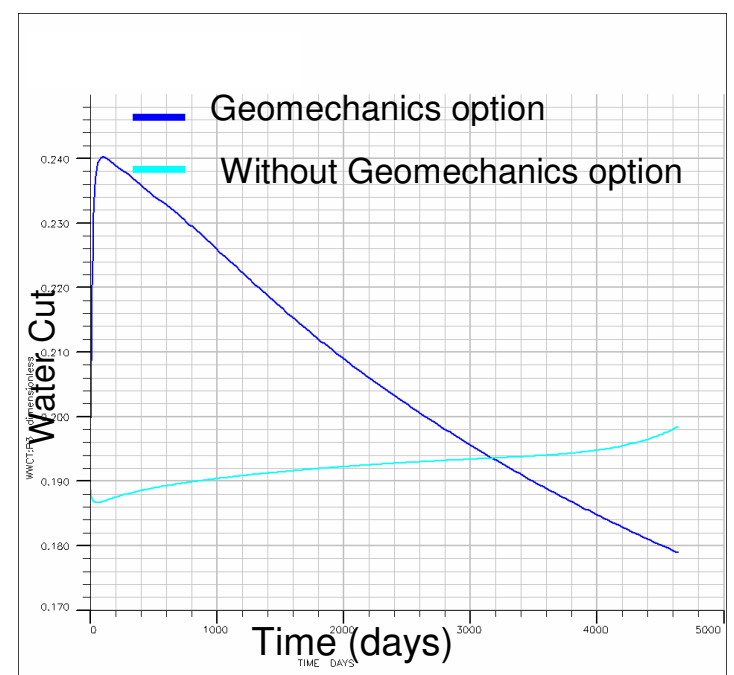

P2 Well

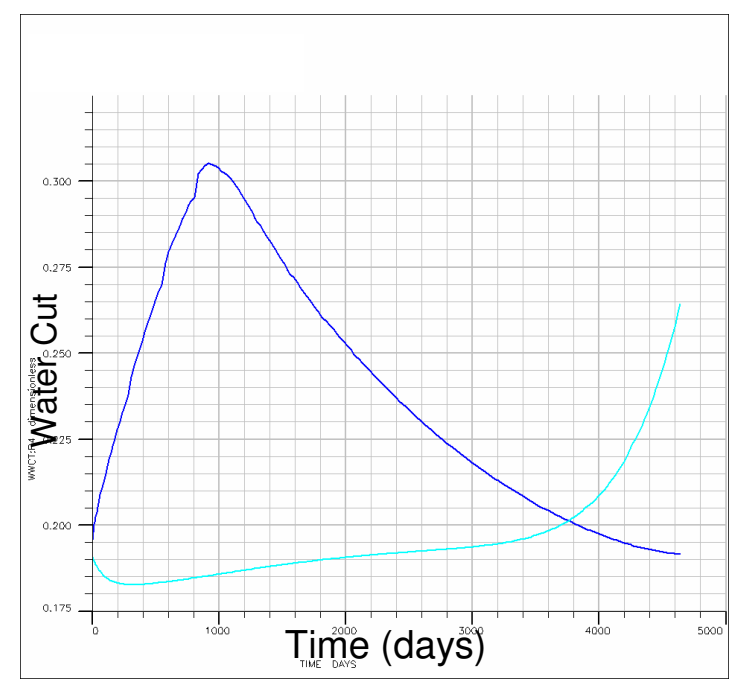

P4 Well

Figure 33 - Plots Showing Water Cut for All the Producers

The time of flight plots for water phase streamline tracing have also been presented. The plots show comparison of water movement through the reservoir. As pointed out earlier about the difference in flood front movement for both the simulation 
runs, it can be seen that time of flight is longer for flow of water in geomechanics run due to slower waterflood movement.

\section{$1^{\text {st }}$ Report-Step}
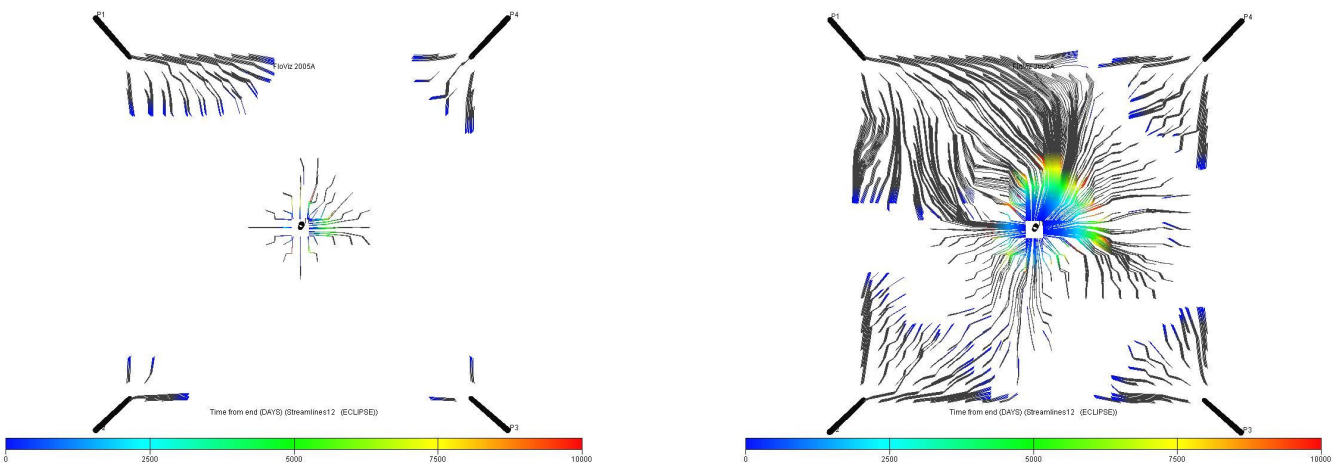

$3^{\text {rd }}$ Report-Step
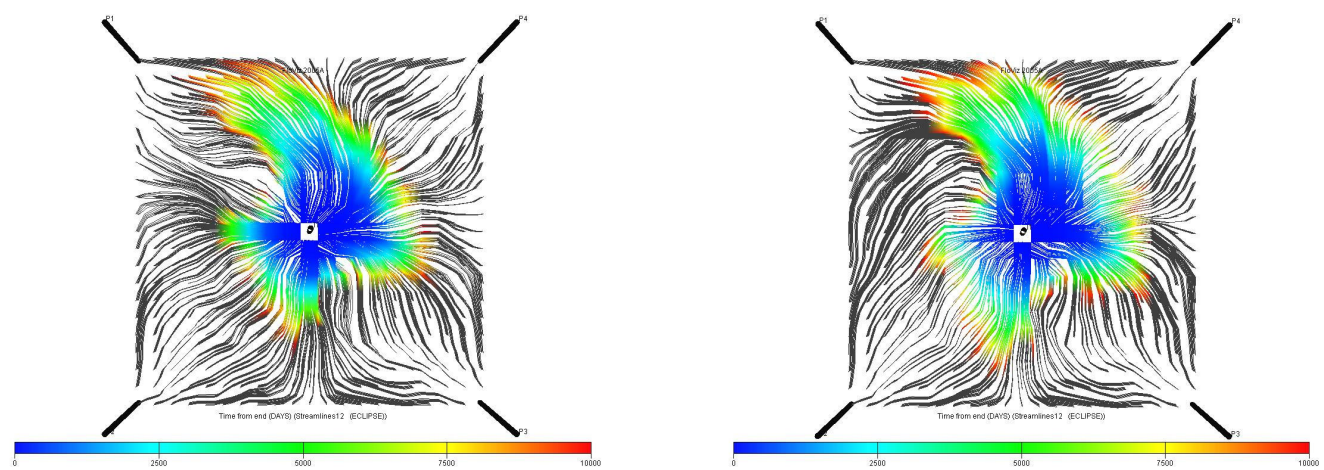

$7^{\text {th }}$ Report-Step

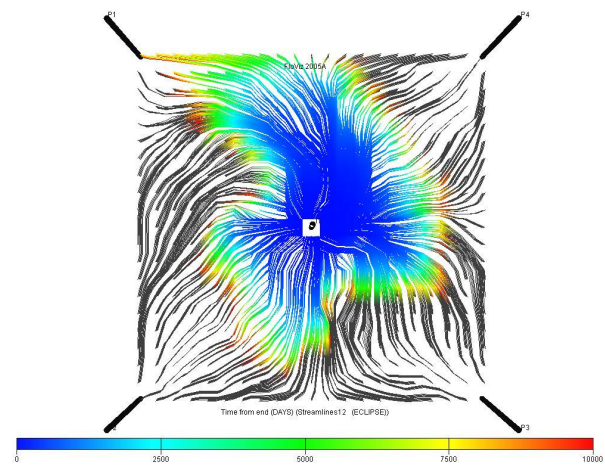

Geomechanics Run

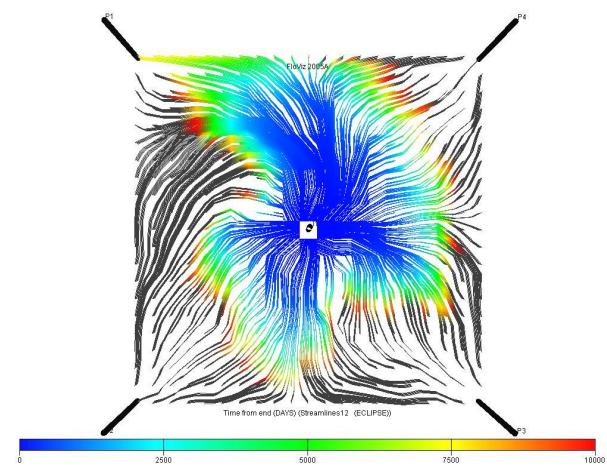

Conventional Run

Figure 34 - Time of Flight Profile for Water Phase Tracing 
$11^{\text {th }}$ Report-Step
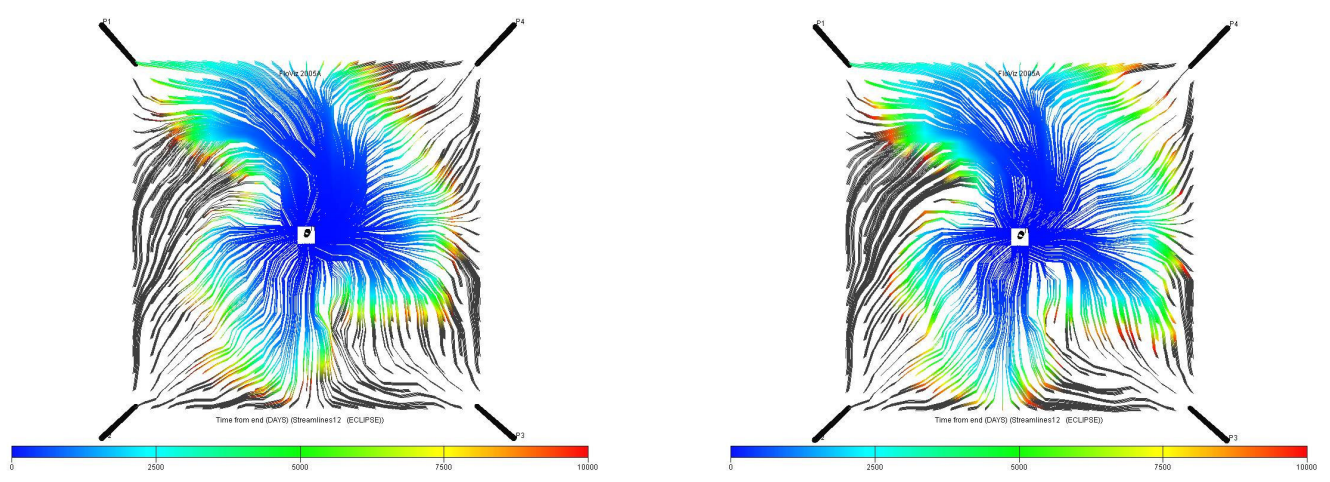

$15^{\text {th }}$ Report-Step
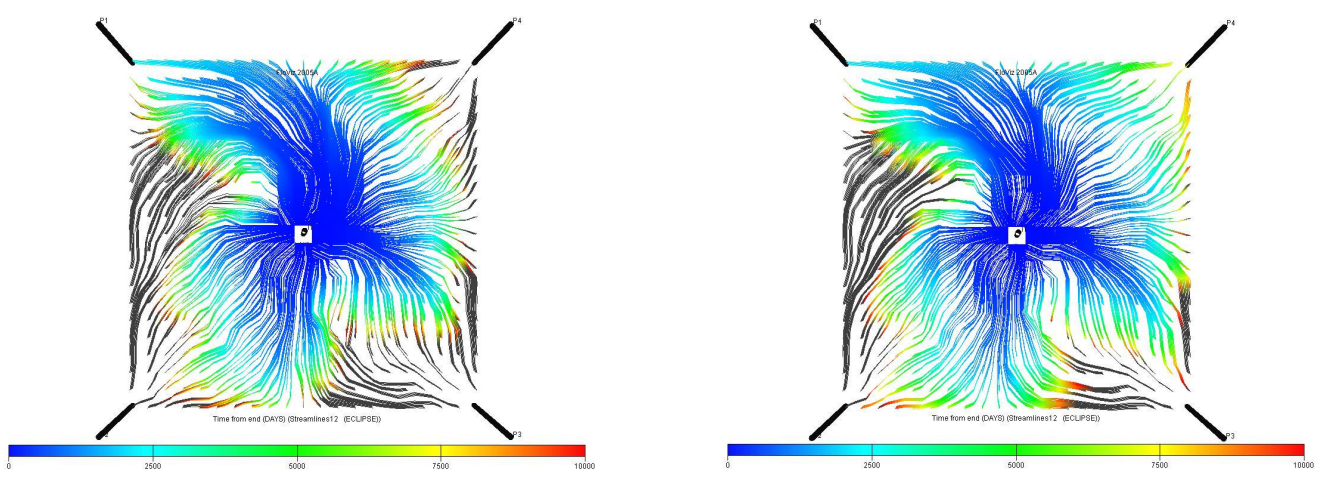

$17^{\text {th }}$ Report-Step

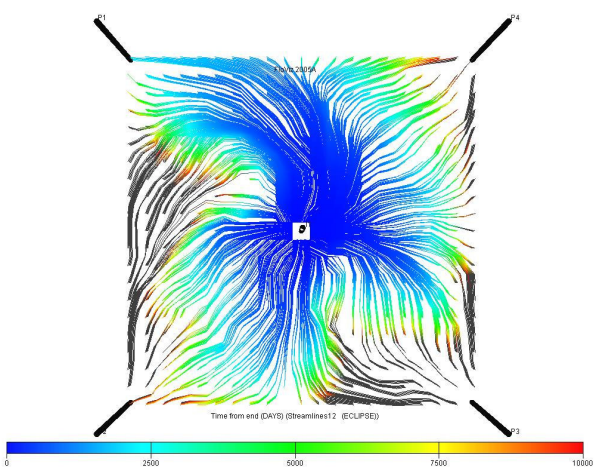

Geomechanics Run

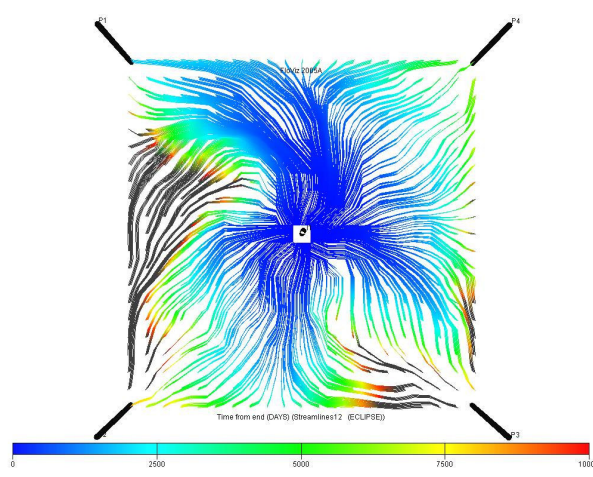

Conventional Run

Figure 35 - Time of Flight Profile for Water Phase Tracing at Different Report Steps 
The water saturation contours follows the same pattern as shown in the time of flight profile for water phase. High flux rate for water phase in conventional run results in greater area being swept by the flood causing increase in water saturation. Also, the profile of contours differentiates region of high permeability from region of low permeability.

\section{$3^{\text {rd }}$ Report-Step}
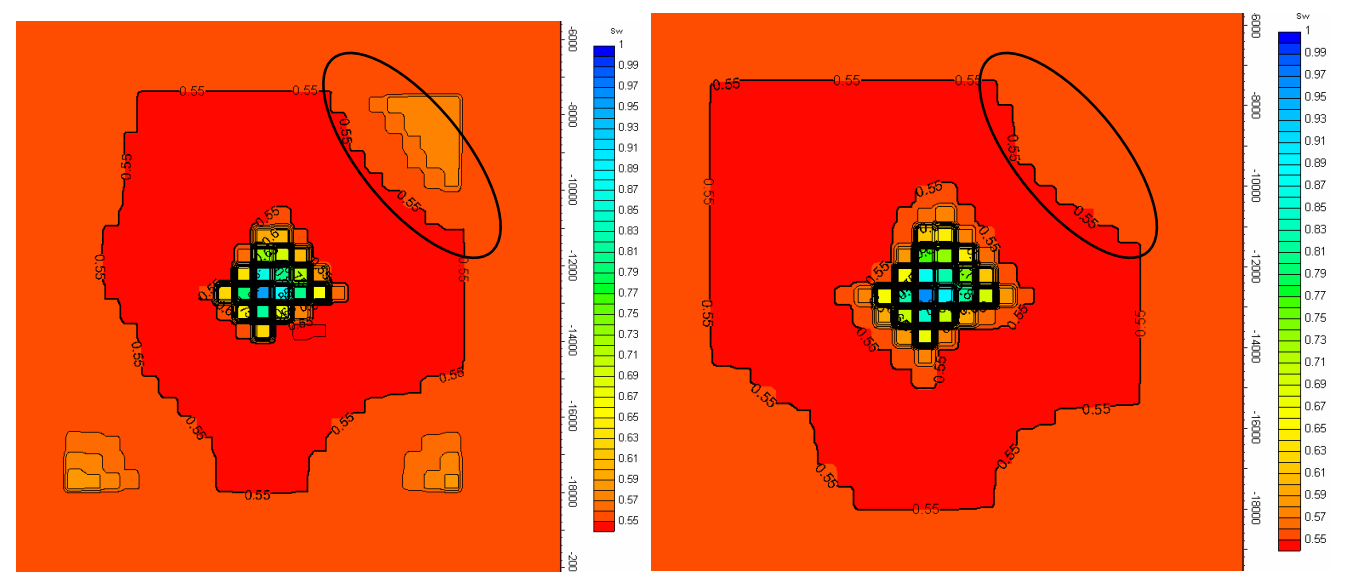

\section{$10^{\text {th }}$ Report-Step}

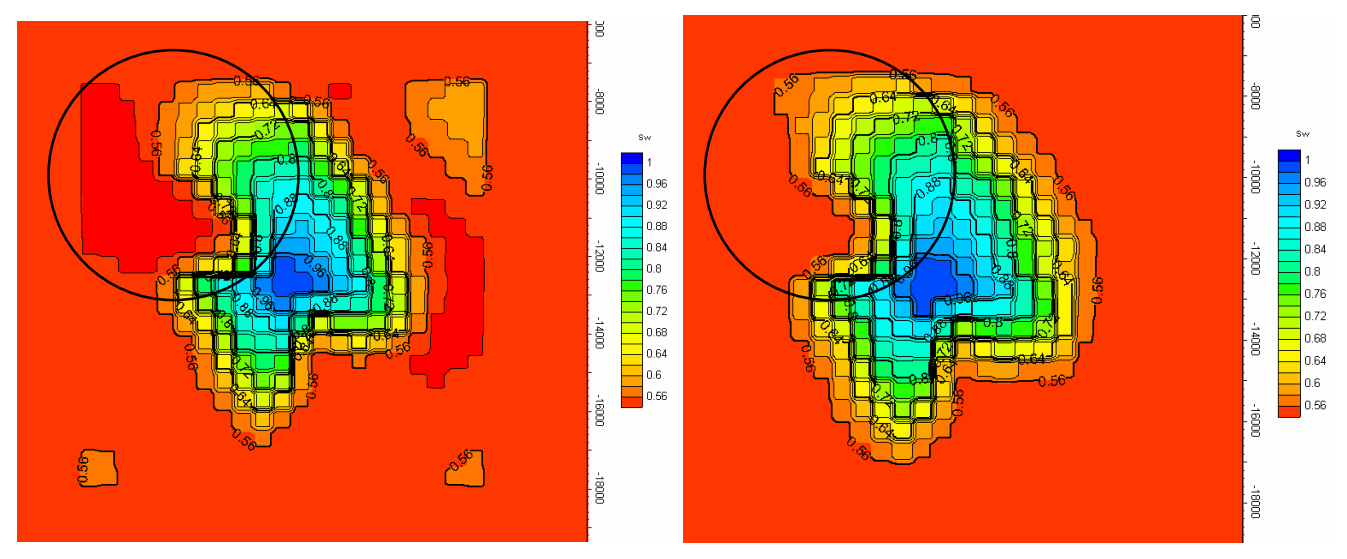

Figure 36 - Water Saturation Contours 


\section{$17^{\text {th }}$ Report-Step}

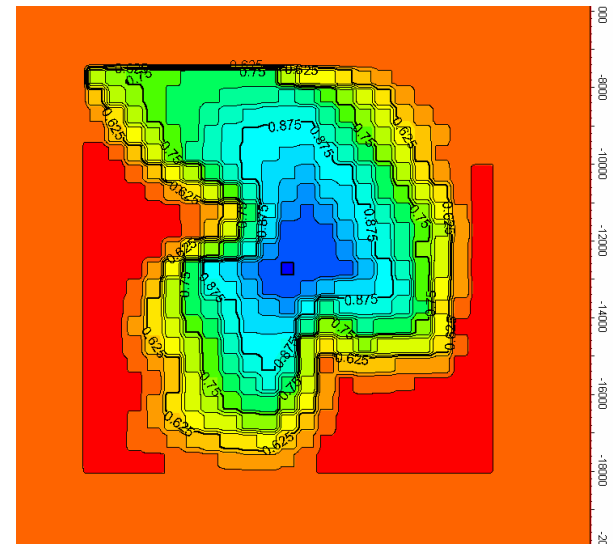

Geomechanics Run

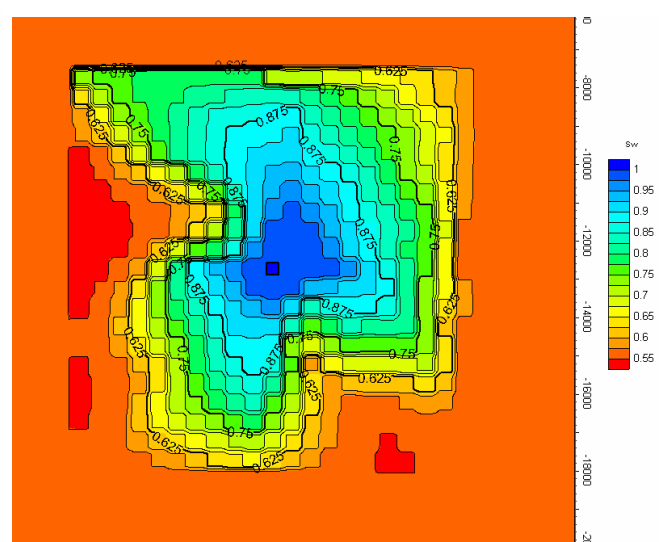

Conventional Run

Figure 37 - Water Saturation Contours at Last Report Step

The heterogeneity of the reservoir influences waterflooding and considering this, the location of injector is not optimum for the present case study. By using water phase streamlines, regions which are not getting affected by waterflooding can be identified and infill injectors can be drilled to improve recovery of oil from unswept regions. 


\subsection{SPE $9^{\text {th }}$ Comparative Study}

The SPE $9^{\text {th }}$ comparative case is a three phase blackoil study with a $3 \mathrm{D}$ heterogeneous grid, 25 producers and one water injection well. Simulation study was conducted and results compared for coupled geomechanics run and conventional run. Original grid of size $24 \times 25 \times 15$ was modified to a size of $34 \times 35 \times 30$ for modeling overburden, side burden and underburden in the reservoir.

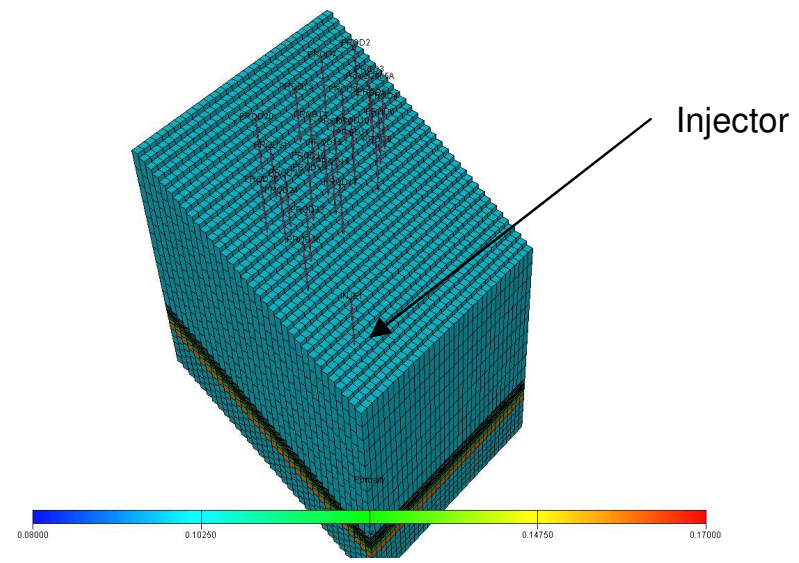

Figure 38 - 3D View of Modified Grid for Geomechanics Study

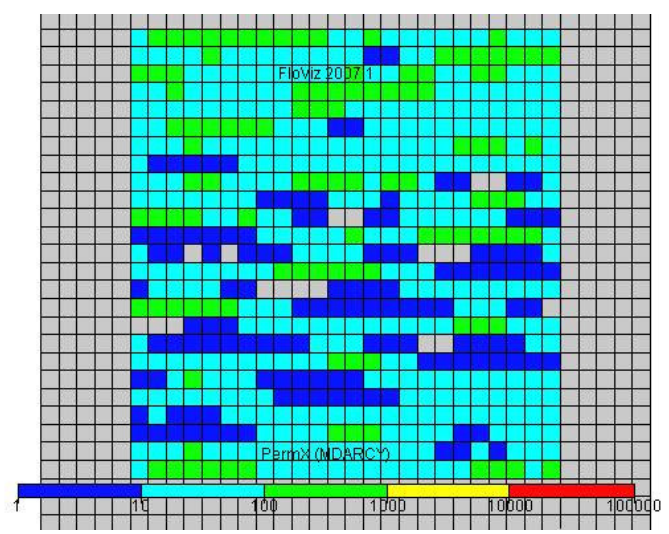

Figure 39 - Top View of Permeability Distribution for the First Reservoir 


\section{Original Grid}

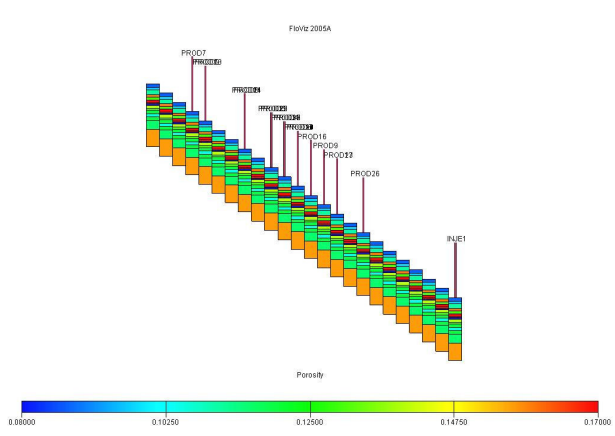

Modified Grid

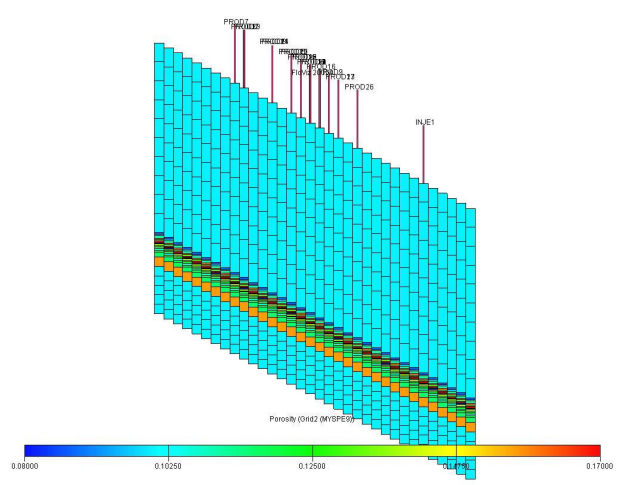

Figure 40 - Cross-Sectional View of Grids

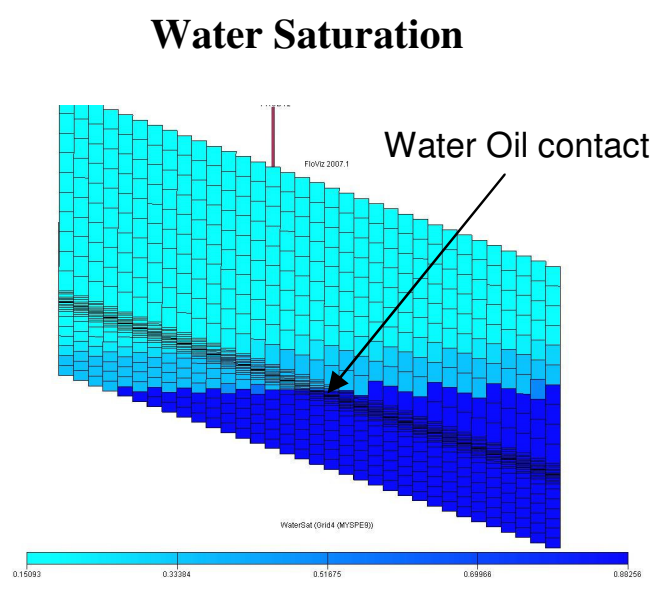

Gas Saturation

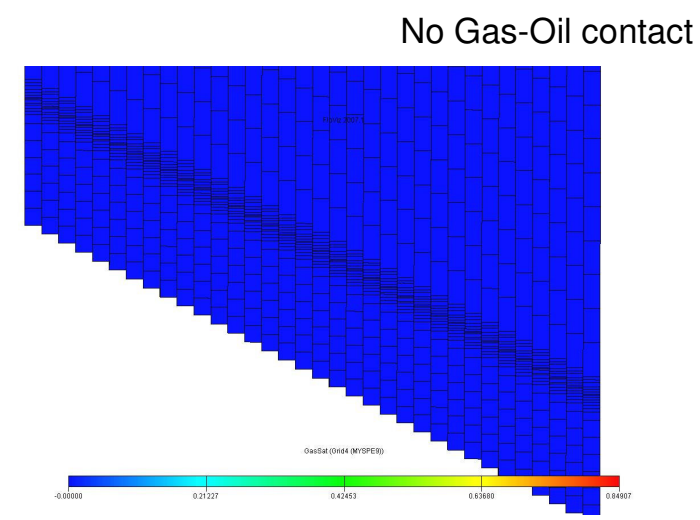

Figure 41 - Fluid Contacts After Initialization of Study

Figure 38 provides the cross-sectional view of original grid and modified grid. Out of 30 layers, 10 layers acts as the overburden and 5 layers act as underburden. Figure 39 shows that at the beginning of the simulation run, gas saturation in the reservoir is zero as there is no gas-oil contact. But due to pressure depletion during the production period, there will be build of gas saturation in the reservoir. 
Field Pressure

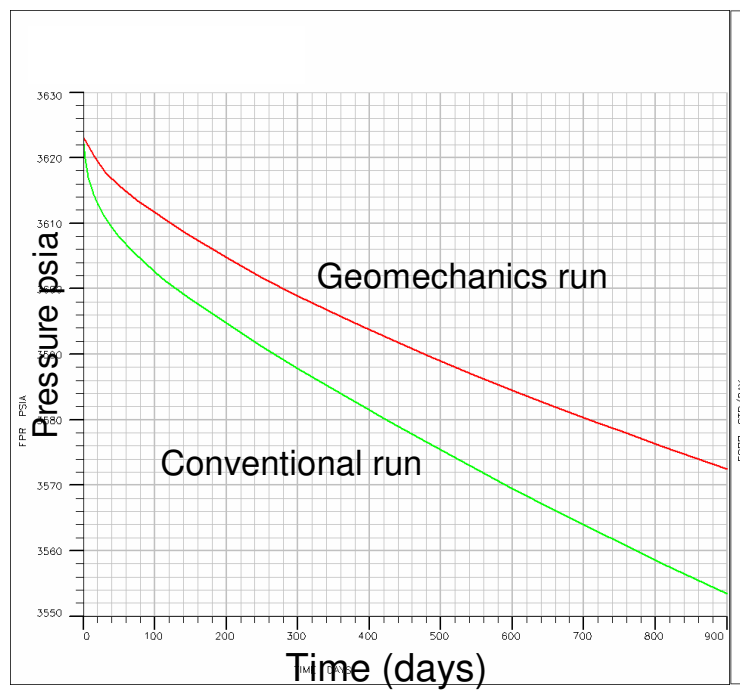

Field Gas Production Rate

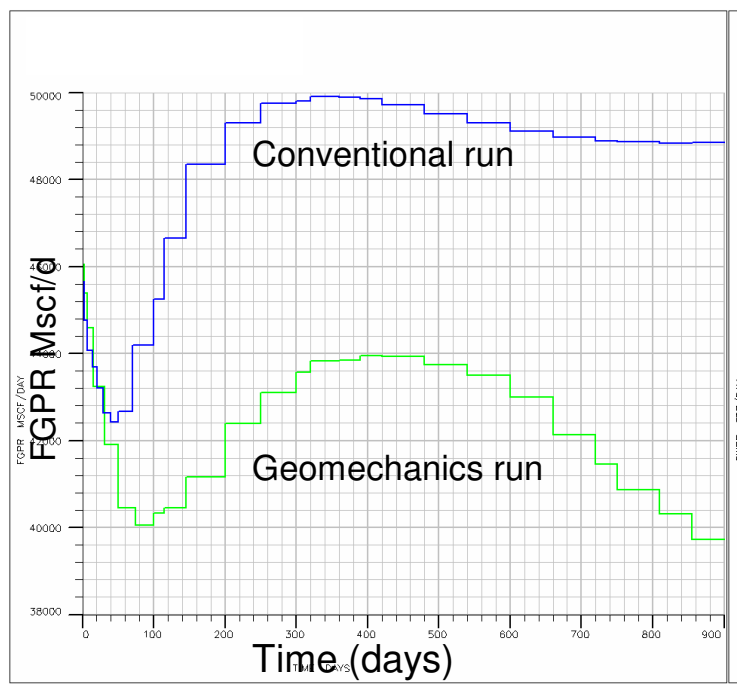

Field Oil Production Rate

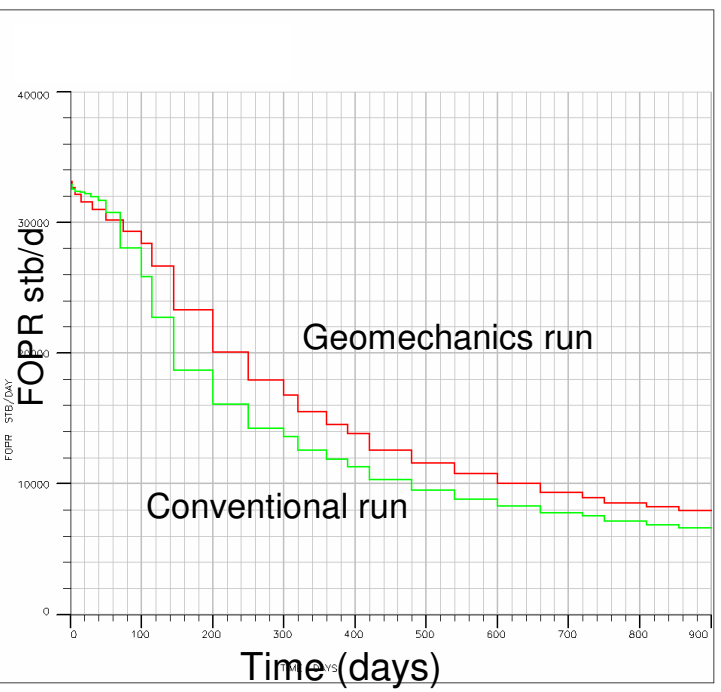

Figure 42 - Comparison of Pressure and Oil production for Runs With Geomechanics Option and Without Geomechanics Option 
It has been discussed earlier about the pressure support provided by rock compaction due to pore volume reduction. With 25 producers and 1 injection well, the SPE $9^{\text {th }}$ comparative case experiences pressure depletion throughout the reservoir during fluid production. Rock compaction acts as a reservoir drive mechanism for simulation run with geomechanics option whereas conventional run has natural depletion drive. Reservoir geomechanics cause pore volume reduction due to pore pressure depletion and this change in pore volume supports fluid pressure from further reduction. The drop in average reservoir pressure for geomechanics run is less compared to drop in average reservoir pressure for conventional run as evident from the field pressure plot. The pressure maintenance provided by rock compaction improves oil production and final oil recovery for geomechanics run.

The different path followed by fluid pressure during the course of production will influence behavior of solution gas in oil and PVT properties of oil that are directly related to the fluid pressure. For both simulation runs, the impact of variation in solution gas in oil and fluid pressure on reservoir dynamics can be understood in more comprehensive manner by visualizing flow for individual phases through streamline tracing. 


\section{$1^{\text {st }}$ Report Step}

Geomechanics Run

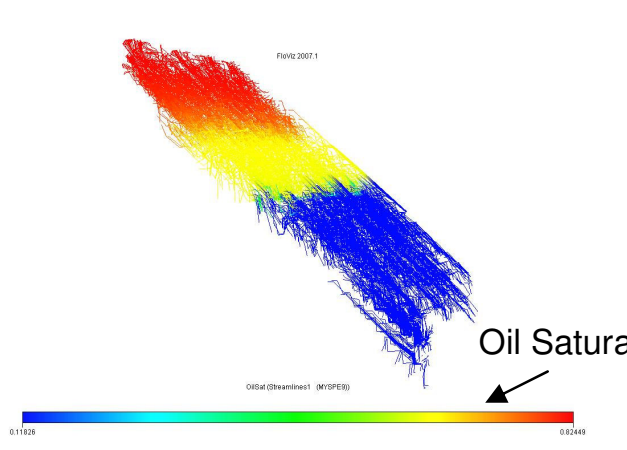

\section{Conventional Run}

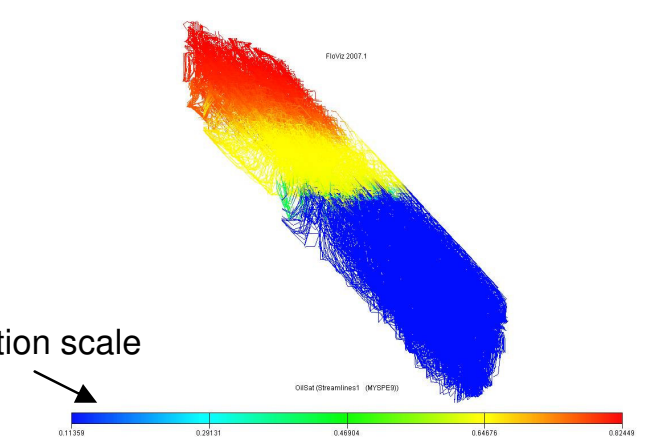

Total Velocity Tracing - 3D View

\section{Last Report Step}

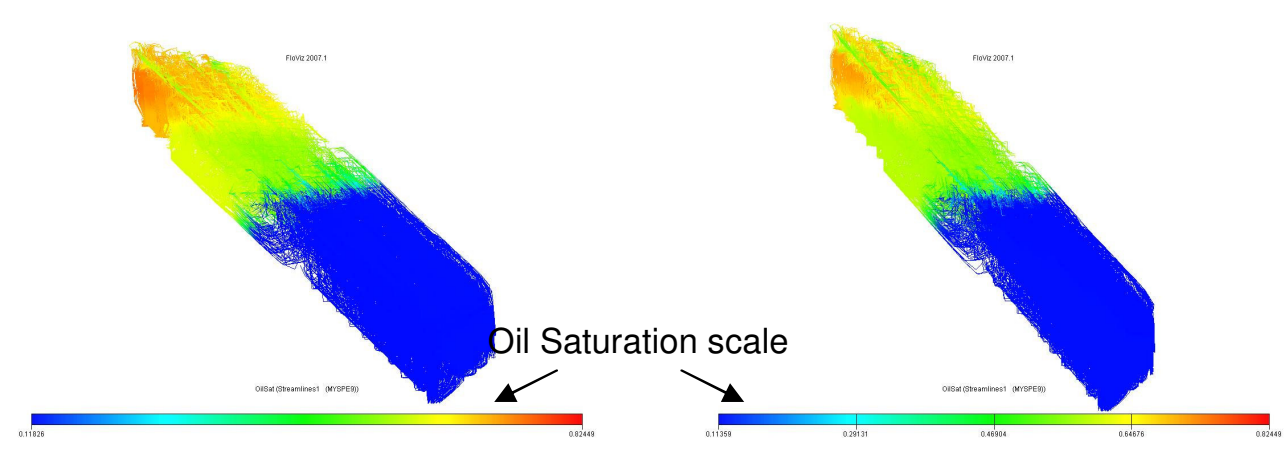

Total Velocity Tracing - 3D View

Figure 43 - Side View of the Streamline Tracing

The side view shows the total velocity tracing in dipping reservoir. The blue region shows the immovable oil saturation below the water-oil contact. In the upper part of the reservoir, the oil saturation decreases from $1^{\text {st }}$ report step to last report step due to fluid production. 


\section{$1^{\text {st }}$ Report Step}

Geomechanics Run

Conventional Run
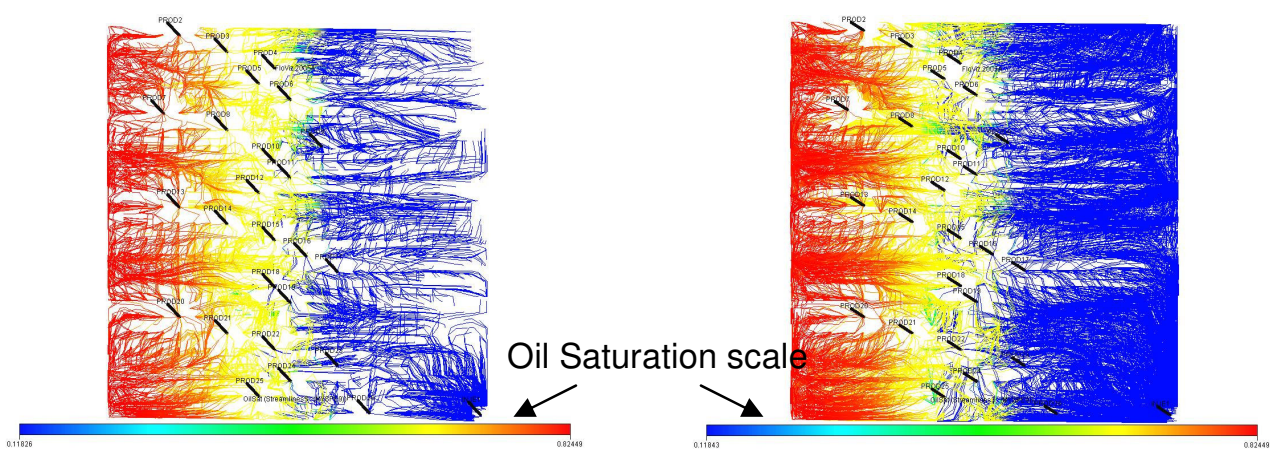

Total Velocity Tracing - Top View
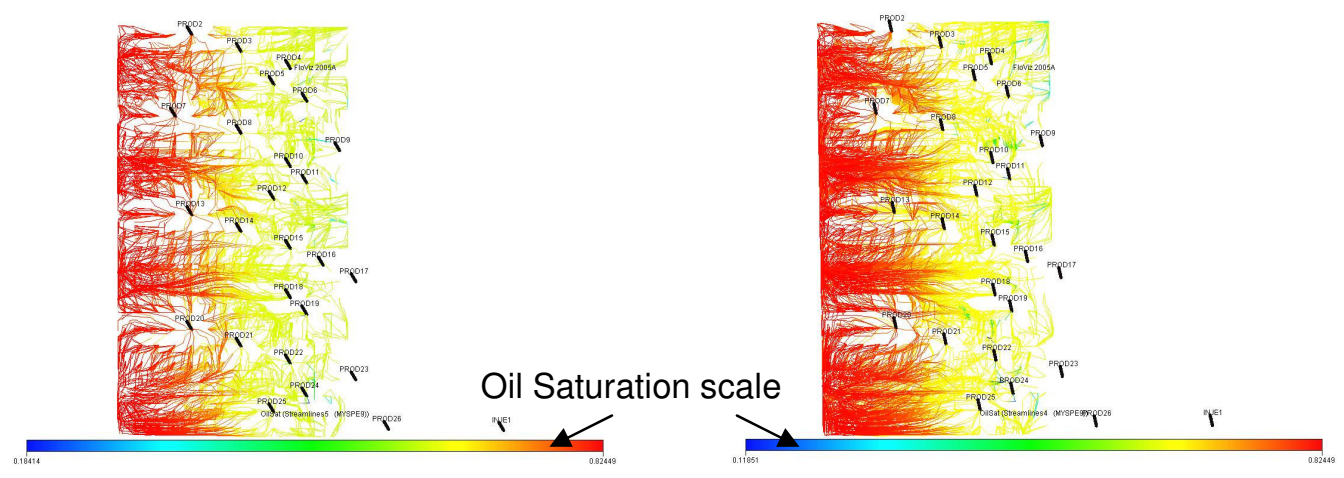

Oil Phase Tracing - Top View
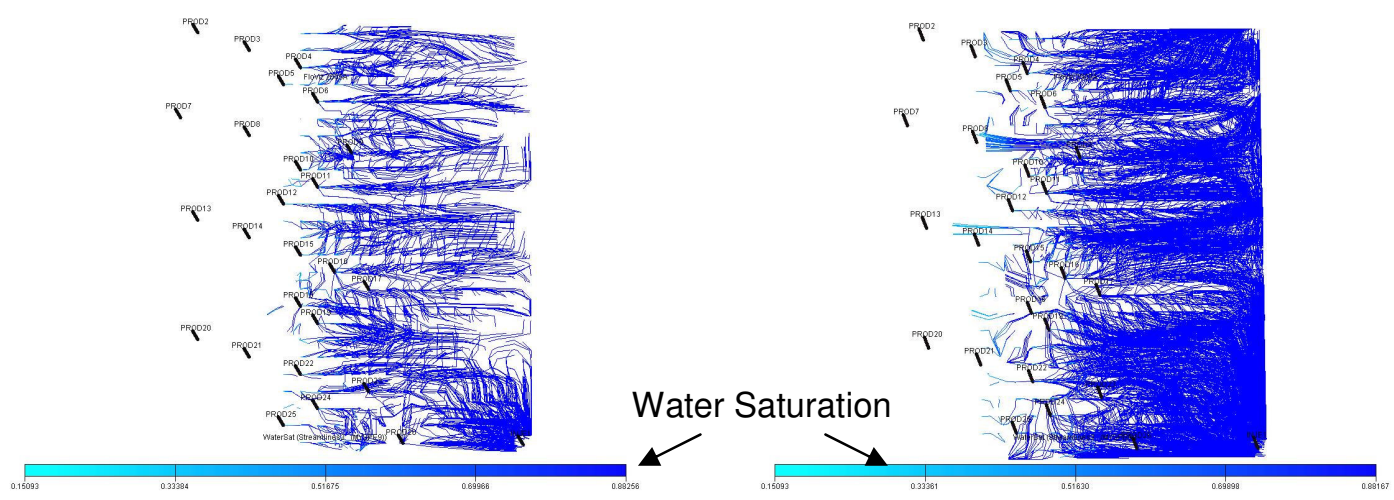

Water Phase Tracing - Top View

Figure 44 - Total Velocity Tracing and Individual Phase Tracing for $1^{\text {st }}$ Report Step 
Different plots in Figure 41 make a distinction between different parts of the reservoir based on type of phase flowing through it. The total velocity tracing gives a general idea about the flow taking place in the reservoir but individual phase tracing completely specifies in which region a particular phase is dominant in flow. For the $1^{\text {st }}$ report step, gas saturation is zero so there is no gas phase present in the reservoir so, we have not shown gas phase streamlines. An important thing to notice here is the density of streamlines. All the producers have same rate constraint and the streamline density for geomechanics run is less than streamline density for conventional run. This shows that for early report steps, flow is higher for conventional simulation and this fact is supported by the plots for field oil production rate and field water production rate. But for the field gas production rate, conventional simulation has higher rate as it has more pressure drop in the reservoir causing more gas to evolve from oil and hence more gas flow.

\section{Last Report Step}

Geomechanics Run

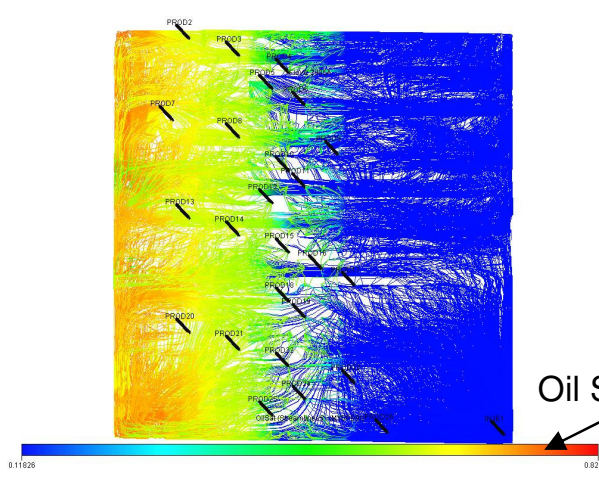

Conventional Run

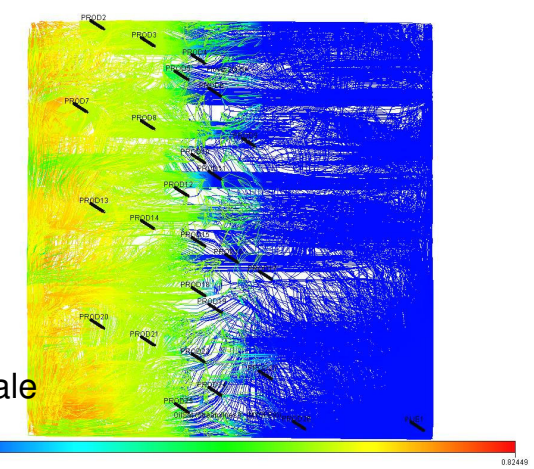

Total Velocity Tracing - Top View

Figure 45 - Total Velocity Tracing for Last Report Step 


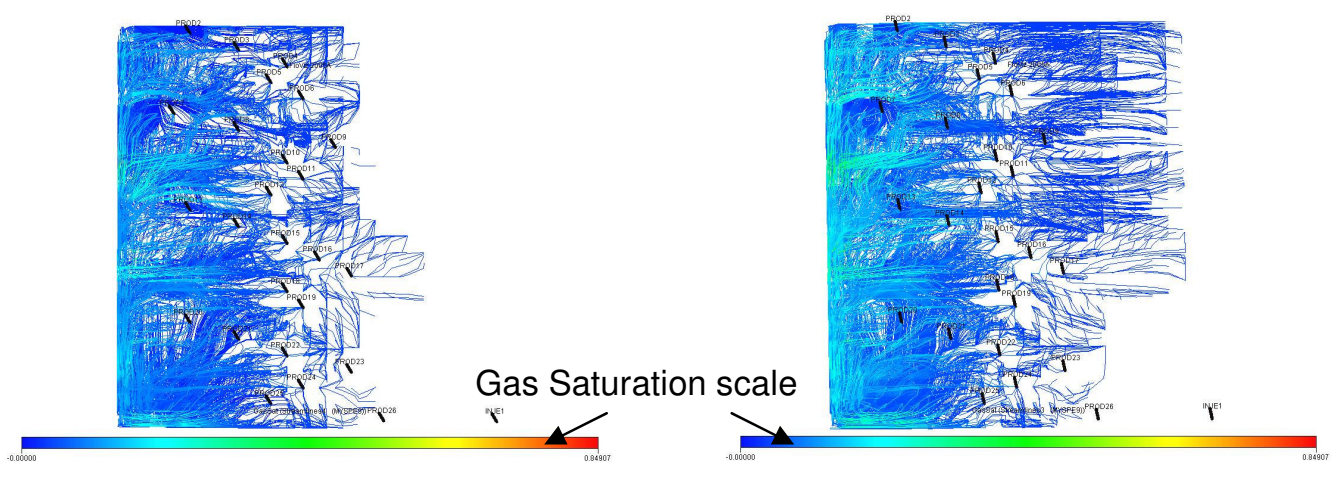

\section{Gas Phase Tracing - Top View}

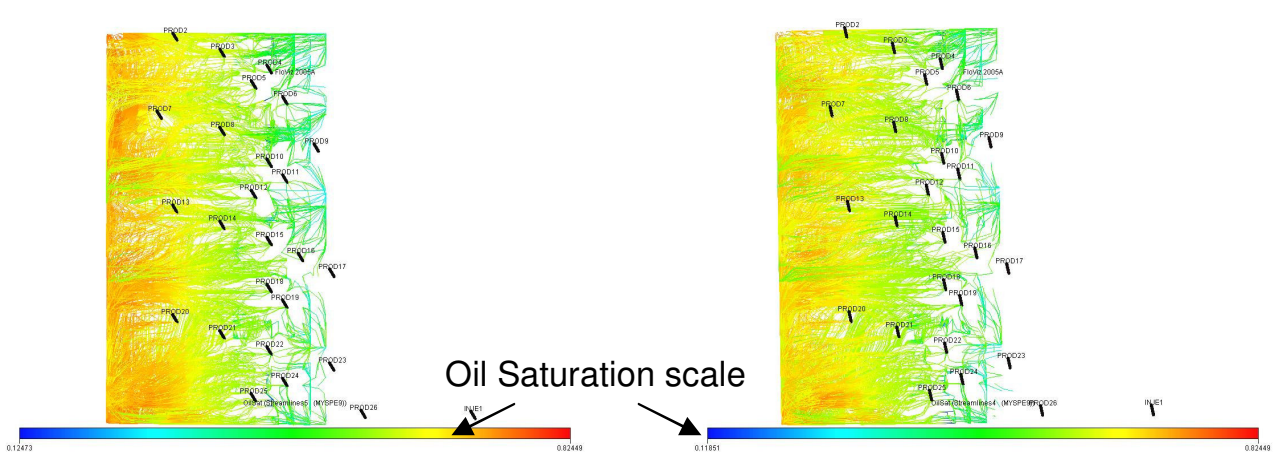

Oil Phase Tracing - Top View

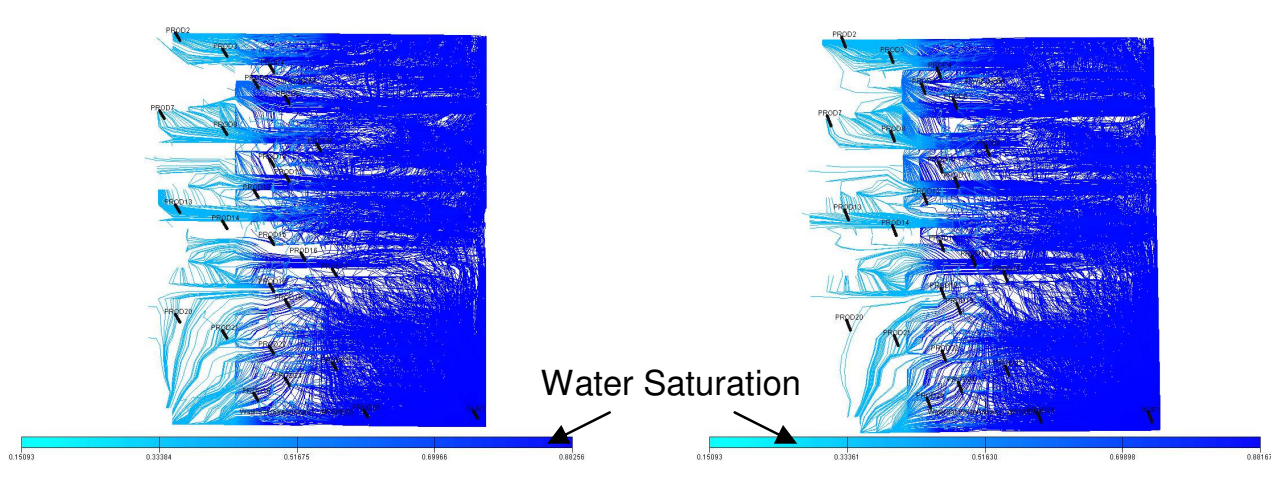

Water Phase Tracing - Top View

Figure 46 -Individual Phase Tracing for Last Report Step

The tracing of total velocity, oil phase flow for the last report step shows decrease in oil saturation due to fluid production. On the other hand, gas phase tracing provides 
view of more gas flow in conventional run. This is due to higher pressure drop in the reservoir for conventional simulation leading to release of more gas from oil phase compared to geomechanics run. The water phase tracing shows the presence of water flow in the region where previously there was no water. Based on this, it can be inferred that there is a natural water encroachment from the downdip aquifer.

\subsubsection{Comparison of Production for Producer 19}

Well Gas Rate

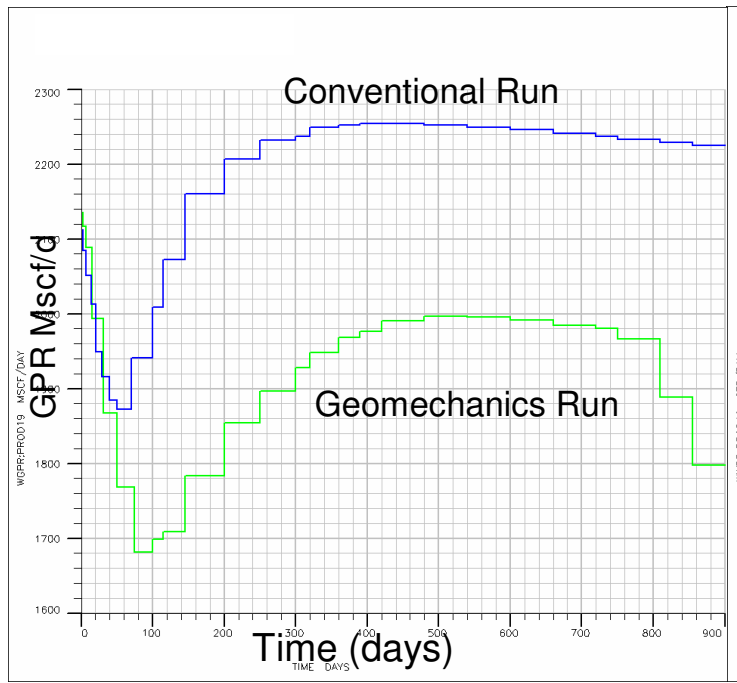

Well Water Rate

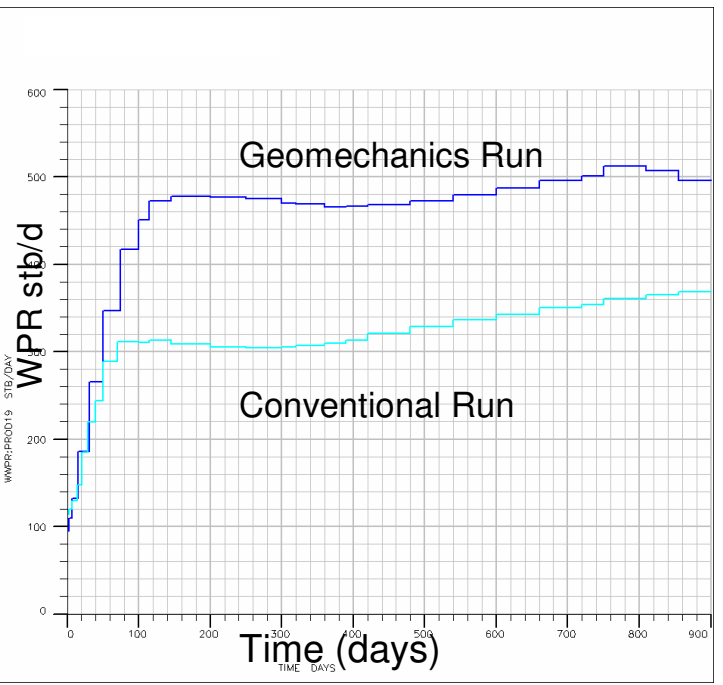

Figure 47 - Gas Production Rate and Water Production Rate for Producer 19

The plot for gas production shows that for geomechanics run gas flow rate is lower whereas water production rate is higher compared to conventional run. Visualization of flow near the well will provide the reasons for the differences in flow rates for the two simulation runs. 
Geomechanics Run

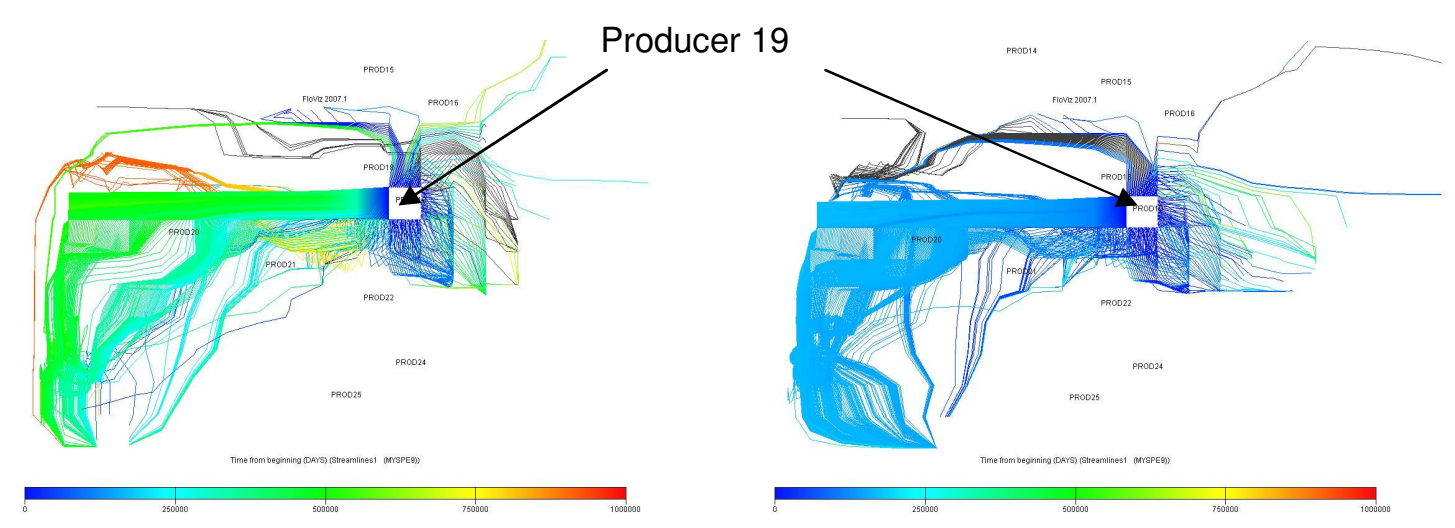

Figure 48 - Time of Flight for Gas Phase Tracing at Last Report Step - Top View

Time of flight picture for gas phase tracing shows gas flow takes higher time in geomechanics run to reach the well compared to gas flow in conventional run. The reason for gas flow being faster in conventional run is higher pressure drop in the reservoir due to lack of pressure support.

Geomechanics Run

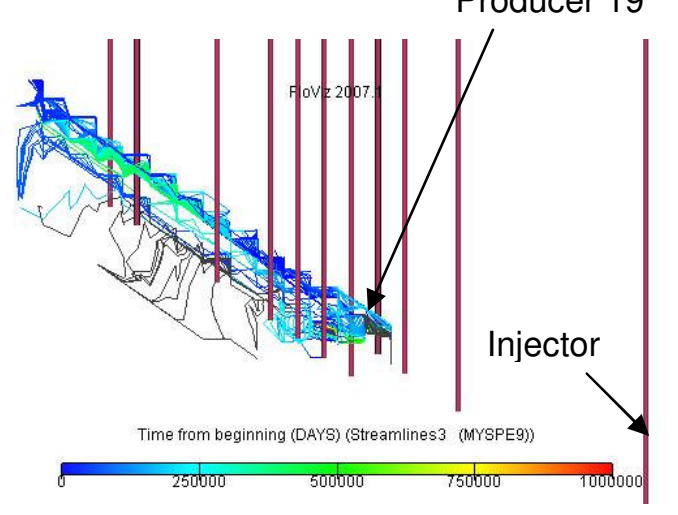

Conventional Run

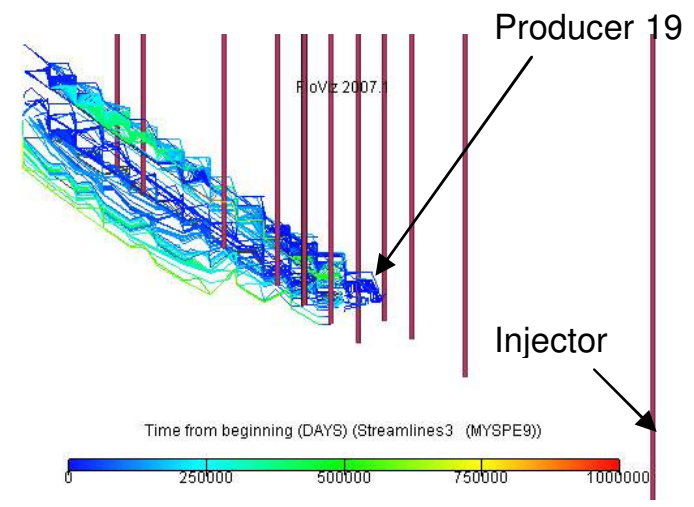

Figure 49 - Time of Flight for Gas Phase Tracing at Last Report Step - Side View 
Side view of the gas phase tracing reveals more information about differences in flow pattern between two simulators. Streamlines for the conventional run shows that the large number of layers is contributing to gas flow towards producer 19 whereas in geomechanics run few layers have gas flow.

\section{Geomechanics Run}

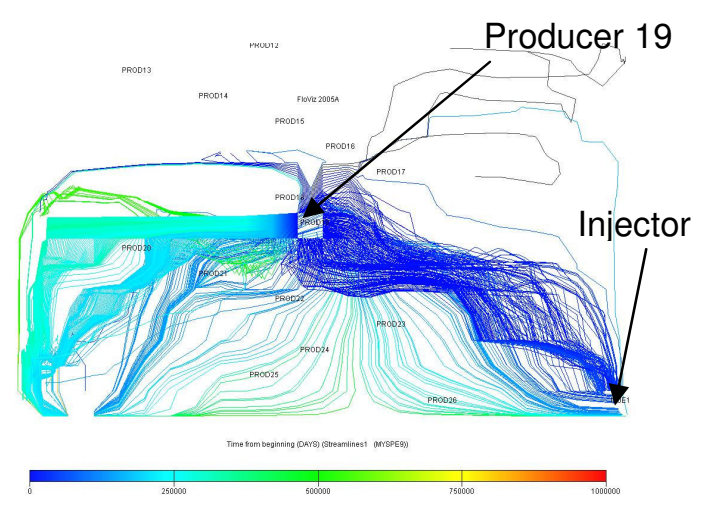

\section{Conventional Run}

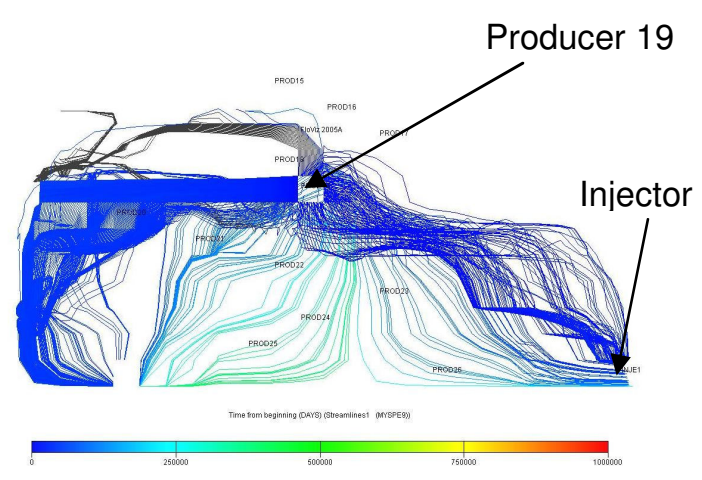

Figure 50 - Time of Flight for Total Velocity Tracing at Last Report Step - Top View

The picture for total velocity tracing for both simulation runs does not show any major difference in flow pattern. Time of flight in case of conventional run is small for water flow but plot for water rate shows that water production for producer 19 is higher for geomechanics case. So from the above picture, the higher water production rate for geomechanics run compared to conventional run cannot be explained. 
Geomechanics Run

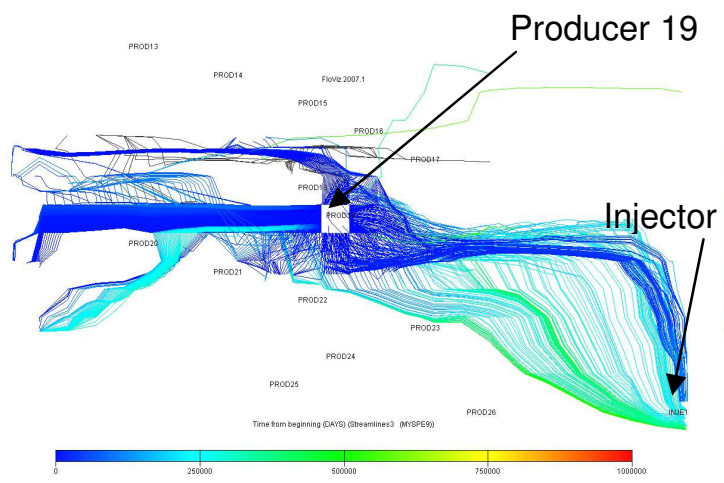

Conventional Run

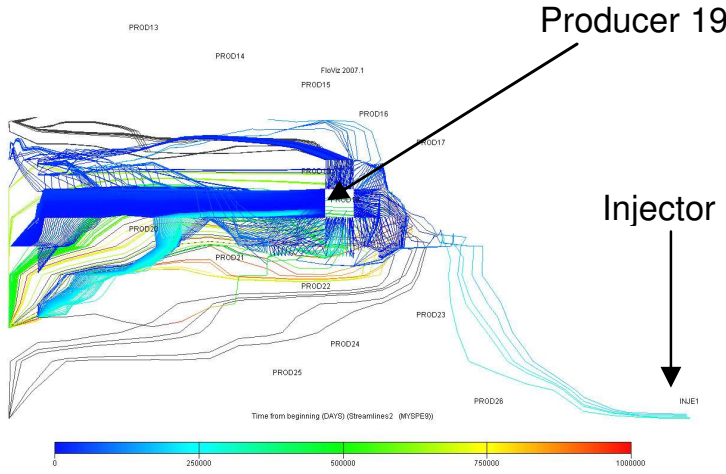

Figure 51 - Time of Flight for Total Velocity Tracing at $3^{\text {rd }}$ Report Step - Top View

Comparison of streamlines for both simulation runs explains the reason behind higher water production for geomechanics run. Large number of streamlines from injector to producer 19 suggests that more water is flowing towards the producer in geomechanics run. In conventional run, it's clear that small flow is taking place from injector to produce 19 .

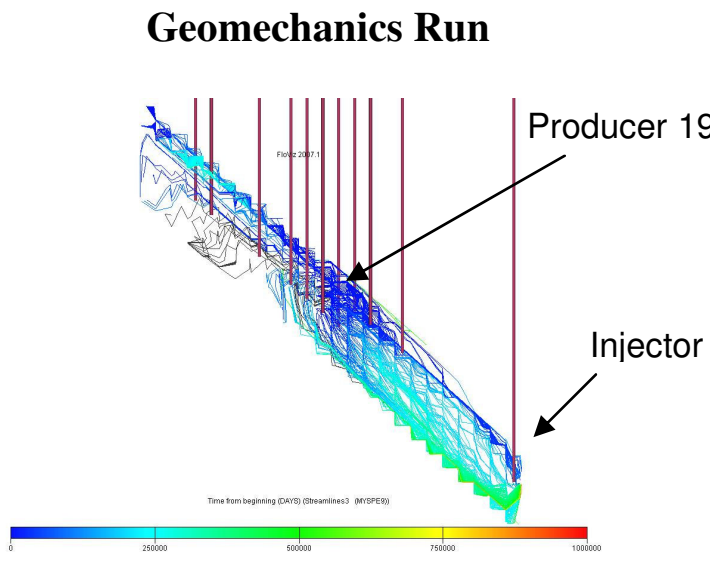

Conventional Run

Producer 19

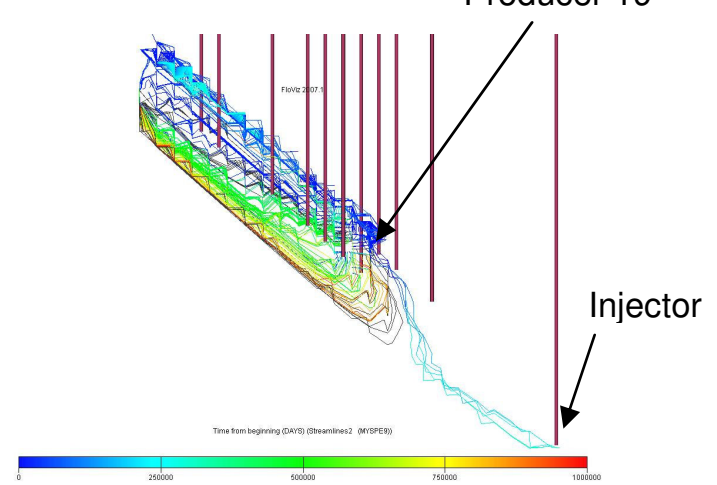

Figure 52 - Time of Flight for Total Velocity Tracing at $3^{\text {rd }}$ Report Step - Side View 
Side view of the streamline tracing shows the difference in flow pattern between both simulation runs. In geomechanics run, all the layer are contributing to water flow whereas in conventional run, water flow is taking place in lower most layers. As a result, there is high water production in producer 19 for geomechanics run.

\subsubsection{Comparison of Production for Producer 4}

Well Gas Rate

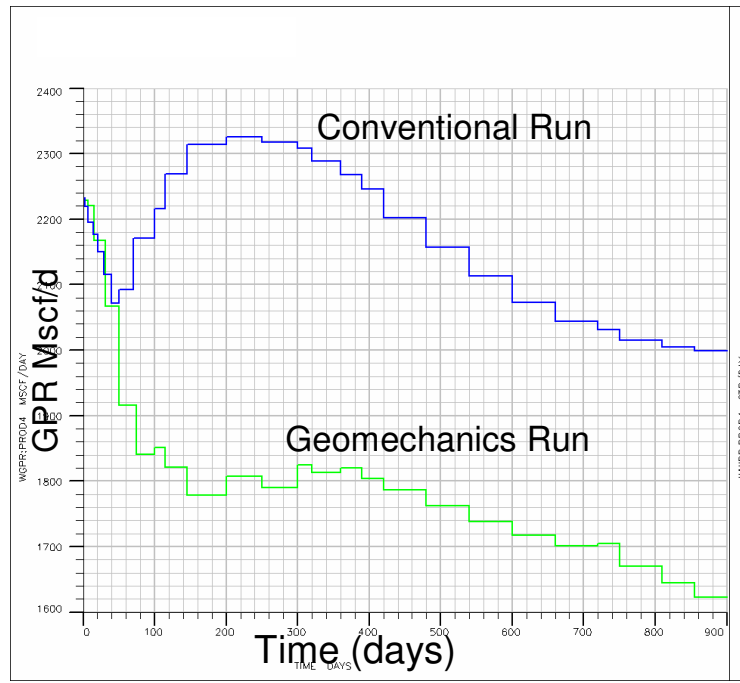

Well Water Rate

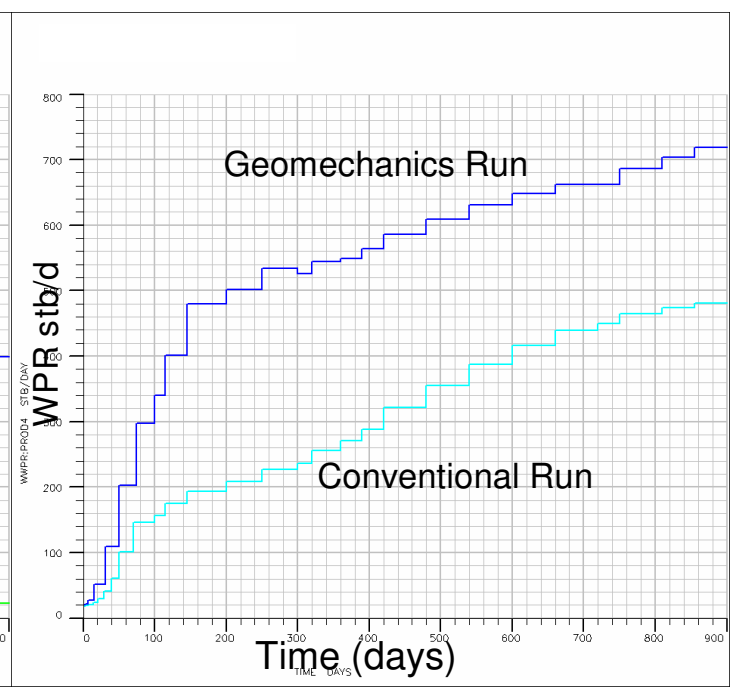

Figure 53 - Gas Production Rate and Water Production Rate for Producer 4

The trend of gas production is same as discussed previously for produce 19 , the gas rate being higher for conventional run. Plot for water production is also similar compared to producer 19 but the streamline profile for producer 4 shows that the flow pattern near the well is different than the flow pattern for producer 19. 


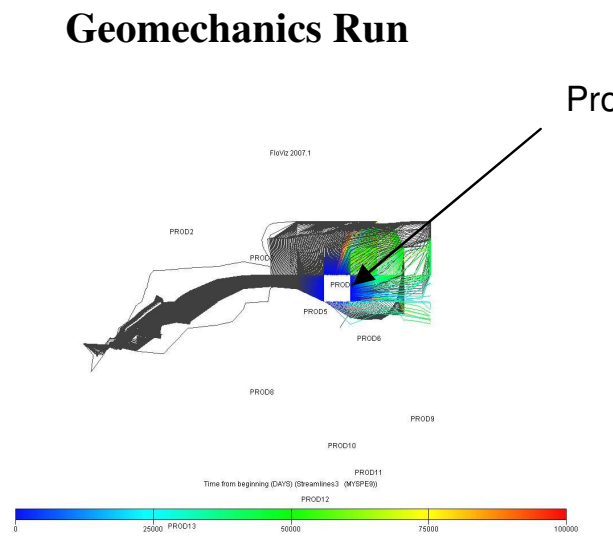

\section{Conventional Run}

Producer 4

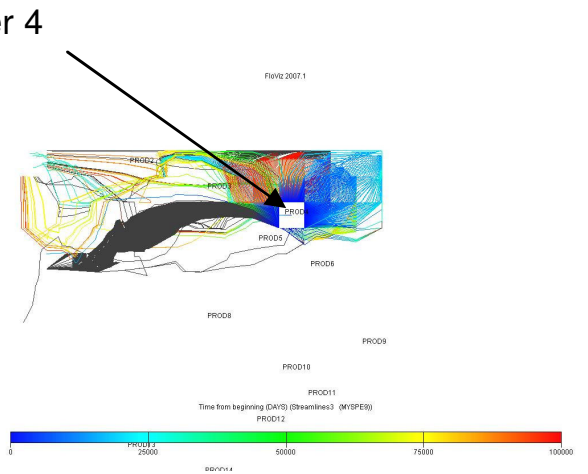

\section{Figure 54 - Time of Flight for Gas Phase Tracing at $5^{\text {th }}$ Report Step - Top View}

At $5^{\text {th }}$ report step, time of flight for gas phase tracing in conventional run is small compared to geomechanics run and we see a higher flow of gas towards producer 4 . The trend continues for streamline profiles at last report step. Gas phase tracing for conventional run shows that gas flow is taking place in larger area compared to geomechanics run and the time of flight is smaller for conventional run. These pictures support the nature of gas rate profiles for the two simulations.

Geomechanics Run

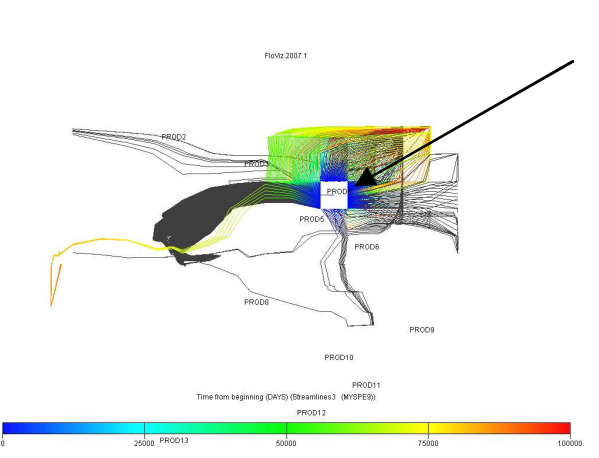

Conventional Run

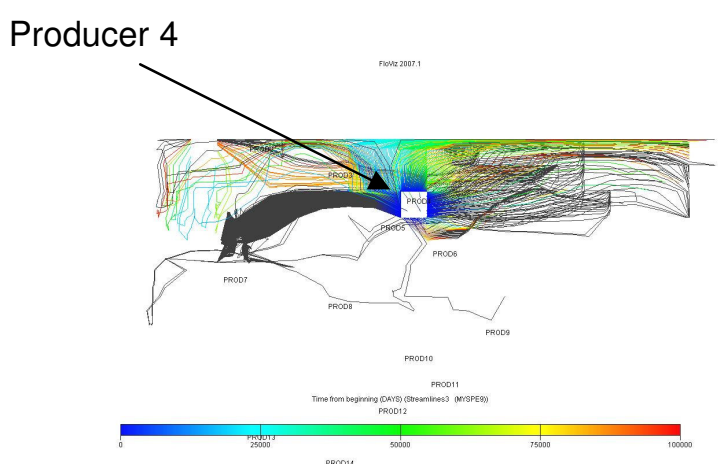

Figure 55 - Time of Flight for Gas Phase Tracing at Last Report Step - Top View 

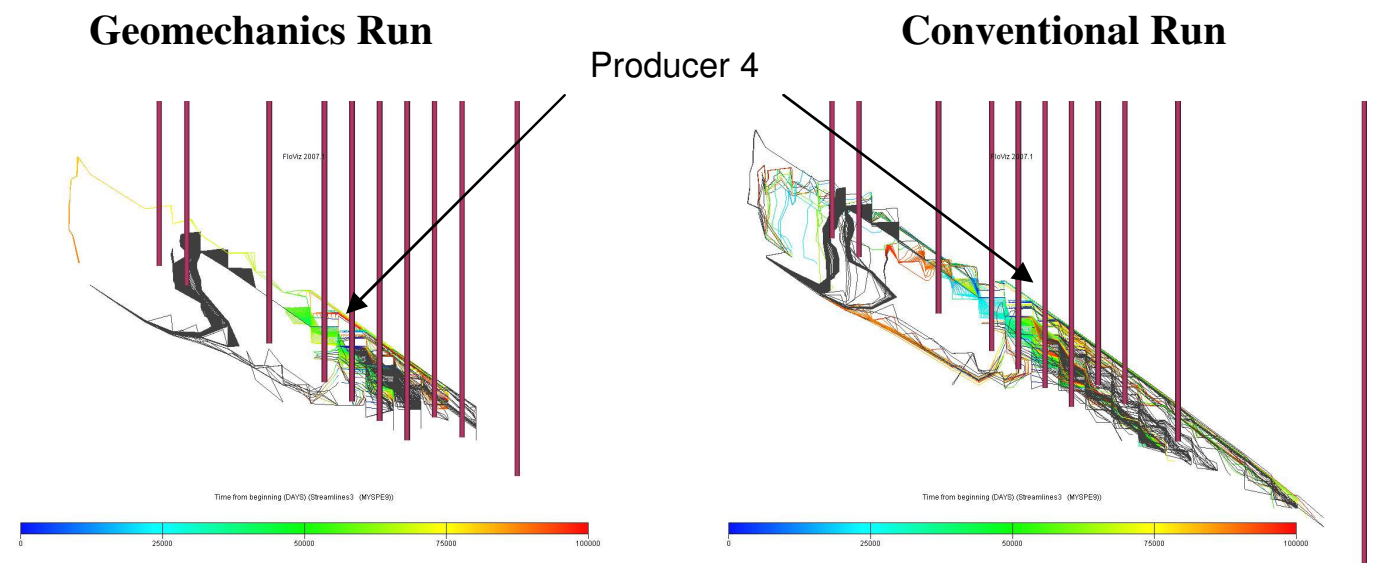

Figure 56 - Time of Flight for Gas Phase Tracing at Last Report Step - Side View

Comparison of side view of the gas phase tracing for both simulation runs shows the difference in layer wise flow pattern. In geomechanics run, gas flow is not taking place in all the layers whereas in case of conventional run there is gas flow in most of the layers. So, geomechanics is influencing gas flow in layers through changes in pore volume and permeability. 
The streamline tracing represents underlying flux distribution in the reservoir and the flux depends on the pressure field. So the major factor driving the streamline profiles in the pictures above is the pressure and its variation in the reservoir.
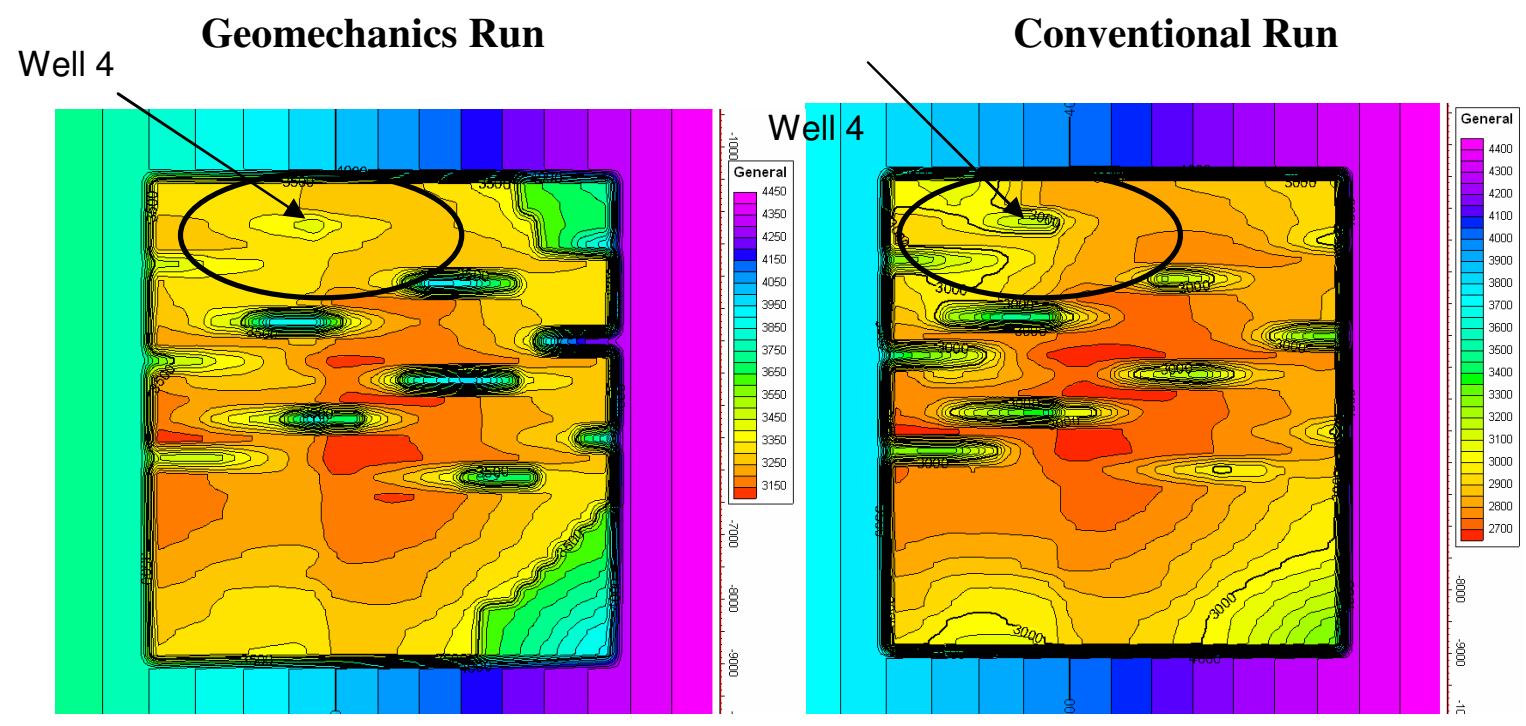

Figure 57 - Gas Phase Pressure at Last Report Step

The gas phase pressure contours for the last layer and for the last report step shows the variation in pressure around the producer. The presence of less number of contours in the circled area around well 4 suggests that the geomechanics run has less pressure variation compared to conventional run which has high pressure variation. Flow potential is one of the parameters on which the value of flux depends. Flow potential is calculated from pressure variation and the simulation with less pressure variation will result in low flow potential, ultimately leading to lower flux. 
Geomechanics Run Conventional Run

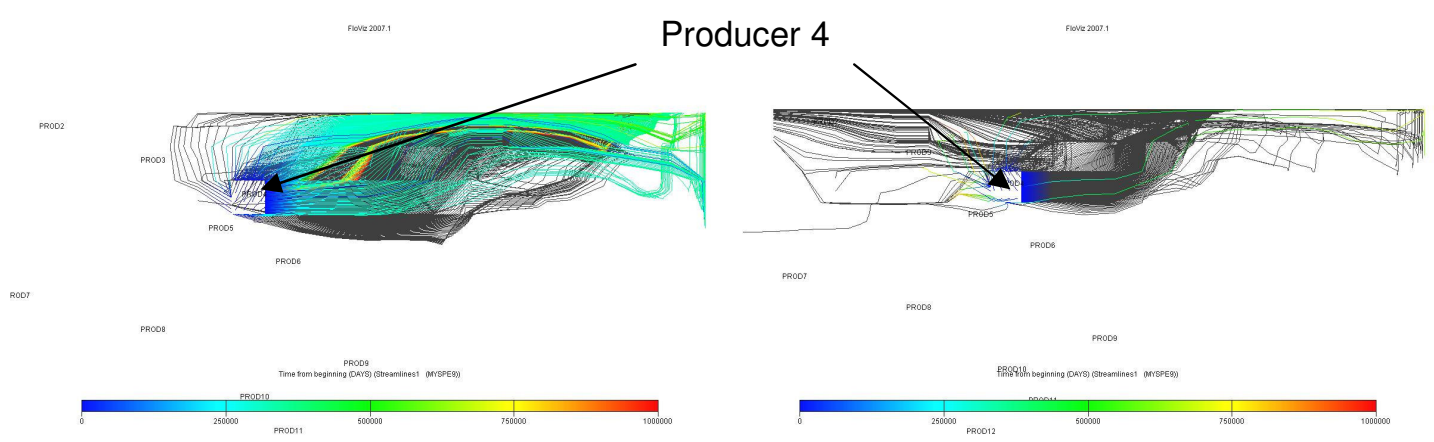

Figure 58 - Time of Flight for Water Phase Tracing at $4^{\text {th }}$ Report Step - Top View

The water production rate for producer 4 is higher for geomechanics run as seen in the plots for well rates presented previously. The streamline tracing for water phase also shows that for geomechanics run, the time of flight for water flow is less. In terms of flow direction, there is no major difference between the two simulations.
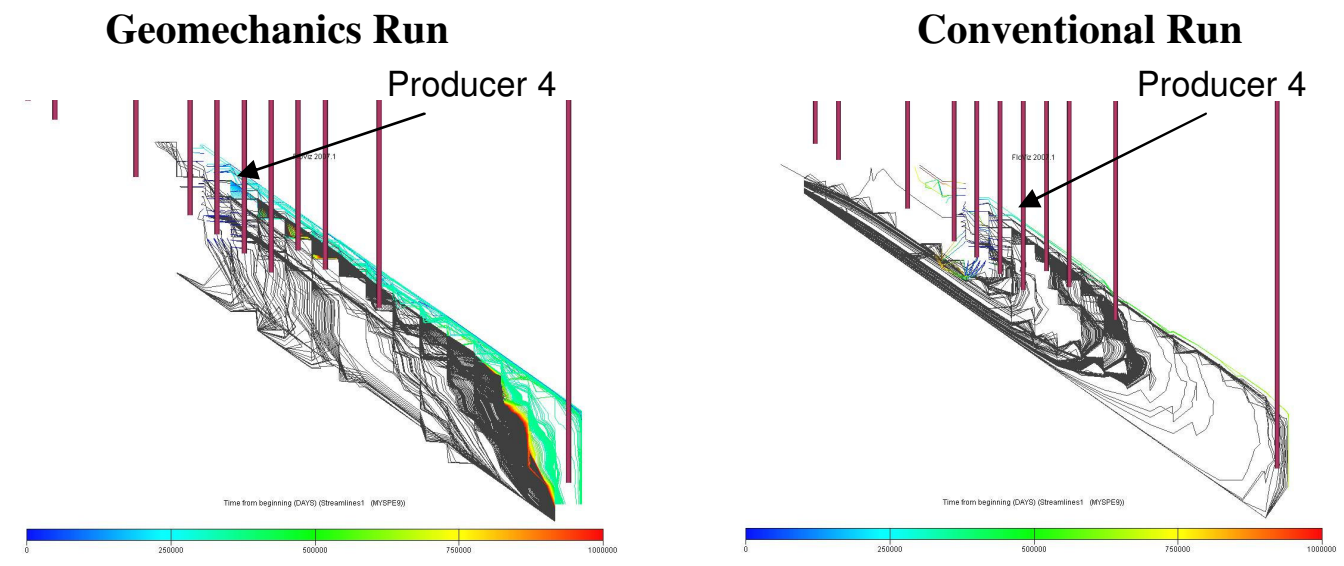

Figure 59 - Time of Flight for Water Phase Tracing at $4^{\text {th }}$ Report Step - Side View 
Side view of streamline tracing for water phase shows two prominent features. First, all the layers in geomechanics run are contribution to water flow whereas in conventional run, flow is taking place in few layers. Second, time of flight for water phase tracing is smaller for upper layers in geomechanics run. These factors also support the higher production rate of water in producer 4 . The reason for these differences in layer wise flow pattern is the variation in water phase pressure between the layers for both simulation runs.

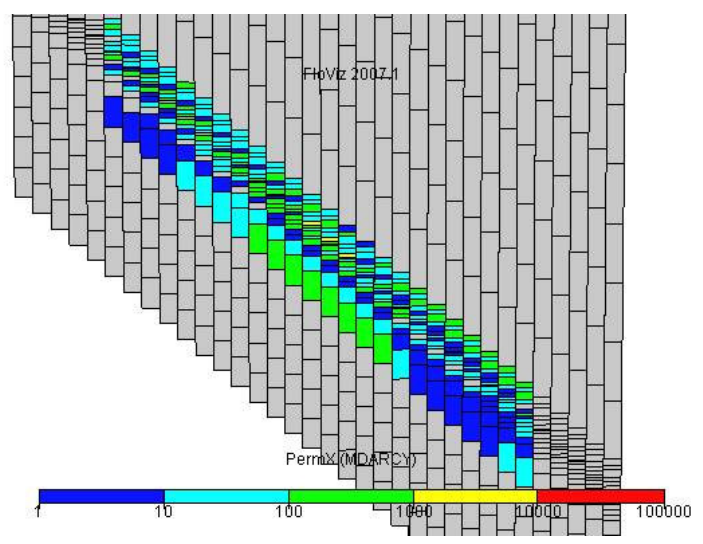

Figure 60 - Permeability Distribution for All the Layers - Side View

In geomechanics run, the average pressure in layers towards the upper part of the reservoir is $3000 \mathrm{psi}$ and average pressure in bottom layers is around 3500 psi. Comparing these pressures with conventional run results, the average pressure in upper layers is around 2700 psi and average pressure in bottom layers is 2900 psi. So, pressure variation in vertical direction for geomechanics run is higher than pressure variation for conventional run. This pressure variation which causes flow potential to be higher, provides the reason behind the smaller time of flight for flow of water from bottom of the reservoir towards top of the reservoir in geomechanics run. 
Geomechanics Run

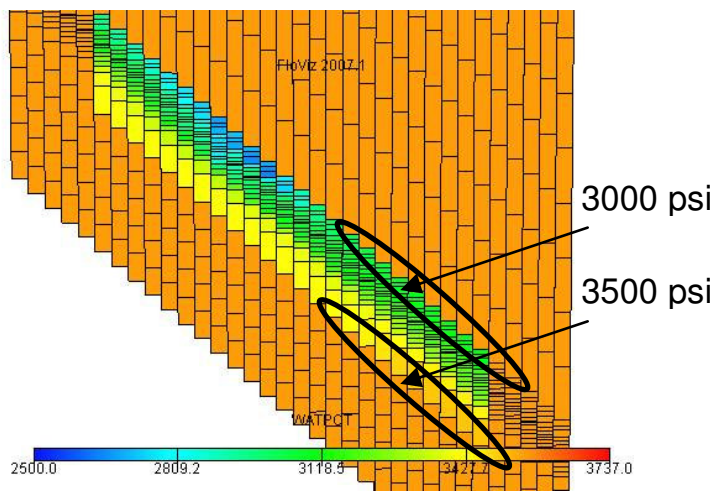

Conventional Run

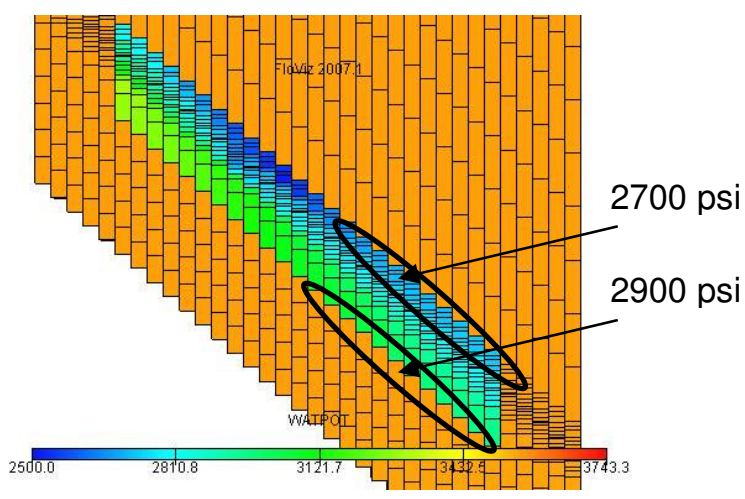

Figure 61 - Water Phase Pressure at $4^{\text {th }}$ Report Step for All the Layers - Side View

The water phase pressure contours for the first layer at $4^{\text {th }}$ report step shows the variation in pressure around the producer. The presence of less number of contours in the circled area around well 4 suggests that the geomechanics run has higher pressure variation compared to conventional run in the top layer. This also supports the reason behind smaller time of flight for water phase tracing and higher flow rate for producer 4 in geomechanics run.

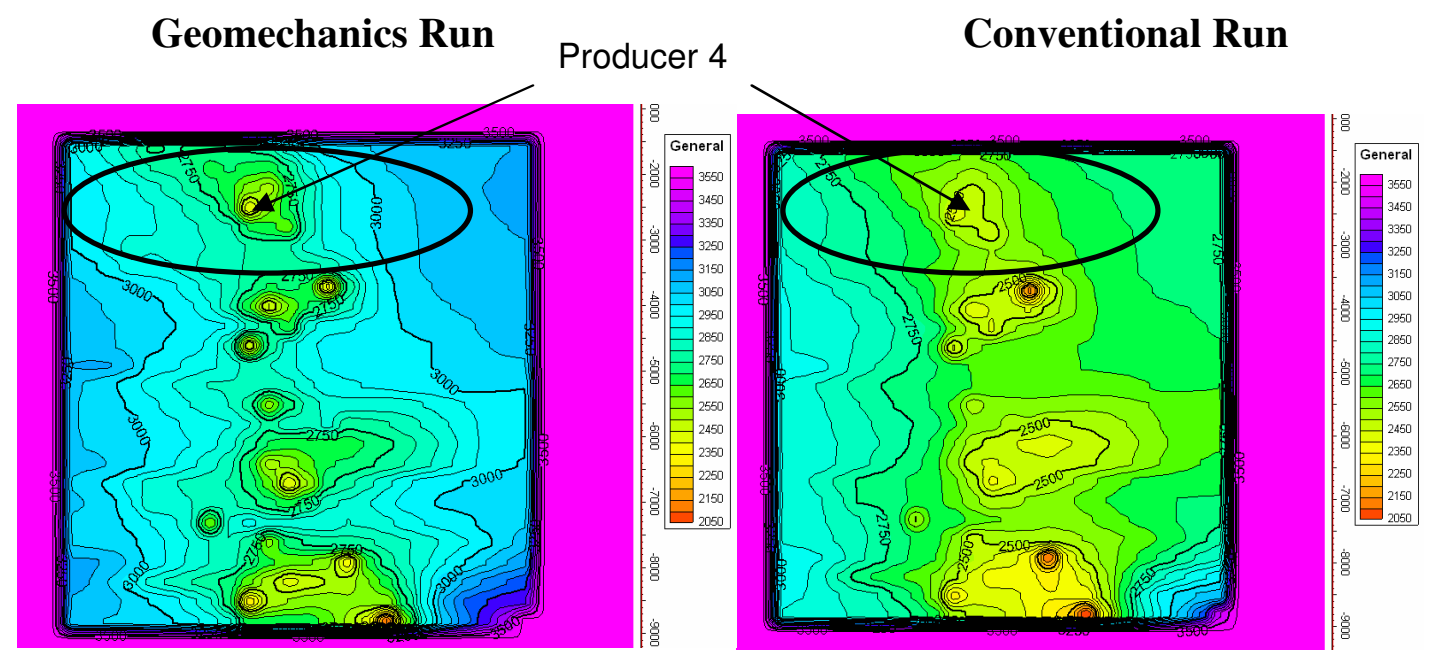

Figure 62 - Water Phase Pressure at $4^{\text {th }}$ Report Step for First Layer 


\subsubsection{Water Injection Rate Comparison}

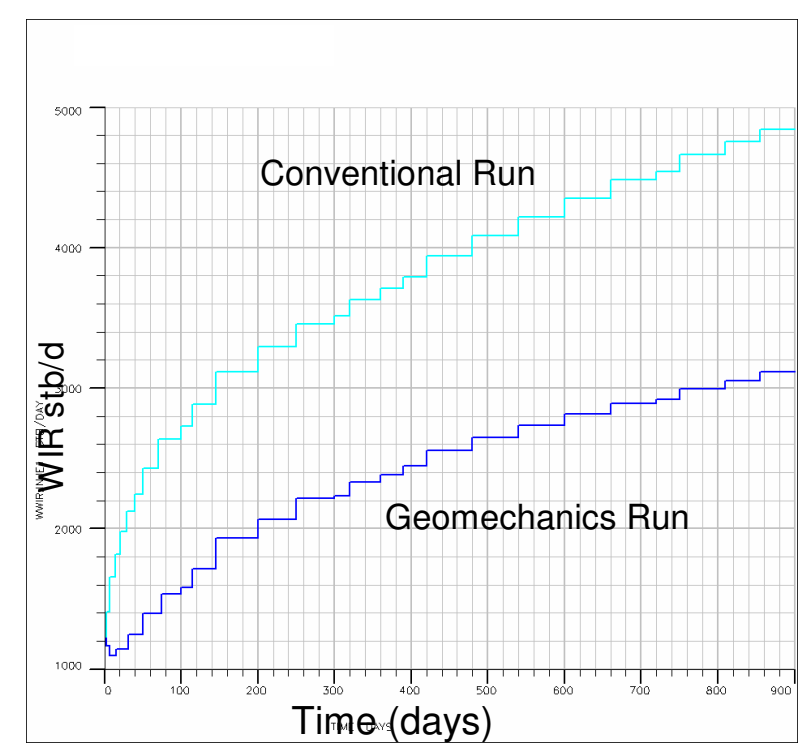

Figure 63 - Water Injection Rate for Injector

Figure 56 compares water injection rates for injector in both simulation runs. Water production for the field and water production from the producers is higher for geomechanics run as shown in the plots presented previously but here, the water injection rate for conventional run is higher compared to geomechanics run. The reason for this is the pressure drop in the reservoir. In both simulations, the injection rate is controlled by limiting the maximum bottom hole pressure for the injection well. So, higher drop in reservoir pressure for conventional run leads to bottom hole pressure for the injector to be of lower value and hence allowing for more injection rate. 


\section{Geomechanics Run}

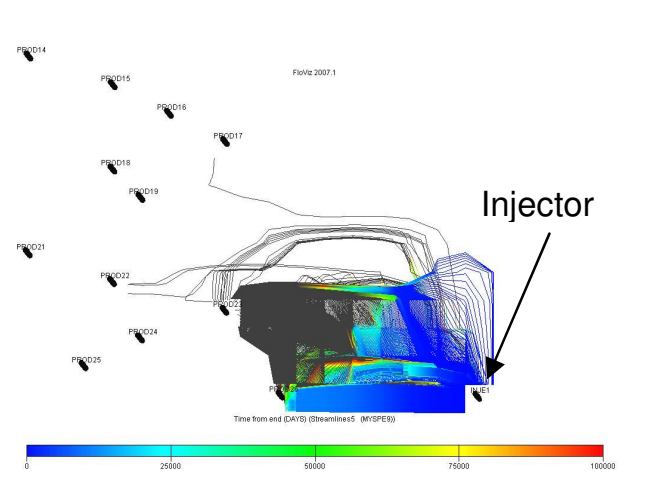

\section{Conventional Run}

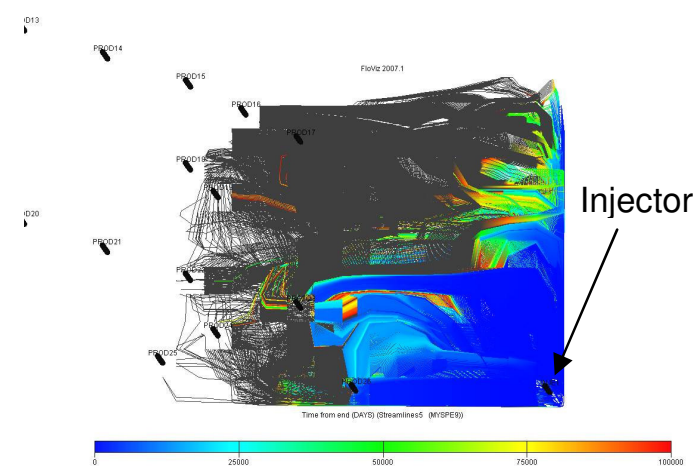

Figure 64 - Water Phase Tracing for Injector at $1^{\text {st }}$ Report Step

The water phase streamlines for $1^{\text {st }}$ report step shows the same result as seen in the plot for water injection rate in figure 56. Streamlines in conventional run covers much larger area compared to geomechanics run which suggests faster movement of waterflood due to higher injection rate.

Geomechanics Run

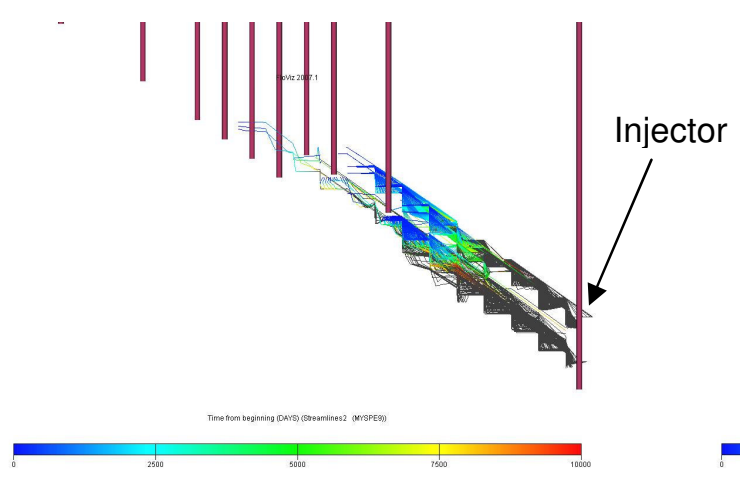

Conventional Run

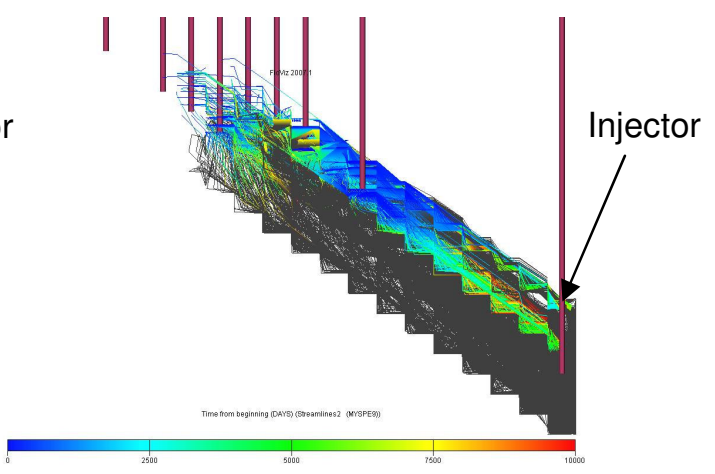

Figure 65 - Time of Flight for Water Phase Tracing at $1^{\text {st }}$ Report Step - Side View

Above picture for the water phase streamlines at $1^{\text {st }}$ report step shows that in geomechanics run there is no flow of water in certain layers but for conventional run, the water is flowing in all the layers. This difference in streamlines is due to the flux 
distribution pattern. The main driving factor in streamline tracing is pressure and its variation in the reservoir as it influences calculation of flux. So comparison of pressure distribution for the two simulations will provide information regarding the differences in water flow for both simulations.

Geomechanics Run

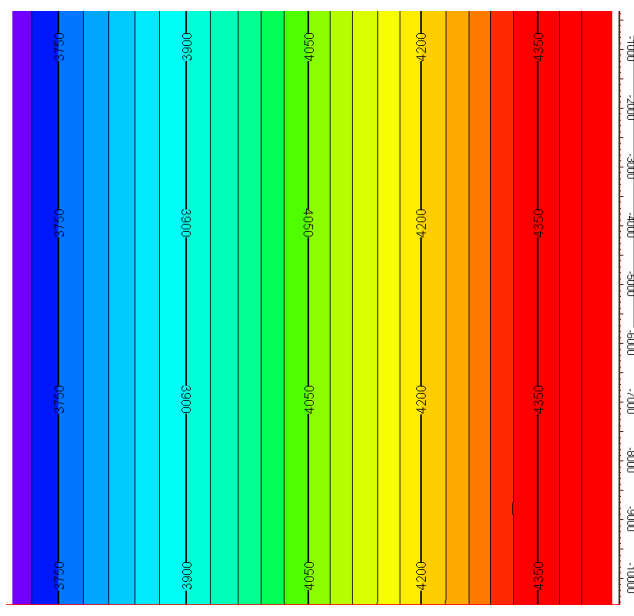

Figure 66 - Reservoir Pressure at $1^{\text {st }}$ Report Step - Top View
Conventional Run

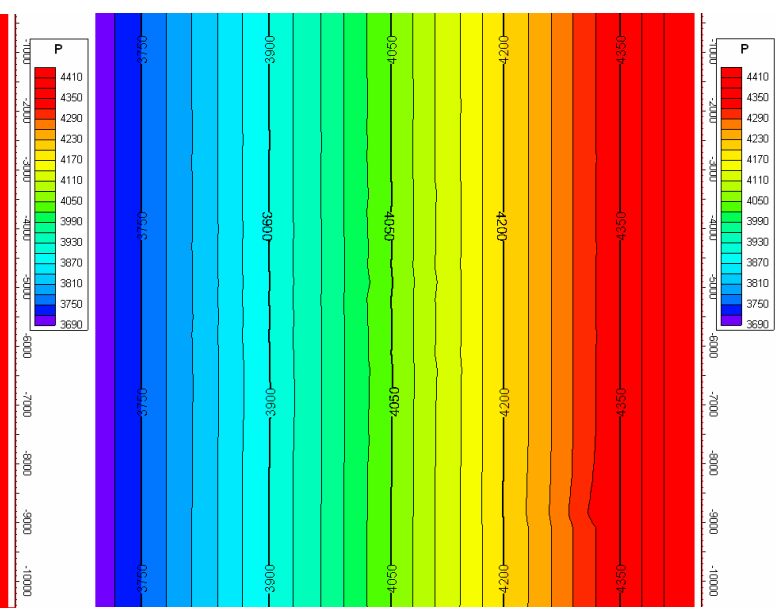

The contours for reservoir pressure shows that both simulations have exactly the same pressure distribution so one needs to look at the individual phase pressure contours to determine the differences obtained in water flow at $1^{\text {st }}$ report step.

The pressure contours shown below for water phase reveals the reason behind lack of water flow in the some of the layers for the geomechanics run. 
Geomechanics Run

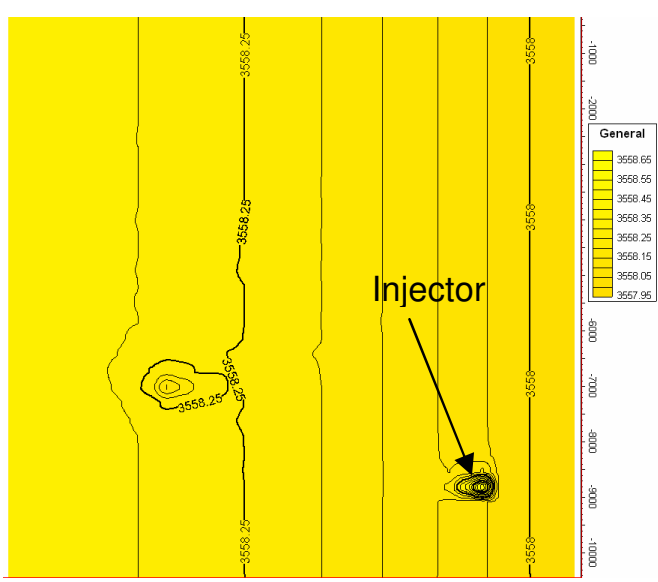

\section{Conventional Run}

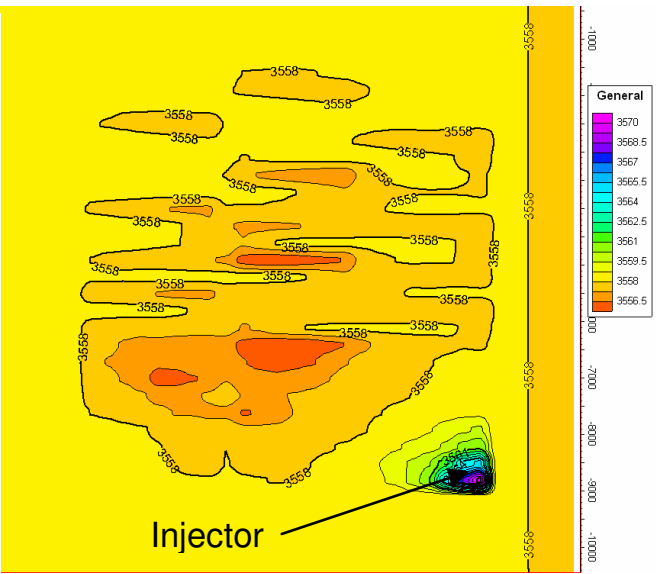

Injector

Figure 67 - Water Phase Pressure at $1^{\text {st }}$ Report Step for Last Layer

Geomechanics Run
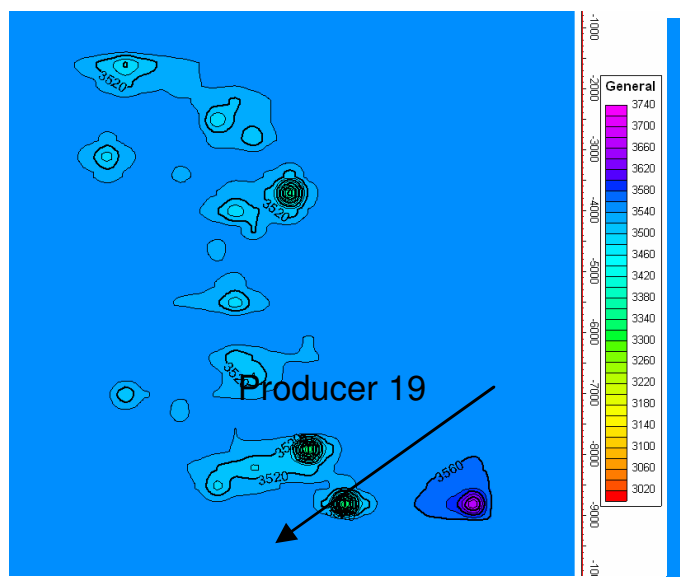

Conventional Run

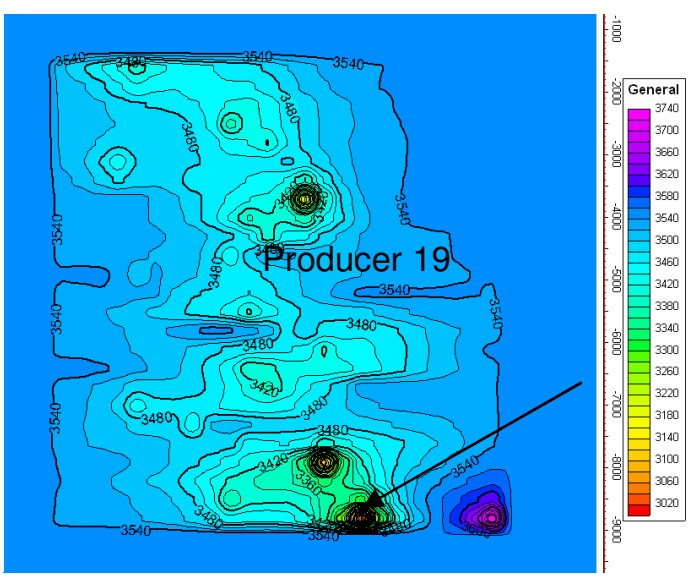

Figure 68 - Water Phase Pressure at $1^{\text {st }}$ Report Step for First Layer

It is clear from the picture that water phase pressure for geomechanics run has very small variation and the variation is only near the wells. This causes the flow potential to be very negligible in geomechanics run and hence no water flow in layers which such pressure distribution. 


\section{CHAPTER V}

\section{CONCLUSIONS}

1. Utilization of streamlines to visualize and compare flow patterns for coupled geomechanical simulation and conventional reservoir simulation has been successfully demonstrated for a synthetic case and SPE $9^{\text {th }}$ comparative study.

2. In-house streamline tracing tool has been successfully coupled with industry standard coupled geomechanical simulator. Phase and component streamlines in addition to total velocity streamlines acts as an efficient reservoir management tool to understand reservoir dynamics and flow patterns.

3. The fluid flow governing equation is same for both deformable and nondeformable medium at the fundamental level. The only difference is that the former requires a total derivative interpretation of the rate of change of fluid density and pore volume whereas later only requires a partial derivative.

4. The conventional reservoir simulators do not consider coupling of fluid flow and rock deformation, so they simplify important geomechanical aspects that can impact reservoir productivity. The only rock mechanical parameter involved in reservoir simulations is pore compressibility which is not sufficient in representing rock deformation as this requires full constitutive relations and influence of the surrounding nonpay formations.

5. Flow simulation and mechanical deformation modeling must be coupled to understand the response of reservoirs to fluid production and to guide well placement and production. 
6. Defining a fully coupled model for complex multiphase flow and large nonlinear inelastic deformation is costly and time consuming. Loose coupling between flow simulation and geomechanics provides best alternative to fully coupled approach due to advantages like simple to implement and fast computation.

7. The primary type of interaction between fluid flow and rock deformation is the stress induced permeability changes which in turn affect fluid-pressure distribution.

8. There is no option to define variation of anisotropic permeability with rock stresses. So the changes in permeability due to reservoir geomechanics are assumed to be isotropic.

9. The result of the two case studies shows that reservoir geomechanics influences fluid production mainly through pore volume change and the subsequent impact of pore volume change on pore pressure.

10. Reservoirs undergoing pressure depletion due to fluid production will experience pressure support through rock compaction whereas reservoirs with water injection will experience smaller pressure support due to increasing pore volume.

11. Flow pattern visualization through streamline tracing provides clear distinction in terms of flow direction between the geomechanics simulation and conventional simulation. The post processing tools with present industry standard simulators lack the ability to directly visualize the fluid flow direction in the reservoir.

12. Streamline tracing for different phases has been successfully implemented and it gives the idea on the impact of geomechanics on flow of a particular phase compared to conventional simulation. Individual phase tracing have been able to show dominant phase flowing in particular regions of the reservoir. 
13. Streamline time of flight provides clear comparison of the flood front movement for coupled geomechanical simulation and conventional simulation and also tells about the underlying permeability distribution. High permeability channels can be distinguished from low permeability regions through time of flight and streamline trajectories. 


\section{NOMENCLATURE}

\begin{tabular}{|c|c|}
\hline$\rho$ & fluid density \\
\hline$v$ & fluid velocity \\
\hline$c$ & fluid compressibility \\
\hline$v_{s}$ & solid phase velocity \\
\hline$\nabla$ & gradient operator \\
\hline$\nabla$. & Divergence operator \\
\hline$\phi$ & effective porosity \\
\hline$p$ & fluid pressure \\
\hline$t$ & time \\
\hline$V_{b}$ & bulk volume \\
\hline$V_{p}$ & pore volume \\
\hline$V_{s}$ & solid volume \\
\hline$\rho_{s}$ & density of solid \\
\hline$\sigma$ & stress \\
\hline$\varepsilon$ & strain \\
\hline$e$ & volumetric strain \\
\hline$u$ & displacement \\
\hline$E$ & Young's modulus \\
\hline$G$ & Shear modulus \\
\hline$K$ & modulus \\
\hline
\end{tabular}




\begin{tabular}{|c|c|}
\hline$v$ & Poisson's ratio \\
\hline$k_{r p}$ & is the relative permeability of phase $p$ \\
\hline$S_{p}$ & saturation of phase $p$ \\
\hline$b_{p}^{m}$ & molar density of phase $p$ \\
\hline$\mu_{p}$ & viscosity of phase $p$ \\
\hline$P_{c p}$ & capillary pressure for phase $p$ \\
\hline$\rho_{p}$ & mass density of phase $p$ \\
\hline$D$ & cell center depth \\
\hline$T_{n i}$ & transmissibility between cells $\mathrm{n}$ and $\mathrm{i}$. \\
\hline$\lambda_{m}$ & mobility of phase m \\
\hline$c_{r}$ & isothermal rock compressibility \\
\hline$c_{b}$ & bulk compressibility \\
\hline $\mathrm{g}$ & acceleration due to gravity \\
\hline $\mathrm{k}$ & absolute permeability \\
\hline$N_{I}$ & stock tank volume of component $\mathrm{i}$ \\
\hline$q_{i}$ & injection rate for phase $\mathrm{i}$ \\
\hline$U_{i}$ & fluid phase velocity \\
\hline$\alpha$ & poroelastic constant \\
\hline$\delta_{i j}$ & Kronecker's delta \\
\hline$\lambda$ & Lame's constant \\
\hline $\mathrm{x}, \mathrm{y}, \mathrm{z}$ & distance \\
\hline$\tau$ & time of flight \\
\hline
\end{tabular}


$Q \quad$ volumetric flux 


\section{REFERENCES}

1. Datta-Gupta, A., and King, M.J.: Streamline Simulation: Theory and Practice, SPE, Richardson, Texas (2007).

2. Kumar, S.: "Phase and Component Streamline Tracing Improves Understanding of Reservoir Dynamics,” MS Thesis, Texas A\&M U., College Station, Texas, 2008.

3. ECLIPSE® Reference Manual, 2007.1, Schlumberger Information Solutions.

4. ECLIPSE® Technical Description, 2007.1, Schlumberger Information Solutions.

5. Chhajlani, R., Zheng, Z., Mayfield, D., and MacArthur, B.: "Utilization of Geomechanics for Medusa Field Development, Deepwater Gulf of Mexico,” paper SPE 77779 presented at the 2002 SPE Annual Technical Conference and Exhibition, San Antonio, Texas, 29 September -2 October.

6. Giraldo, L.A., Chen, H-Y., and Teufel, L.W.: "Field Case Study of Geomechanical Impact of Pressure Depletion in the Low-Permeability Cupiagua GasCondensate Reservoir," paper SPE 60297 presented at the 2000 SPE Rocky Mountain Regional/Low Permeability Reservoirs Symposium, Denver, 12-15 March.

7. Kristiansen, T.G.: "Geomechanical Characterization of the Overburden above the Compacting Chalk Reservoir at Valhall," paper SPE 47348 presented at the 1998 SPE/ISRM Eurock, Trondheim, Norway, 8-10 July.

8. Heffer, K.J., Koutsabeloulis, N.C., and Wong, S.K.: "Coupled Geomechanical, Thermal and Fluid Flow Modeling as an Aid to Improving Waterflood Sweep Efficiency," paper SPE 28082 presented at the 1994 Eurock SPE/ISRM Rock Mechanics Petroleum Engineering Conference, Delft, Netherlands, 29-31 August. 
9. Chin, L.Y., Raghavan, R., and Thomas, L.K.: "Fully-Coupled Geomechanics and Fluid-Flow Analysis of Wells with Stress-Dependent Permeability," paper SPE 48857 presented at the 1998 SPE International Conference and Exhibition, Beijing, 2-6 November.

10. Giordano, R., M., Jayanti, S., Chopra, A., Yuan, H., Asakawa, K., Suleimani, A., Gheithy, A., and Edwards, C. : “A Streamline Based Reservoir Management Workflow to Maximize Oil Recovery," paper SPE 111143 presented at the 2007 SPE/EAGE Reservoir Characterization and Simulation Conference, Abu Dhabi, 28-31 October.

11. Baker, R.O., Kuppe F., Chugh, S., Bora, R., Stojanovic, S., and Batycky, R.:

"Full-Field Modeling Using Streamline-Based Simulation: Four Case Studies," paper SPE 66405 presented at the 2001 SPE Reservoir Simulation Symposium, Houston, 11-14 February.

12. Park, C., Kang, J.M., Jung, Y., and Kim, S.: "Streamline-Based Simulation to Investigate Interwell Connectivity and Tracer Transport in 3D Discrete Fractured Reservoir," paper SPE 100216 presented at the 2006 SPE Europec/EAGE Annual Conference and Exhibition, Vienna, 12-15 June.

13. Naguib, M., Sikaiti, S., Balushi, H. , Barrio, C., Mahrooqi, S., and Batycky, R.: "Results of Proactively Managing a Heavy-Oil Waterflood in South Oman Using Streamline-Based Simulation," paper SPE 101195 presented at the 2006 SPE Asia Pacific Oil \& Gas Conference and Exhibition, Adelaide, Australia, 11-13 September.

14. Stone, T., Bowen, G., Papanastasiou, P., and Fuller, J.: "Fully Coupled Geomechanics in a Commercial Reservoir Simulator," paper SPE 65107 presented at the 2000 SPE European Petroleum Conference, Paris, 24-25 October. 
15. Stone, T.W., Xian, C., Fand, Z., Manalac, E., Marsden, R., and Fuller, J.: “Coupled Geomechanical Simulation of Stress Dependent Reservoirs,” paper SPE 79697 presented at the 2003 SPE Reservoir Simulation Symposium, Houston, 3-5 February.

16. Jimenez, E.: "The Impact of Grid Geometry on Displacement Calculations," MS Thesis, Texas A\&M U., College Station, Texas, August 2004.

17. Pollock, D.W., "Semi-analytical Computation of Path Lines for Finite Difference Models" Ground Water, (November-December 1988) 26, 6.

18. Datta-Gupta A. and King M.J.: "A Semianalytic Approach to Tracer Flow Modeling in Heterogeneous Permeable Media," Adv. in Water Resources (1995), 18, No. 1,9 .

19. Chen, H.Y. and Teufel, L.W., and Lee, R.L.: "Coupled Fluid Flow and Geomechanics in Reservoir Study-I. Theory and Governing Equations," paper SPE 30752 presented at the 1995 SPE Annual Technical Conference and Exhibition, Dallas, 22-25 October.

20. Biot, M.A.: "Theory of Elasticity and Consolidation for a Porous Anisotropic Solid," Journal of Applied Physics (1955) 26, 182-185.

21. Biot, M.A.: "General Theory of Three-Dimensional Consolidation," Journal of Applied Physics (1941) 12, 155-164.

22. Biot, M.A.: "General Solutions of the Equations of Elasticity and Consolidation for a Porous Material," Journal of Applied Mechanics (1956) 27, 91-96.

23. Biot, M.A.: "Thermoelasticity and Irreversible Thermodynamics," Journal of Applied Physics (1956) 27, 240-253.

24. Biot, M.A. and Willis, D.: "The Elastic Coefficients of the Theory of 
Consolidation," Journal of Applied Mechanics (1957) 24, 594-601.

25. Biot, M.A.: "Mechanics of Deformation and Acoustic Propagation in Porous Media," Journal of Applied Physics (1962) 33, 1482-1498.

26. Biot, M.A.: "Nonlinear and Semilinear Rheology of Porous Solids," J. Geophys. Res. (1973) 78, 49244937.

27. Geertsma, J: "The Effect of Fluid Pressure Decline on Volumetric Changes of Porous Rock," Trans. AIME (1957) 210, 331-340.

28. Brown, R.J.S. and Korringa, J. "On the Dependence of the Elastic Properties of a Porous Rock on the Compressibility of the Pore Fluid," Geophysics (1975) 40, 608-616.

29. Terzaghi, K.: Theoretical Soil Mechanics, Wiley, New York (1943).

30. Nur, A. and Byerlee, J.D.: "An Exact effective Stress Law for Elastic Deformation of Rock with Fluids," J. Geophys. Res. (1971) 76, 6414-6419.

31. Verrujit, A.: "Elastic Storage in Aquifers," Flow Through Porous Media, R.J.M De Wiest (ed.), Academic, San Diego, California (1969) 331-376.

32. Minkoff, S.E., Stone, C.M., Bryant, S., Peszynska, M. and Wheeler, M.F.: "Coupled Fluid Flow and Geomechanical Deformation Modeling," Journal of Petroleum Science and Engineering (2003) 38, 37-56.

33. Settari, A. and Mourits, F.M.: "A Coupled Reservoir and Geomechanical Simulation System," SPEJ (September 1998) 219. 


\section{APPENDIX \\ CHANGES INCORPORATED IN THE DATA FILE FOR CONVENTIONAL RUN TO CARRY OUT COUPLED GEOMECHANICS SIMULATION RUN}

For simulation studies, ECLIPSE-300® simulator with Cartesian grid and corner point geometry was used:

- In the RUNSPEC section, geomechanics option was activated by incorporating "GEOMECH" keyword. "GEODIMS" keyword was also incorporated to tell the simulator about size of the permeability modifier table.

- In GRID section, in addition to grid property and geometry keywords; keywords for the following geomechanics properties were used: Young's modulus, Poisson's ratio, Rock density (all three for Rock/Stress Balance) and Biot's constant (for Rock /Fluid interaction). After the initialization of the simulation study, if it is found that the reservoir pore volume for coupled geomechanics run does not matches with the reservoir pore volume for conventional simulation run than the value of Young's Modulus needs to be changed till the match in pore volume for both the simulation is obtained.

- In PVT section, permeability modifier table is incorporated through "PERMSTAB" keyword. If plasticity option is to be used then geomechanics yield function parameter keyword (which gives information about rock cohesive strength, failure model, angle of friction and type of hardening law to be used) needs to be included. 
- In SOLUTION section, stress boundary conditions are incorporated through "GMTRABC" keyword and displacement boundary conditions are incorporated though "GMDISBC".

- For a field case study, the geo-cellular grid is populated with rock mechanical properties obtained through interpretation of sonic well logs and core study. 


\section{VITA}

Name:

Address

Email Address:

Education:

Experience:
Prannay Parihar

c/o Dr. Akhil Datta-Gupta

3116 TAMU, RICH-702

Dept. of Petroleum Engineering

Texas A\&M University, College Station

TX-77843

prannay.parihar@gmail.com

B.S., Petroleum Engineering

Indian School of Mines, Dhanbad, India, 2004

M.S., Petroleum Engineering

Texas A\&M University, 2008

Petroleum Engineer, Reliance Industries Ltd., India. 20042006

Summer Intern, Chevron NAEP, Houston, (Summer) 2007 